\title{
Multifilar Hemispherical Helical Antennas
}

\author{
Jeffrey R. Clark \\ Thesis submitted to the faculty of the \\ Virginia Polytechnic Institute and State University \\ in partial fulfillment of the requirements of the degree of \\ Master of Science \\ in \\ Electrical Engineering
}

Ahmad Safaai-Jazi, Chair

Ioannis M. Besieris

William A. Davis

October 31, 2003

Blacksburg, Virginia

Keywords: Helical Antennas, Quadifilar Helix, Multifilar Helices, NEC-4

Copyright 2003, Jeffrey R. Clark 


\title{
Multifilar Hemispherical Helical Antennas
}

\author{
Jeffrey R. Clark
}

ABSTRACT

Helices are broadband antennas that provide moderate gain, largely real input impedance and circular polarization when operating in the axial mode regime. A modified form of the helix, the spherical helix, has been shown to yield similar polarization and gain characteristics over a narrow bandwidth but a much larger beamwidth. This investigation examines multifilar hemispherical helices and produces two specific designs with some desirable radiation characteristics. The side-fed quadrifilar helix and the top-fed bifilar helix are the new hemispherical designs which are studied in detail both numerically and experimentally.

The Numerical Electromagnetics Code 4 is used to analyze the radiation characteristics of the proposed multifilar hemispherical helices. Directivity, E- and Hplane radiation patterns, axial ratio and input impedance of a few example designs are calculated. Measurements, taken in the anechoic chamber at the Virginia Tech antenna range, generally confirmed the simulation results. It is shown that the bifilar hemispherical helix provides a flat gain curve over the measured bandwidth $(\sim 14 \%)$ and generally elliptical polarization with near circular polarization in limited case. 


\section{Acknowledgements}

I would like to thank my advisor, Dr. Ahmad Safaai-Jazi, for all his help and patience throughout my work on this thesis, as well as for providing a topic of research and guiding me in studying new applications. My thanks also go to Drs. Ioannis M. Besieris and William A. Davis for their service on my committee.

Also deserving a note of gratitude are Taeyoung Yang for his patient, outstanding assistance in making pattern measurements, and Dr. Ahmed Salem for his time and help in making input impedance measurements. 


\section{Table of Contents}

Chapter 1. Introduction.................................................................................................. 1

Chapter 2. The Helical Antenna................................................................................... 3

2.1 Helix Radiation Modes............................................................................ 5

2.1.1 Normal Mode Analysis................................................................... 6

2.1.2 Axial Mode Analysis....................................................................... 8

2.2 Numerical Analysis of the Helix.................................................................... 12

2.3 Modified Forms of the Helix.................................................................. 15

2.3.1 The Quadrifilar Helical Antenna................................................... 16

2.3.2 The Bifilar Helical Antenna........................................................ 18

Chapter 3. The Spherical Helix................................................................................... 20

3.1 Radiation Characteristics of the Spherical Helix............................................ 22

3.1.1 The Axial and Axial-null Modes..................................................... 22

3.2 The Truncated Spherical Helix.................................................................... 24

Chapter 4. The Multifilar Hemispherical Helix...................................................... 26

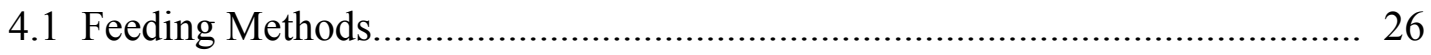

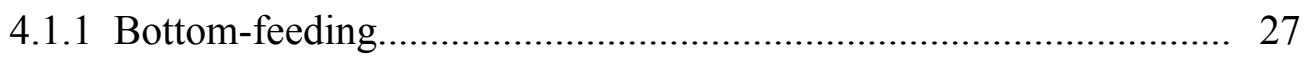

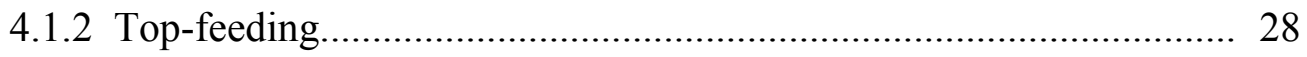

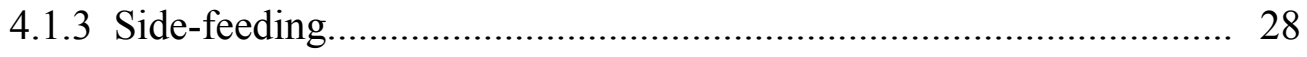

4.2 Multifilar Hemispherical Helices.............................................................. 29

4.3 Simulation of Multifilar Hemispherical Helices Using NEC-4 ....................... 30

4.4 The Top-fed Bifilar Hemispherical Helix....................................................... 31 
4.5 The Side-fed Quadrifilar Hemispherical Helix........................................... 35

Chapter 5. Prototype Construction and Measurement.................................... 40

5.1 Construction Procedures...................................................................... 40

5.2 Pattern Measurement Setup................................................................ 42

5.3 Voltage Standing Wave Ratio Measurements.......................................... 43

5.3.1 Adjustment of the Simulated Diameter of the Bifilar Hemisphere.... 45

5.4 Gain Measurement for the Bifilar Hemisphere ......................................... 46

5.4.1 Pattern Measurement........................................................... 48

5.5 Measurements for the Quadrifilar Hemisphere........................................ 50

5.5.1 Pattern Measurement............................................................ 51

Chapter 6. Conclusions and Suggestions for Further Work............................... 54

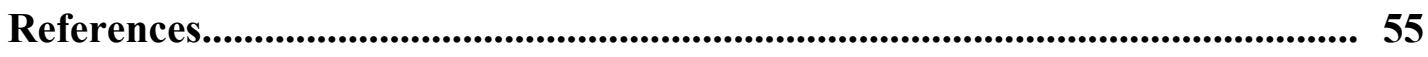

Appendix A. Pattern Data for the Bifilar Hemispherical Helix......................... 57

Appendix B. Pattern Data for the Quadrifilar Hemispherical Helix............... 80

Appendix C. Input Impedance Plots.................................................................. 87

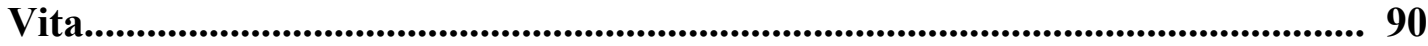




\section{List of Figures and Tables}

Table 2.1 Helical geometry definitions............................................................ 3

Figure 2.1 A helical antenna...................................................................... 4

Figure 2.2 Geometrical relationships for a helix............................................ 5

Figure 2.3 Computed patterns for a helix in normal and axial modes................... 6

Figure 2.4 An approximate representation of the normal mode helix.................. 7

Figure 2.5 Analytical patterns for a normal and an axial mode helix................... 11

Figure 2.6 Computed and measured patterns for the helix compared................... 12

Figure 2.7 Axial ratio and standing wave ratio measurements for 6-turn helix...... 13

Figure 2.8 Axial ratio and standing wave ratio simulated results for 6-turn helix.. 14

Figure 2.9 Parameters for modifying the helix............................................... 15

Figure 2.10 A quarter-turn quadrifilar helical (volute) antenna.......................... 17

Figure 2.11 Computed patterns for a half-turn volute antenna............................ 17

Figure 2.12 Measured patterns for a 5-turn-per-element quadrifilar helix............. 18

Figure 2.13 A bifilar helical antenna with 3 turns per element............................ 19

Figure 2.14 Computed patterns for a 3-turn-per-element bifilar helix.................. 19

Figure 3.1 An 8-turn spherical helical antenna over ground plane...................... 20

Figure 3.2 A 5-turn truncated spherical helix................................................... 23

Figure 3.3 Computed patterns for a spherical helix in axial and axial-null modes.. 25

Figure 4.1 A side-fed 4-turn hemispherical helix with ground plane.................... 27

Figure 4.2 A bottom-fed hemispherical helix.................................................. 28

Figure 4.3 A top-fed hemispherical helix....................................................... 29

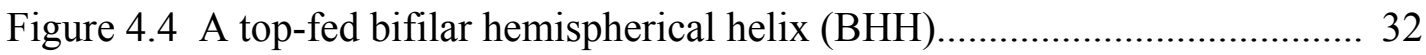

Figure 4.5 Currents on bifilar hemispherical helix........................................ 32

Figure 4.6 Computed E-plane pattern for bifilar hemispherical helix.................. 33

Figure 4.7 Computed standing wave ratio for the bifilar hemispherical helix...... 34

Figure 4.8 Computed H-plane axial ratio and phase difference for a BHH.......... 35

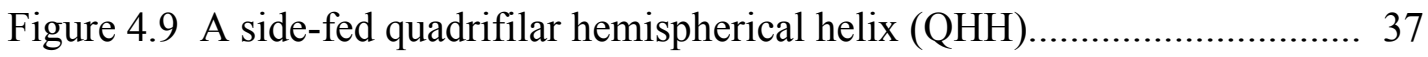

Figure 4.10 Computed results for the standing wave ratio of a QHH................. 37

Figure 4.11 Computed pattern for a QHH.................................................. 38 
Figure 4.12 Computed axial ratio and phase difference for a QHH................... 39

Figure 5.1 Prototype BHH in anechoic chamber.............................................. 41

Figure 5.2 Prototype QHH in anechoic chamber.......................................... 42

Figure 5.3 Measurement setup in the anechoic chamber.................................... 43

Figure 5.4 Diagram of setup for standing wave ratio measurement.................... 44

Figure 5.5 Data comparison for standing wave ratio of BHH.......................... 45

Figure 5.6 Data comparison for the gain of BHH.......................................... 46

Figure 5.7 Data comparison for the gain in the E-plane of BHH....................... 47

Figure 5.8 Data comparison for the gain in the H-plane of BHH........................ 47

Figure 5.9 Measured E-plane pattern for BHH............................................ 48

Figure 5.10 Comparison of axial ratio and phase difference data for BHH......... 50

Figure 5.11 Data comparison for standing wave ratio of QHH......................... 51

Figure 5.12 Comparison of axial ratio and phase difference data for QHH......... 52

Figure 5.13 Measured pattern for QHH....................................................... 53 


\section{Chapter 1. Introduction}

Wire antennas are ubiquitous and highly recognizable, being used for a vast range of communications applications. Although some simple wire antennas, such as dipoles and monopoles, may be modeled analytically, the vast majority of configurations are of such a complexity that requires numerical calculation of their properties. As computing power has become more abundant and inexpensive, however, the ability to simulate wire antennas of arbitrary design quickly and cheaply has become possible.

A wire antenna may range from a resonant antenna, where the dimensions are small with respect to wavelength and the antenna supports standing waves, to a traveling wave antenna, where the dimensions are large with respect to wavelength and standing waves are not supported. Arrays may also be formed using multiple antenna elements.

Many exotic types of antennas have been developed for the purpose of improving or increasing bandwidth, gain, certain polarization qualities, beamwidth or other characteristics. One such example is the helical antenna, or helix, which under the right circumstances may provide nearly real input impedance, moderate gain and circular polarization over a significant beamwidth and bandwidth. Other examples include vee dipoles, spiral antennas and arrays of various kinds of antennas in numerous arrangements.

The method of moments is a standard tool for computing antenna characteristics at a given frequency and has been found to produce generally reliable results, although input impedance calculations sometimes lack accuracy. It provides a powerful method 
for investigating or optimizing an antenna without the need of constructing a prototype model for each variation.

In this investigation, the method of moments based code, Numerical Electromagnetics Code 4 (NEC-4), was employed to simulate hemispherical helical wire antennas with multiple arms. NEC-4 uses a hybrid solution of electric and magnetic field integral equations (EIFE and MFIE) to simulate the performance of an antenna. The EIFE is used primarily for thin wires, whereas MFIE is used for surfaces. These equations are solved numerically by Galerkin's method with current expansions in terms of sinusoids [1].

The usefulness of bifilar, trifilar and quadrifilar hemispherical helices was examined under various conditions, and the two most interesting designs, the symmetric bifilar and quadrifilar hemispheres were constructed in prototype and measured at the Virginia Tech indoor antenna range. It is hoped that the characteristics of these antennas will find some application, possibly in Global Positioning System (GPS) or other communications applications, or will provide a basis for further modification or optimization. 


\section{Chapter 2. The Helical Antenna}

The helical antenna (helix) is a traveling-wave, broadband antenna which was introduced and investigated by Kraus [2]. Geometrically, the simple helix can be represented in terms of three simple and easily measured spatial characteristics: height, diameter and spacing between turns. Other useful parameters, such as the pitch angle, length of a turn, number of turns and circumference maybe found easily using these values. The nomenclature for the geometry of the helix is shown in Table 2.1; Figure 2.1 depicts a 3-turn helix with most of the relevant parameters shown.

It is heuristically useful to look at a single turn which has been uncoiled to visualize some of these relationships, as shown in Figure 2.2. The turn length, $\mathrm{L}$, is straightened to reveal a right triangle scenario with the turn spacing, S, and the helical circumference, $\mathrm{C}$.

Table 2.1. Definitions for the geometry of the helix.

\begin{tabular}{|l|l|}
\hline h & height \\
\hline d & helical diameter \\
\hline D & conductor diameter \\
\hline S & spacing between turns \\
\hline a & pitch angle \\
\hline L & length of a turn \\
\hline N & number of turns \\
\hline C & helical circumference \\
\hline
\end{tabular}




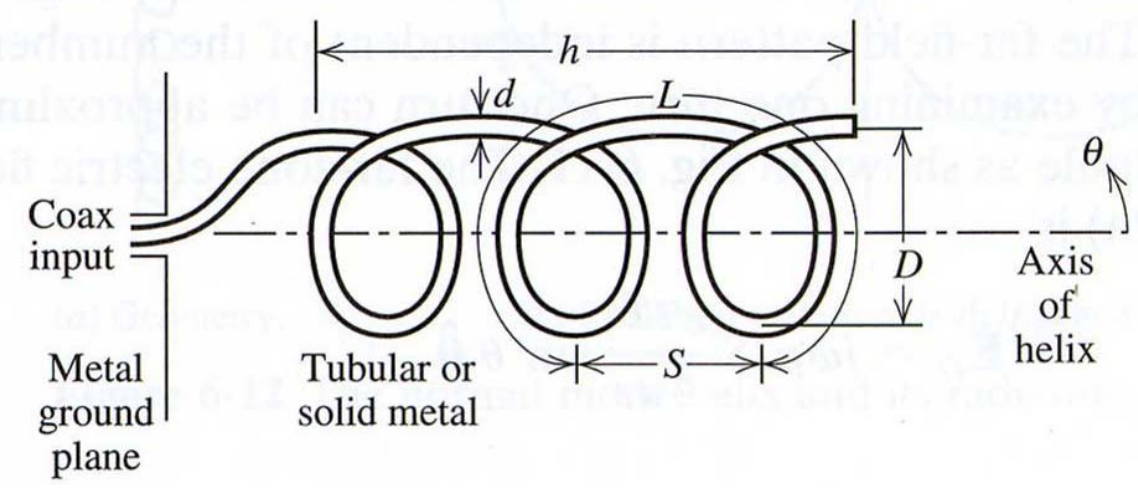

Figure 2.1: A three turn helical antenna with a ground plane and coaxial input.

Some relevant relations among the parameters of the helix are summarized below. These include the proportional relationship of the circumference (C) and diameter (D), since the helix has a circular cross section, and the number of turns $(\mathrm{N})$ in terms of the height (h) and turn spacing (S), since each turn corresponds to a given distance on the helical axis and the height of the helix is the total distance along the axis. The relationships for the length of one turn (L) and the pitch angle $(\alpha)$ may be found using simple right triangle trigonometry.

$$
\begin{aligned}
& \mathrm{C}=\pi \mathrm{D} \\
& \mathrm{L}=\sqrt{S^{2}+C^{2}} \\
& \alpha=\arctan \left(\frac{S}{C}\right) \\
& \mathrm{N}=\frac{h}{S}
\end{aligned}
$$




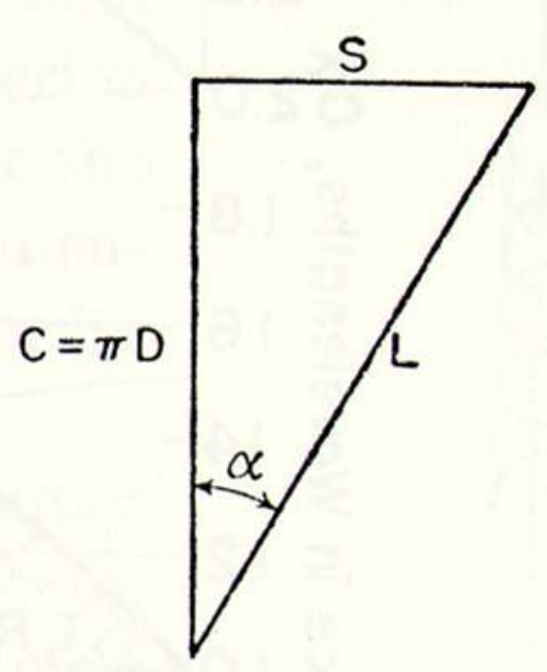

Figure 2.2: A single turn of the helix is straightened to reveal the relationships among the geometrical parameters.

\subsection{Helix Radiation Modes}

The helix has two operating regimes: the normal mode and the axial mode [2]. The normal mode dominates when the wavelength of operation is larger than the diameter of the helix, whereas the axial mode dominates when the wavelength is smaller than the diameter. Of interest is the axial mode in which radiation is most intense along the axis of the helix and which produces a moderate gain and circular polarization for certain designs. It has been found that helices of several turns have acceptable characteristics when the electrical size (circumference) is between $3 / 4 \lambda$ and $4 / 3 \lambda$, yielding a bandwidth of $56 \%$. Figure 2.3 shows pattern plots for an axial mode and a normal mode helix obtained using the method of moments code NEC-4. 

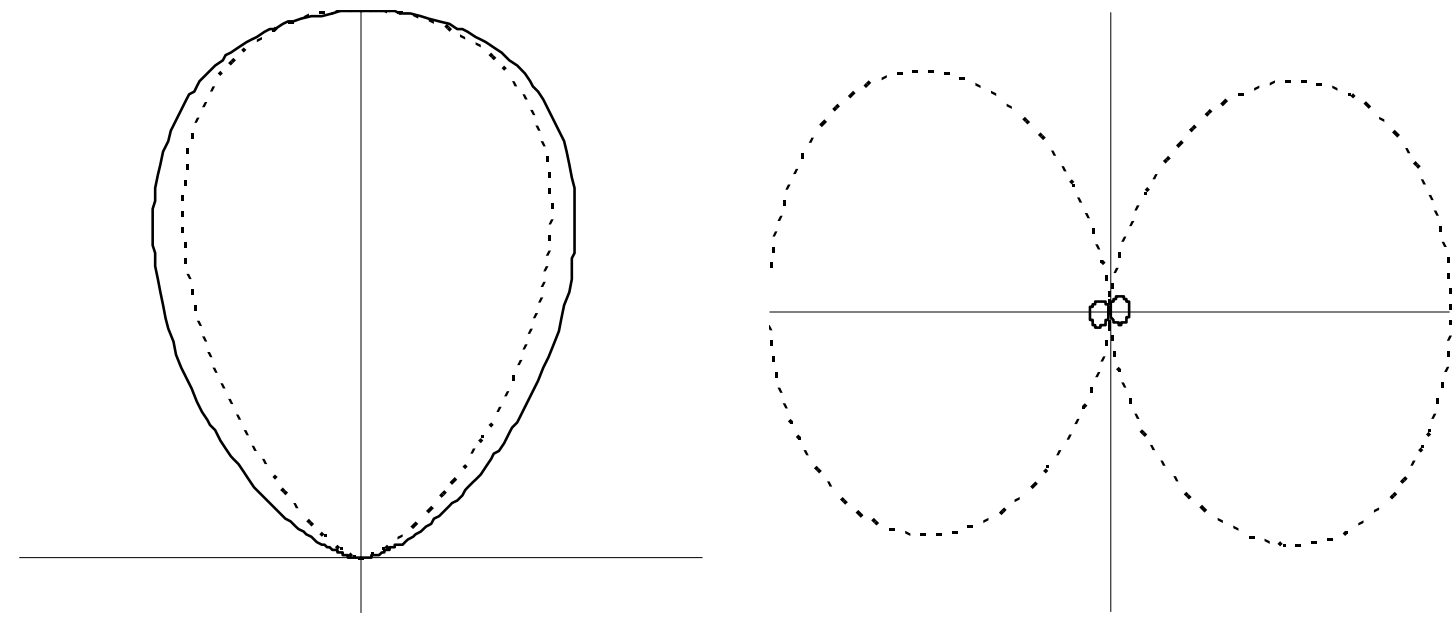

Figure 2.3: An axial mode pattern (left) and a normal mode pattern (right) for a 6-turn helical antenna. These patterns were obtained through simulation using NEC-4. Shown are the $\theta$ (dashed) and $\varphi$ (solid) components of the electric field.

\subsubsection{Normal Mode Analysis}

Although analytical models of all but very simple antennas are difficult, if not impossible, to produce, the normal mode helix may be modeled as a small loop and small dipole, as shown in Figure 2.4. This, then, requires that the normal mode helix be small with respect to the wavelength of operation in terms of length and diameter. That is, $\mathrm{L}$ $<<\lambda$ and $\mathrm{D}<<\lambda$. This analysis is independent of the number of turns and may proceed by examining a single turn [3]. The resulting far-field pattern for the normal mode helix is the vector sum of the patterns resulting from the loop and the dipole individually. These are shown below with S being the spacing between turns (related to dipole length) and $\mathrm{D}^{2} \pi / 4$ is the cross sectional area of the helix (related to the area of the loop).

$$
\mathbf{E}_{\mathrm{D}}=\mathrm{j} \omega \mu \mathrm{IS} \frac{e^{j \beta r}}{4 \pi r} \sin \theta \hat{\boldsymbol{\theta}}
$$




$$
\mathbf{E}_{\mathrm{L}}=\eta \beta^{2} \frac{\pi}{4} \mathrm{D}^{2} \mathrm{I} \frac{e^{j \beta r}}{4 \pi r} \sin \theta \hat{\boldsymbol{\varphi}}
$$

The pattern for the normal mode helix, then, is given in (2-7) for both the $\theta$ and $\varphi$ components of the field. This result is plotted in Figure 2.5.

$$
\mathrm{F}(\theta)=\sin \theta
$$
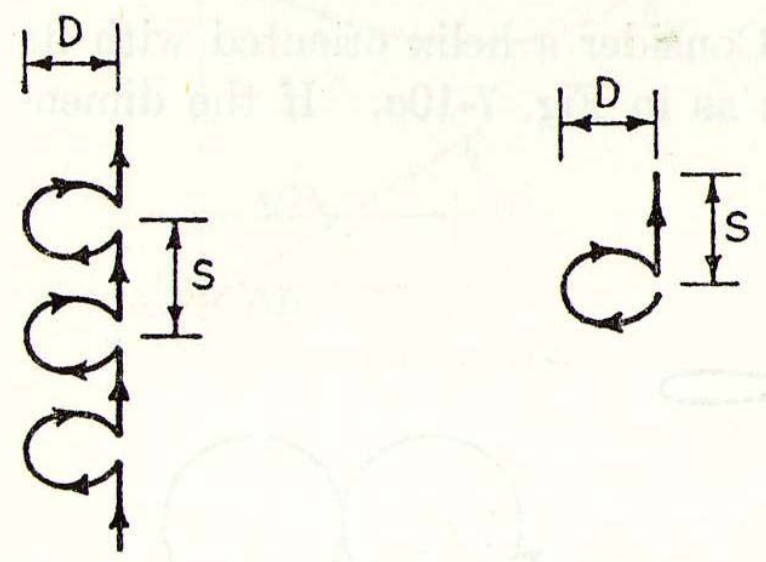

Figure 2.4: A normal mode helical antenna may be modeled as a set of dipoles and loops (left) and then reduced to a single loop and dipole due to the small electrical size.

It is noted from equations (2-5) and (2-6) that these two components are $90^{\circ}$ out of phase, and therefore can produce circular polarization when equal in magnitude. The ratio of their magnitudes reduces to:

$$
\frac{\left|E_{\theta}\right|}{\left|E_{\phi}\right|}=\frac{2 S \lambda}{\pi^{2} D^{2}}
$$


Setting (2-8) equal to 1 produces yields the condition for circular polarization. Using some of the geometrical relationships for the helix (equations (2-1) to (2-4)), the resulting condition reduces to the circular polarization condition in (2-9) [3].

$$
\alpha_{C P}=\sin ^{-1}\left(\frac{-1+\sqrt{1+(L / \lambda)^{2}}}{L / \lambda}\right)
$$

\subsubsection{Axial Mode Analysis}

The axial mode helix can be approximated by modeling each turn as a simpler element in an array. Array theory may then be invoked and the radiation pattern found as the product of an $\mathrm{N}$-element array ( $\mathrm{N}$-turn helix) pattern and the simplified element pattern.

Since the helix performs best for axial mode with normalized circumferences between $3 / 4 \lambda$ and $4 / 3 \lambda$, each turn has a length of approximately one wavelength. Since the helix is essentially a traveling-wave antenna, and assuming the current is nearly constant along the wire, then the currents on opposite sides of the turn are about $180^{\circ}$ out of phase. This means that these currents are nearly equivalent in direction, resulting in constructive interference of the radiation along the axis of the helix. This behavior is similar to the one-wavelength loop [3], and is easily seen when the pitch angle of the helix goes to zero, causing the antenna to degenerate into a loop. The element pattern of the array, then, is approximately $\cos \theta$, which is the pattern of the one-wavelength circular loop.

Invoking array theory, the array factor, $\operatorname{AR}(\theta)$, for an equally spaced $\mathrm{N}$ element (N-turn) linear array (helix) is expressed as: 


$$
\mathrm{F}(\theta)=\frac{\sin (N \psi / 2)}{N \sin (\psi / 2)}
$$

where:

$$
\psi=\beta S \cos \theta+\alpha_{h}
$$

with $\mathrm{S}$ being the element spacing, $\beta$ the wave number, and $\alpha_{\mathrm{h}}$ the inter-element phase shift. The total pattern is then obtained as:

$$
\mathrm{F}(\theta)=\mathrm{K} \cos \theta \frac{\sin (N \psi / 2)}{N \sin (\psi / 2)}
$$

where $\mathrm{K}$ is a normalization constant. It has been determined that the inter-element phase shift, $\alpha_{\mathrm{h}}$ is that of a Hansen-Woodyard array and is given as:

$$
\alpha_{\mathrm{h}}=-\left(\beta \mathrm{S}+2 \pi+\frac{\pi}{N}\right)
$$

The normalization factor, $\mathrm{K}$, can be found simply using the determined beam maximum along the axis of the helix (array), or $\theta=0^{0}$. Then:

$$
\begin{aligned}
& \psi=\beta \mathrm{S}+\alpha_{\mathrm{h}}=-2 \pi-\frac{\pi}{N} \\
& \mathrm{~F}(0)=1=\mathrm{K} \frac{\sin (-N \pi-\pi / 2)}{N \sin (-\pi-\pi / 2 N)}
\end{aligned}
$$


Through the use of trigonometric identities:

$$
K=(-1)^{N+1} N \sin (\pi / 2 N)
$$

The total pattern is then:

$$
\mathrm{F}(\theta)=(-1)^{\mathrm{N}+1} \sin \left(\frac{\pi}{2 N}\right) \cos \theta \frac{\sin (N \psi / 2)}{\sin (\psi / 2)}
$$

This result is an approximate analytical pattern for the helix based on array theory and is plotted in Figure 2.5. The inclusion of an infinite ground plane is also possible by including the image of the helix (array) below the ground plane.

Some empirical models for helix performance were produced by Kraus [2] and then later, more intensively, by King and Wong [4]. An empirical formula developed by King and Wong for the peak gain (G) is given in (2-18), where $\lambda_{\mathrm{p}}$ is the wavelength at peak gain.

$$
\mathrm{G}=8.3\left(\frac{\pi D}{\lambda_{p}}\right)^{\sqrt{N+2}-1}\left(\frac{N S}{\lambda_{p}}\right)^{0.8}\left(\frac{\tan 12.5^{\circ}}{\tan \alpha}\right)^{\sqrt{N} / 2}
$$

Kraus has shown that the axial ratio is approximately given by (2-19) for an N-turn helix operating in the axial mode [2]. This implies that the axial ratio approaches unity for a helix with a large number of turns. 


$$
\mathrm{AR}=\frac{2 N+1}{2 N}
$$

It is noteworthy that this is specific to the helix. In general, the axial ratio is defined by (2-20) [5]. Left-hand circular polarization is represented by a ' + ' sign and right-hand circular polarization is represented by a '-' sign. A completely general method for calculating the axial ratio, given the magnitude and phase of the orthogonal components of the field, is available as well [6].

$$
\mathrm{AR}= \pm \frac{E_{\text {major axis }}}{E_{\text {min or axis }}}
$$

An approximate formula for the input resistance of the helix is given by (2-21) [2].

$$
\mathrm{R}_{\mathrm{A}}=140 \frac{C}{\lambda} \Omega
$$
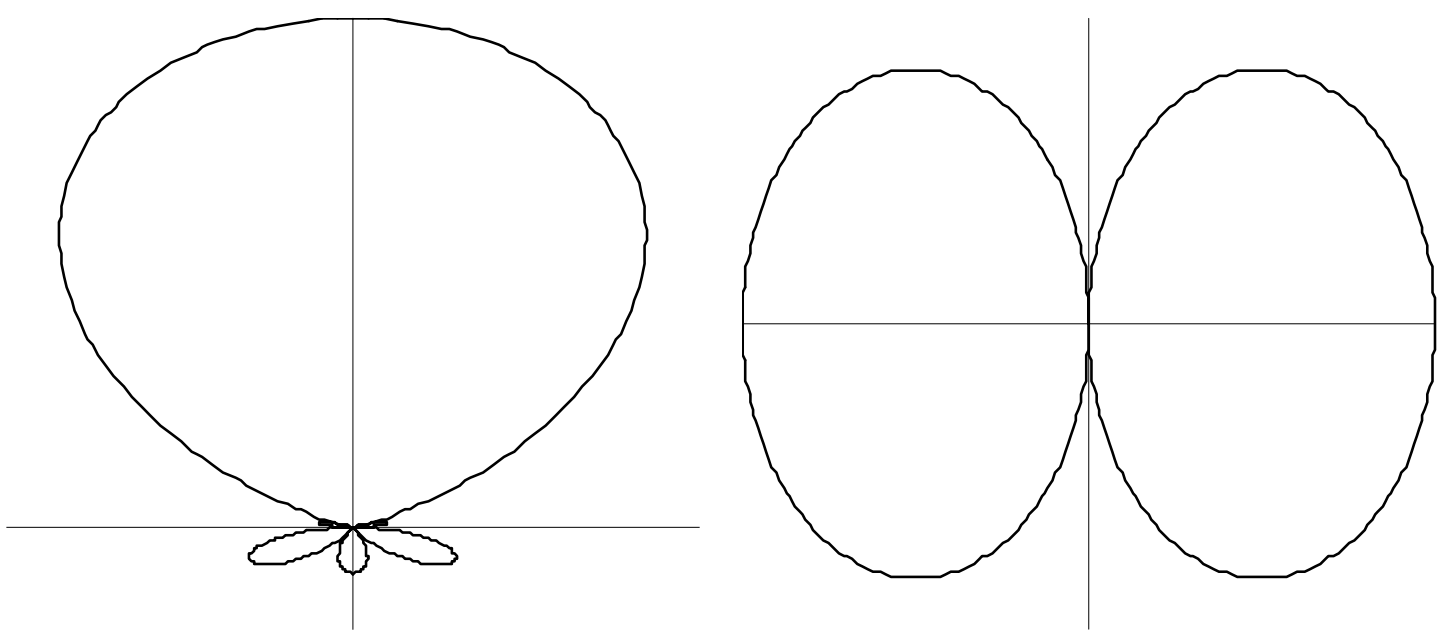

Figure 2.5: Analytical patterns for a 6 turn helix in axial mode (left) and normal mode (right). The axial mode helix has a normalized length of $1.6 \lambda$. 


\subsection{Numerical Analysis of the Helix}

Far-field patterns for a 6-turn helix with a pitch angle $(\alpha)$ of $14^{\circ}$ were produced using the method of moments software Numerical Electromagnetics Code 4 (NEC-4). A square ground plane with a side length of $0.8 \mathrm{~m}$ was used, and the connecting wire from the feed point (at the origin) to the base of the helix was set at an angle $\alpha$ from the ground plane. The helix was simulated with 120 segments and was simulated over the same normalized circumference range as used by Kraus [2]. The resulting patterns were generally in agreement with measured results, excepting some minor variations attributable to numerical error or deviations from ideal geometry in the measured helix. Also, Kraus does not describe the ground plane used, thus the parameters used by Cardoso were employed here [7]. The results are shown in Figure 2.6.

(a)
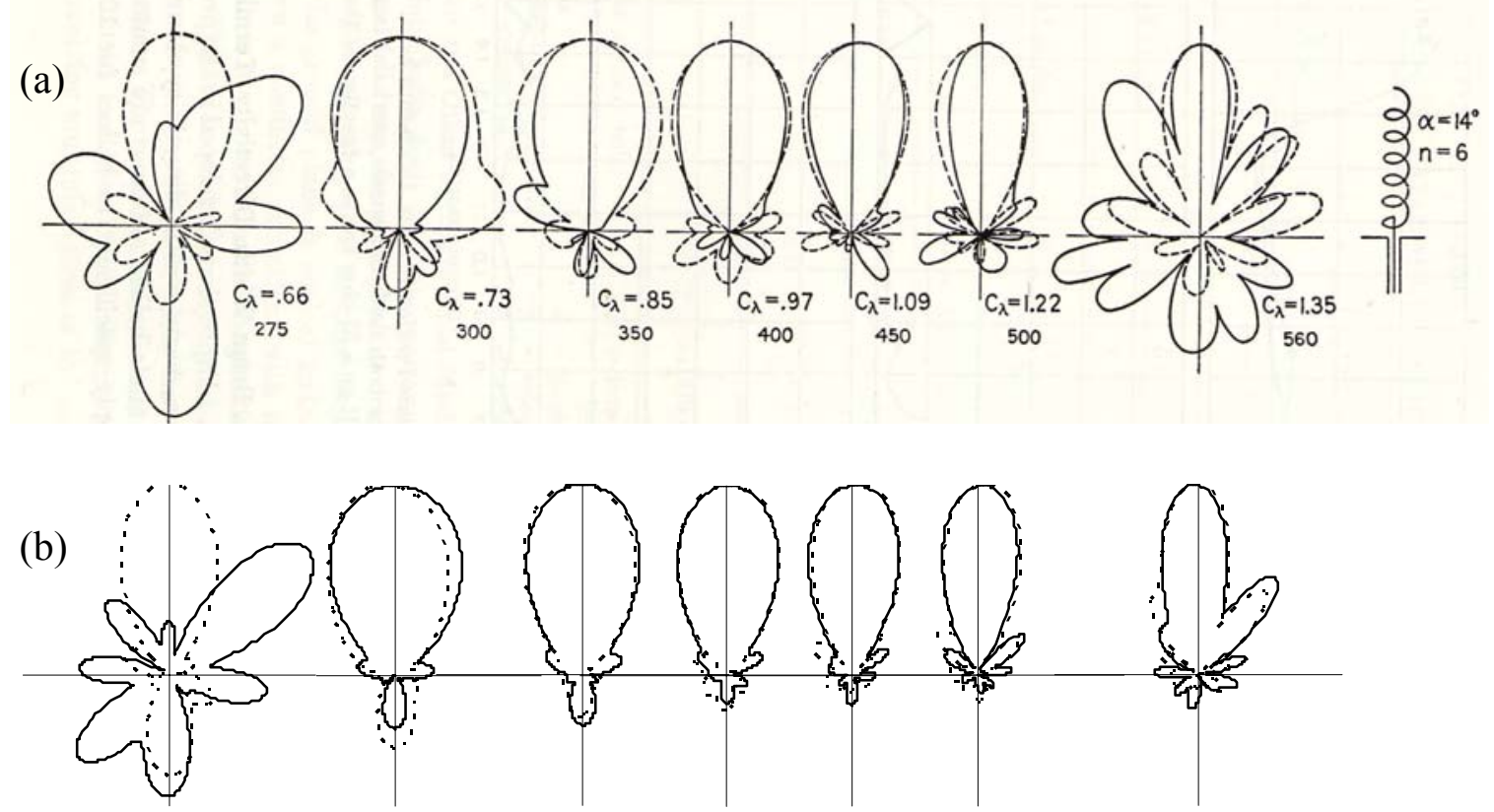

Figure 2.6: A comparison of measured results (a) and simulated results using NEC-4 (b) for a 6-turn helical antenna with a pitch angle of $14^{\circ}$. The normalized circumferences and frequencies correspond to a diameter of $0.66 \mathrm{~m}$. The solid curves correspond to the $\varphi$ component of the electric field and the dashed curves to the $\theta$ component. 
The axial ratio and voltage standing wave ratio (VSWR) obtained from NEC-4 for this antenna may also be compared to measured results. (In all cases in this investigation, the VSWR is in the form of a linear ratio rather than in decibels.) For both parameters, the simulated results follow the same general trends as those measured by Kraus. The greatest deviation occurs in the VSWR, where the simulated results yield slightly higher values over the middle to latter portion of the normalized circumference range, and very much higher values over the early portion of the range. The measured results of Kraus are shown in Figure 2.7, and the simulated results from NEC-4 are shown in Figure 2.8.

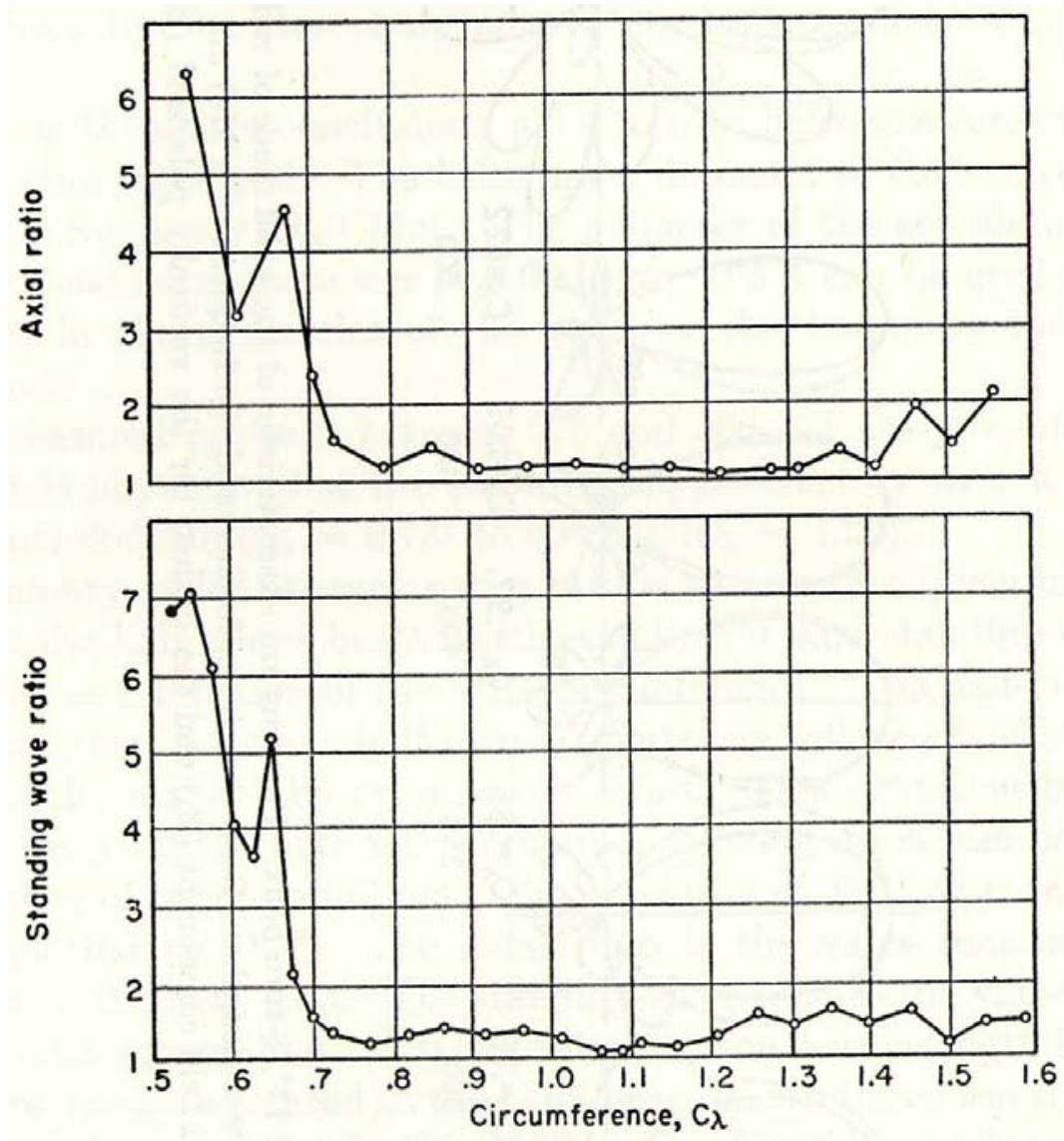

Figure 2.7: The measured values for axial ratio and VSWR for the 6-turn helix over a range of normalized circumferences. 

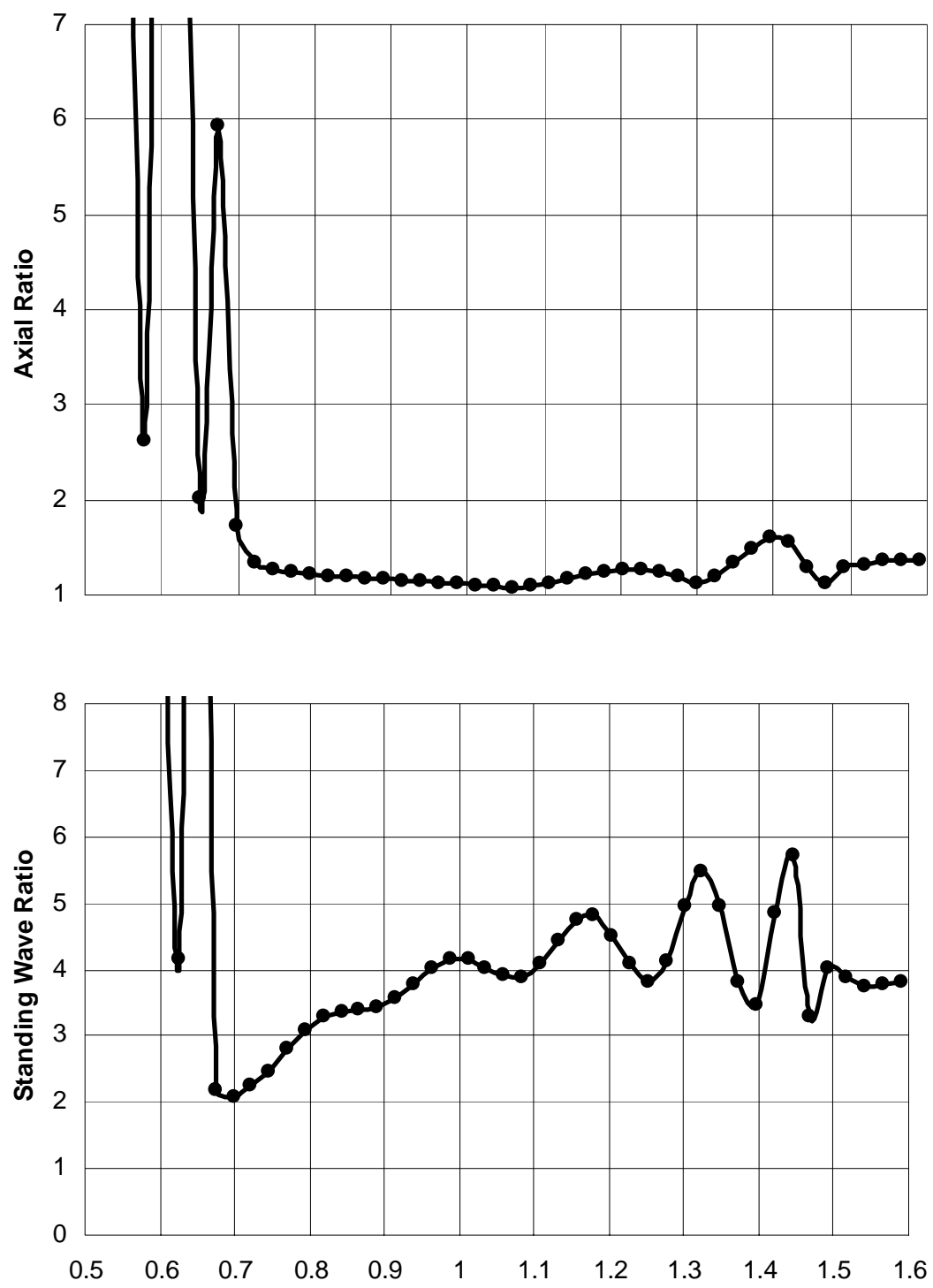

Figure 2.8: Simulated results for the axial ratio (top) and VSWR (bottom) of the 6-turn helix.

Although there are some deviations in the results obtained from NEC-4 with respect to the measured results, it is apparent from this example that NEC-4 is a useful numerical tool for obtaining a good estimate of the performance of an antenna over a range of frequencies. As noted earlier, however, the simulated results for the VSWR are suspect, as evidenced by the more significant divergence of the results seen upon 
comparison. This concern is noteworthy, as measurement becomes virtually the only method for accurate determination of the VSWR for a new antenna, thus complicating simulation and optimization.

\subsection{Modified Forms of the Helix}

The helix may be modified by varying certain geometrical parameters in an attempt to improve the characteristics of the antenna, as summarized in Figure 2.9. This may be done in various ways, including variation of the diameter (D), pitch angle $(\alpha)$ or spacing between turns (S). According to Kraus, this may be done by holding any one parameter constant and varying the other two. Several recent modifications to the helix have been attempted, including a nonlinearly tapered helix [8] and a spherical helix $[9,10]$.

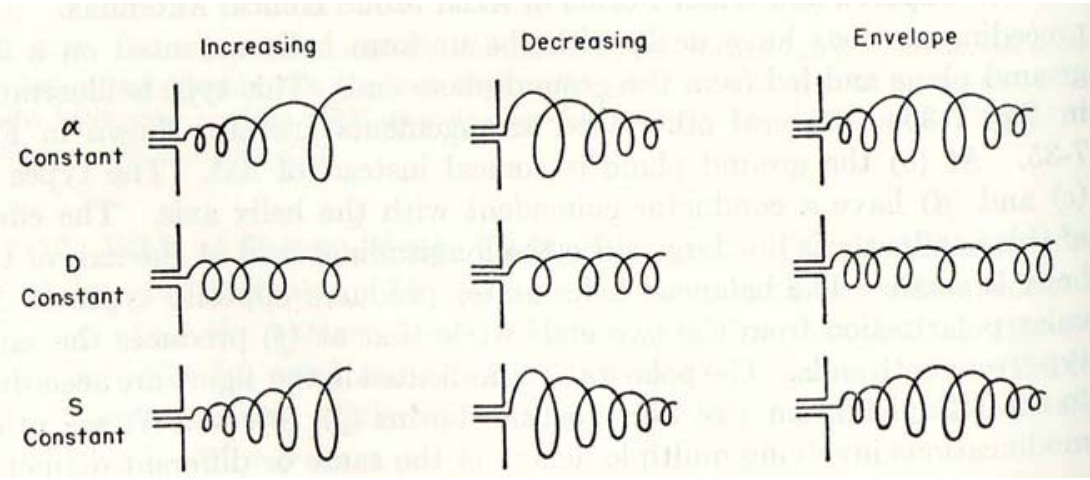

Figure 2.9: A summary of the geometrical modifications of the helical antenna.

Helical antennas composed of many coaxial helices are also widely used for satellite and mobile communications. Among these are the bifilar (two element) and, most prominently, quadrifilar (four element) helical antennas. An octifilar (eight element) helix has even been investigated [11]. It has been found that by feeding the 
elements with appropriate phase differences, desirable patterns with very pure circular polarization may be obtained. In the case of the bifilar helix, the two elements are fed in antiphase, while the quadrifilar helix has adjacent elements fed in phase quadrature.

\subsubsection{The Quadrifilar Helical Antenna}

One particular form of the quadrifilar version is the so-called resonant quadrifilar helix antenna, or volute antenna, which produces a broad circularly polarized endfire beam $[3,12]$. This antenna involves four half-wavelength windings which are fed at the open end and are shorted to the ground plane, as shown in Figure 2.10. These windings are in the range of quarter- to full-turn helices. A $90^{\circ}$ phase shifter is used to feed the orthogonal pairs of helices in quadrature. An example pattern is shown in Figure 2.11. The volute antenna is not a broadband device, as it produces a small bandwidth of only several percent [13]. This antenna finds an important application in the Global Positioning System (GPS), where the small size and circular polarization are critical features [14].

The quadrifilar helix may also be designed with many turns per element. This is a more general design than the volute antenna previously mentioned. This antenna has been found to be very broadband, with a bandwidth up to 5:1 [15]. Also, the quadrifilar helix may be designed to radiate a bifurcated, or conical, beam, as shown in Figure 2.12. It has been found that the axial ratio for this type of antenna is consistently between 3 and $6 \mathrm{~dB}$ and that the input impedance is mostly real for element lengths of several wavelengths [16]. 


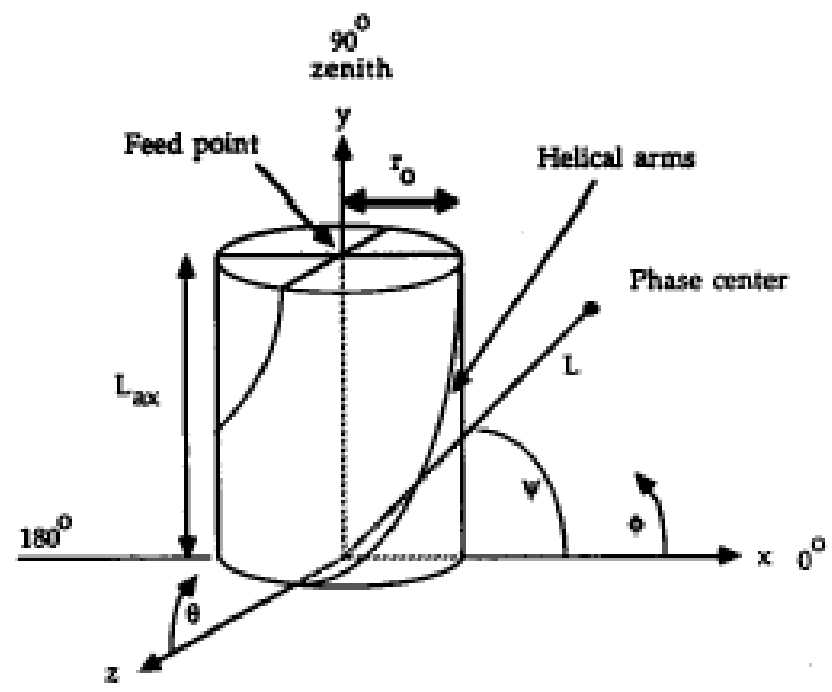

Figure 2.10: A quarter-turn resonant quadrifilar helical (volute) antenna.

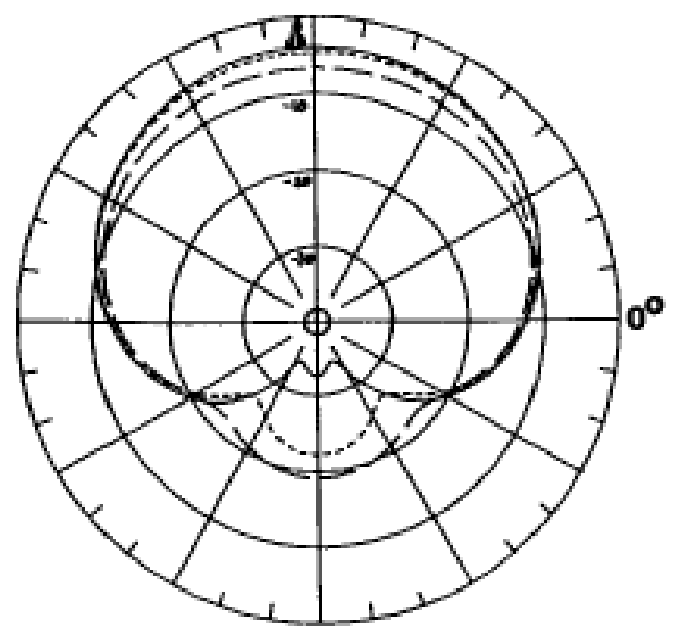

Figure 2.11: Computed half-turn volute antenna patterns on a logarithmic plot for various axial lengths. 


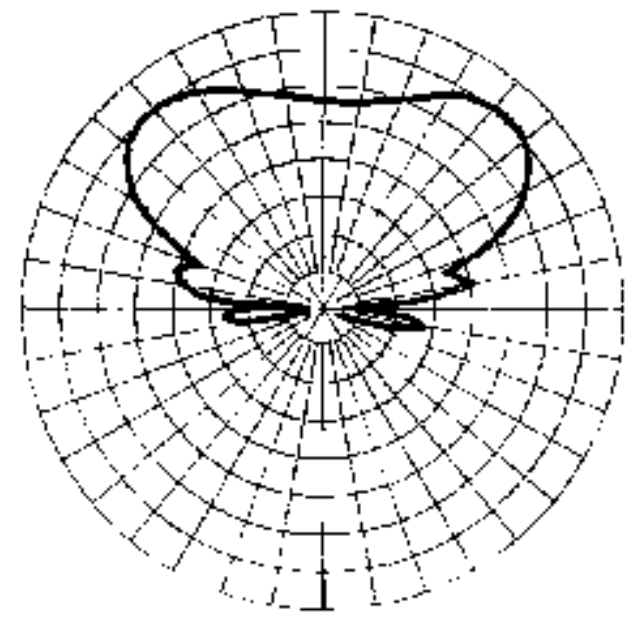

Figure 2.12: A log plot ( $5 \mathrm{~dB}$ per division) of the measured pattern of a quadrifilar helical antenna with 5 turns per element.

\subsubsection{The Bifilar Helical Antenna}

Similar to the quadrifilar helix is the bifilar helix, shown in Figure 2.13, which produces some similar properties using two elements instead of four [17]. The elements of the bifilar helix are fed in antiphase and produce a bifurcated beam, an example of which is depicted in Figure 2.14. 


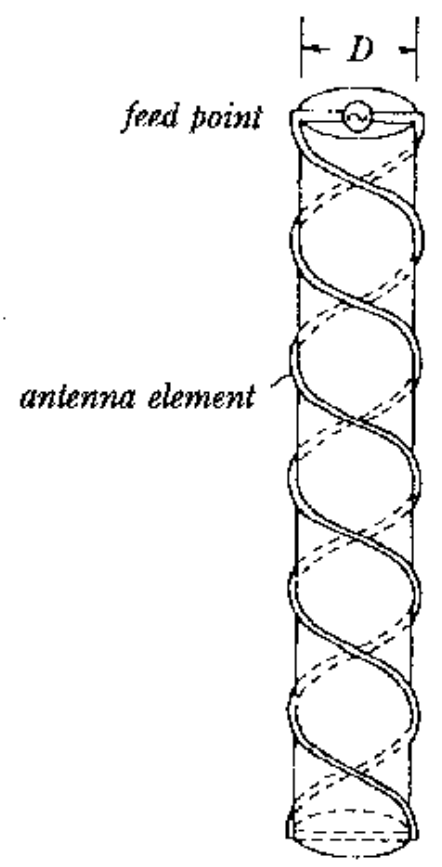

Figure 2.13: A bifilar helical antenna with 3 turns per element.

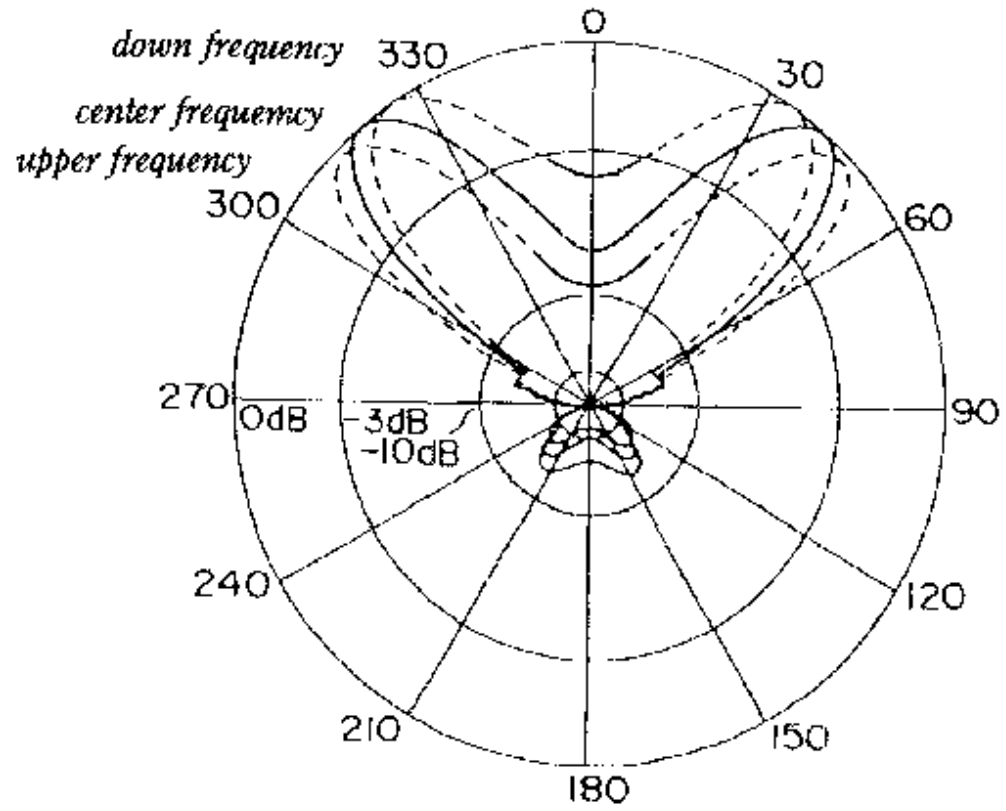

Figure 2.14: A log plot of the computed pattern for a bifilar helical antenna with 3 turns per element and an axial length of about $3 \lambda$ over a range of frequencies. 


\section{Chapter 3. The Spherical Helix}

The spherical helical antenna was first investigated by Cardoso $[9,10]$ and is shown in Figure 3.1. The spherical helix is a variation of the conventional helix that holds the spacing between turns (S) constant and varies the diameter (D) and pitch angle $(\alpha)$. Figure 2.9 shows this scheme in comparison with other methods of variation of the helix. The constant $\mathrm{S}$ condition implies that the spherical helix may be completely determined by the number of turns $(\mathrm{N})$ and the radius of the sphere (r), be it an actual or fictitious sphere, since each turn must traverse the same distance along the axis.

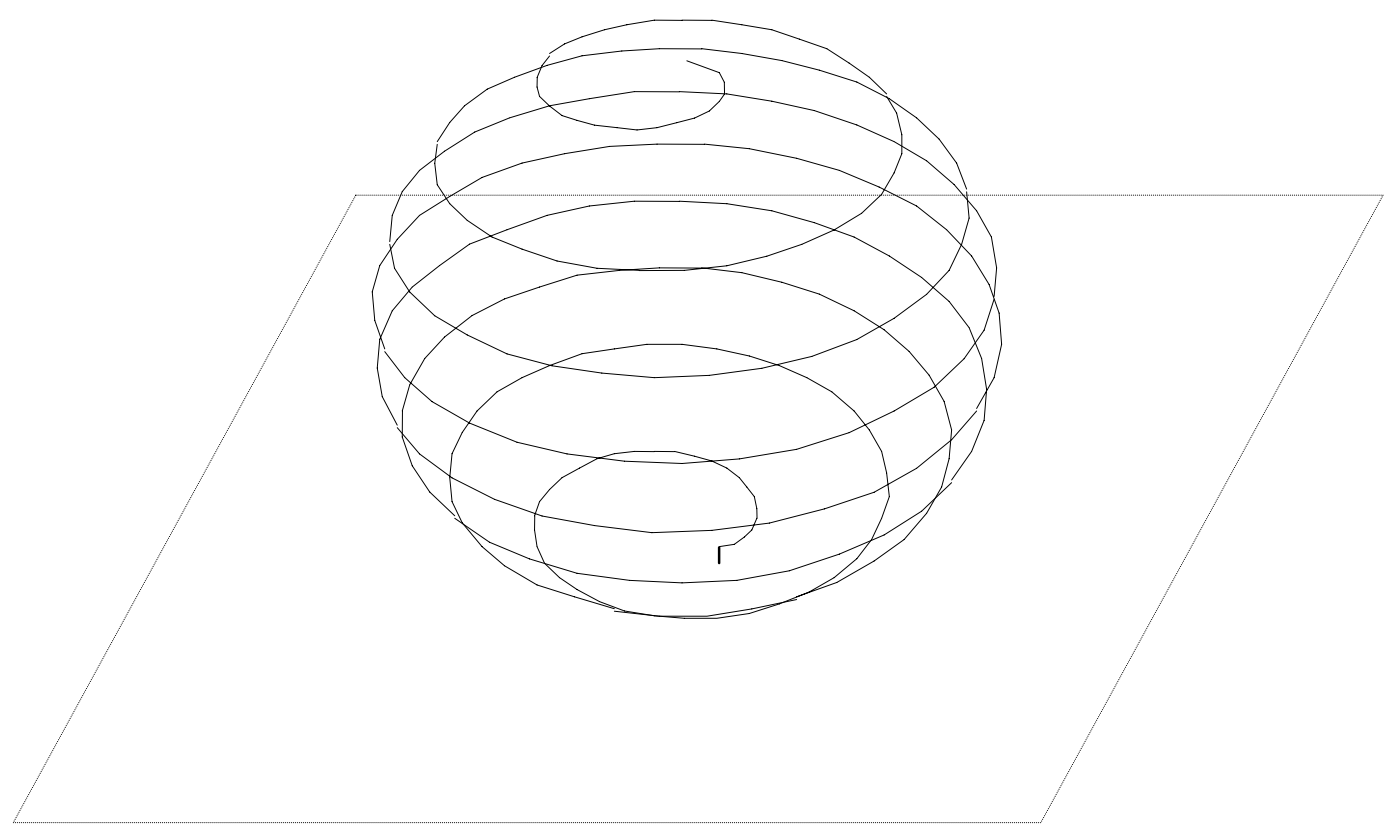

Figure 3.1: An 8-turn spherical helix over a square ground plane. A short feeding wire is present at the base. 
The geometry of the spherical helix is well known and may be summarized in equations (3-1) through (3-3) using spherical coordinates $(r, \theta, \varphi)$. It is, first of all, noteworthy that each turn sweeps out $2 \pi$ radians in the $\varphi$ coordinate, yielding a total of $2 \pi \mathrm{N}$ radians for the entire spherical helix of $\mathrm{N}$ turns. Then, as $\varphi$ varies from 0 to $2 \pi \mathrm{N}$ :

$$
\begin{aligned}
& \mathrm{r}=\mathrm{a} \\
& \theta=\cos ^{-1}\left(\frac{\phi}{N \pi}-1\right) \\
& 0<=\varphi<=2 \pi \mathrm{N}
\end{aligned}
$$

Since most numerical computation software (including NEC-4) requires a Cartesian coordinate $(\mathrm{x}, \mathrm{y}, \mathrm{z})$ representation of the antenna, it is useful to note the conversions from spherical to Cartesian coordinates in equations (3-4) through (3-6).

$$
\begin{aligned}
& x=r \sin \theta \cos \varphi \\
& y=r \sin \theta \sin \varphi \\
& z=r \cos \theta
\end{aligned}
$$

One advantage of the spherical helix over conventional cylindrical helices is the small electrical size. The axial length of the spherical helix is the same as the diameter, and thus the antenna is electrically very compact. For a spherical helix with a circumference of $1 \lambda$, the diameter is $0.3 \lambda$. The diameter, which is the primary physical dimension of the antenna, is then rather small electrically in the axial mode regime. 


\subsection{Radiation Characteristics of the Spherical Helix}

It has been found that the performance of the spherical helix is largely independent of the number of turns, excepting a very small number of turns where the helix loses its spherical shape and a very large number of turns where the helix becomes more like a spherical cavity [7]. It then becomes apparent that the radiation and impedance characteristics of the antenna are due largely to the spherical envelope of the helix.

The spherical helix was found to produce generally a large beamwidth (about $60^{\circ}$ for $3 \mathrm{~dB}, 110^{\circ}$ for $\left.10 \mathrm{~dB}\right)$ and moderately high axial gain $(9 \mathrm{dBi})$. The antenna radiation is elliptically polarized in general with circular or near-circular polarization under specific conditions over a large beamwidth but small bandwidth [10].

\subsubsection{The Axial and Axial-null Modes}

The spherical helix supports at least two modes of radiation in the overall axial regime: the so-called axial and axial-null modes. The axial mode is analogous to the axial mode of the standard helix, with a single main lobe along the axis of the helix. This regime operates for a circumference range of approximately $0.75 \lambda$ to $2.0 \lambda$. The axialnull mode operates for a normalized circumference range of about $2 \lambda$ to $2.8 \lambda$ [9]. The null that forms on the axis of the spherical helix in the axial-null mode can be accounted for by considering the currents on the wire. It turns out that the currents on opposite sides of the spherical helix are approximately $180^{\circ}$ out of phase, resulting in destructive interference along the axis. Figure 3.2 shows an example of a pattern for each radiation mode. 
In the axial-mode, the input impedance is complex and oscillatory. In the axialnull regime, the input impedance levels out at approximately 70 - j 50 Ohms. This corresponds to a VSWR of 2.4 for a $50 \Omega$ connecting cable.
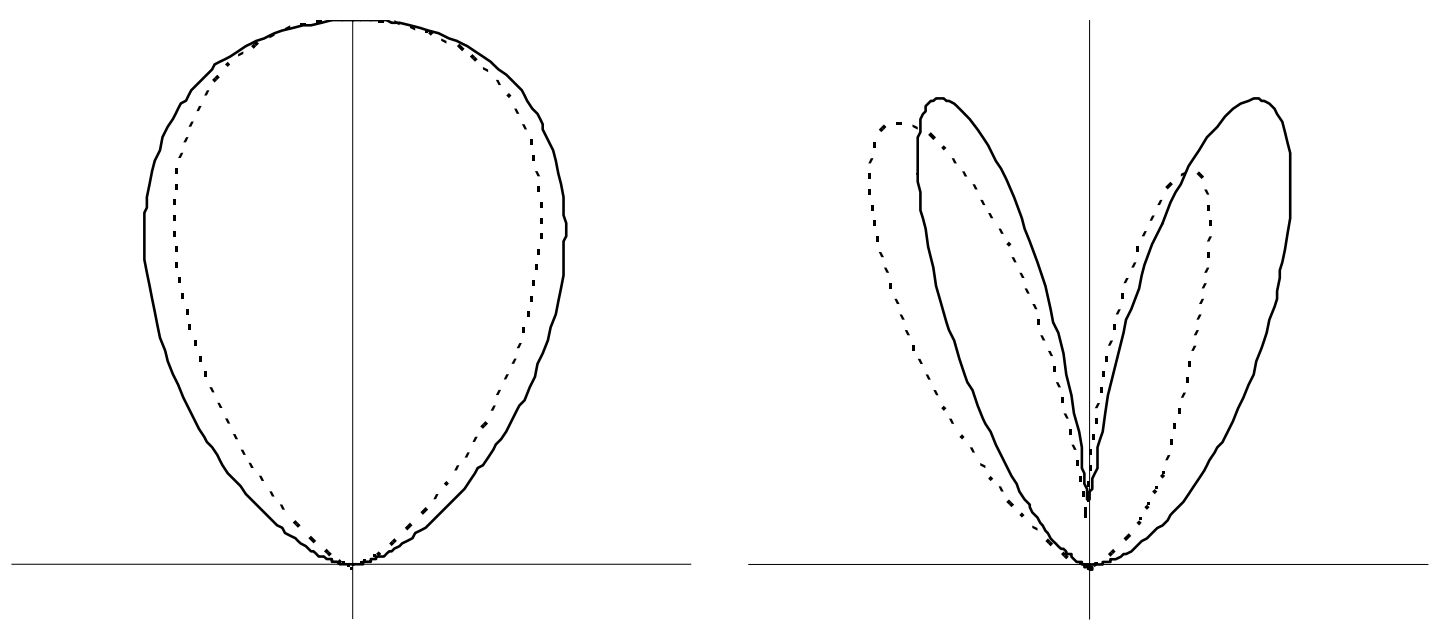

Figure 3.2: Radiation patterns simulated using NEC-4 for a 6-turn spherical helix with ground plane. The solid line is the $\varphi$ component, dashed is the $\theta$ component. The plots are on a linear scale. On the left, the normalized circumference is $1.1 \lambda$ (axial mode), on the right, $2.2 \lambda$ (axial-null mode).

Overall, the spherical helix provides an appealing alternative to the conventional (cylindrical) helix, due to its compact electrical size combined with many of the radiation characteristics of the conventional helix. Some drawbacks to this configuration include its mechanical instability, since, if it lacks additional supports, the antenna may be easily bent or broken. Additionally, it lacks the nearly purely real input impedance of the conventional helix. This, combined with the oscillatory nature of the impedance with frequency, limits the effective bandwidth due to variation in the amount of radiation over a given frequency range (especially in the axial mode). 


\subsection{The Truncated Spherical Helix}

The spherical helix may be 'truncated' by simply varying $\varphi$ over some lesser portion of the range from 0 to $2 \pi \mathrm{N}$, as shown in Figure 3.3. The notion of the "truncated spherical helix" is integral to the investigation of the hemispherical helix in later chapters. In accordance with the nomenclature of Cardoso, a truncated spherical helix of radius $a$ may be represented by the number of turns in the actual antenna, $n$, and the number of turns in the corresponding full helix, N. The standard spherical helix is a limiting case of the truncated helix, with $\mathrm{n}=\mathrm{N}$, while all other cases have $\mathrm{n}<\mathrm{N}$. Additionally, the spherical helix may be extended to include more than one full sphere, in which case it might be appropriate to represent it with $\mathrm{n}>\mathrm{N}$.

The truncated spherical helix was investigated by Weeratumanoon $[18,19]$. The truncation in this case, however, involved removing a portion of the top of a standard spherical helix. This configuration was found to yield, with an optimum number of turns ( $\mathrm{N}$ and $\mathrm{n}$ ), improved bandwidth and axial ratio over that of the standard spherical helix [18]. Other radiation characteristics remained the same. This provided improved performance and a more compact size. Once again, however, the truncated spherical helix lacks mechanical stability, making it less desirable. 


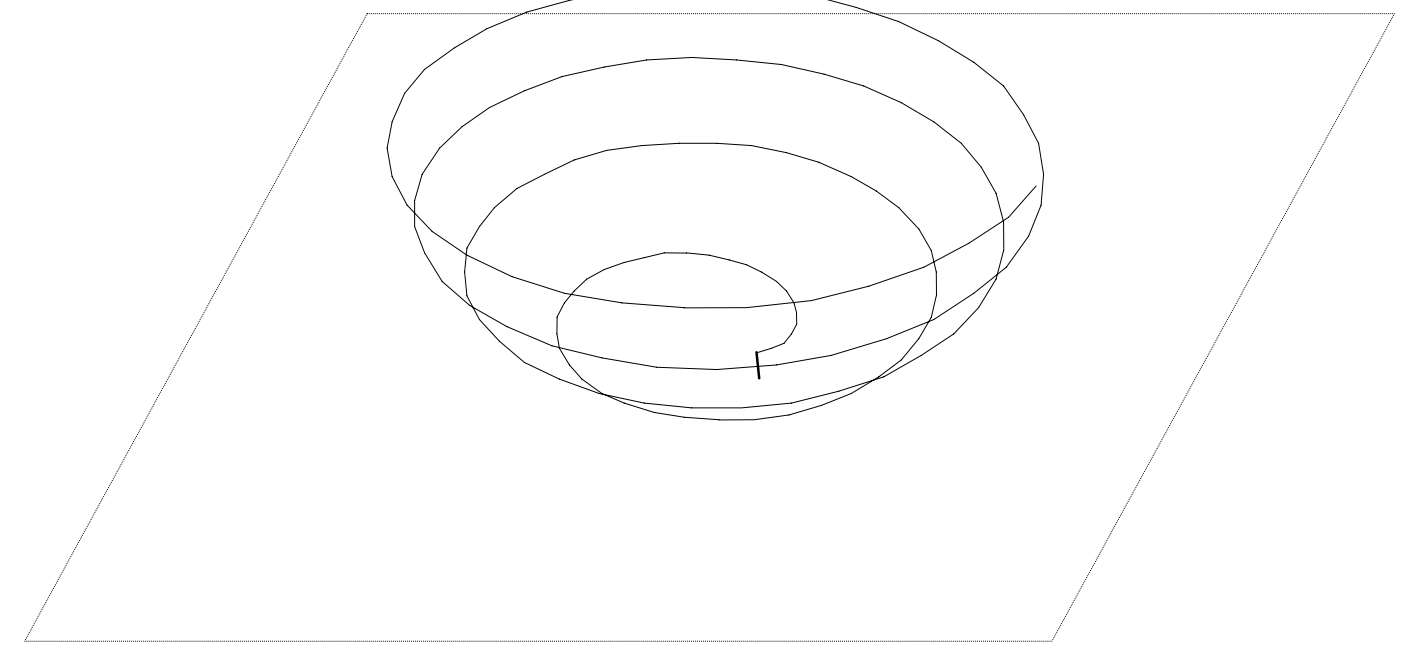

Figure 3.3: A truncated spherical helix, with $\mathrm{N}=8$ and $\mathrm{n}=5$, with feeding wire over square ground plane. 


\section{Chapter 4. The Multifilar Hemispherical Helix}

The hemispherical helix is a variation of the standard spherical helix that consists simply of the top half of the spherical helix. This variation of the spherical helix has been investigated $[20,21]$ and shown to have many of the same properties as its predecessor while providing an improvement in both mechanical stability and electrical size. It is shown in this investigation that further modifications yield improvements and additional features in the radiation characteristics of the antenna.

The geometry of the hemispherical helix is similar to the spherical helix and is expressed in equations (4-1) through (4-3), where $\mathrm{n}$ is the number of turns.

$$
\begin{aligned}
& \mathrm{r}=\mathrm{a} \\
& \theta=\cos ^{-1}\left(\frac{\phi}{N \pi}-1\right) \\
& 2 \pi \mathrm{n}<=\varphi<=2 \pi \mathrm{N}
\end{aligned}
$$

If $\mathrm{N}$ is the number of turns in a corresponding standard spherical helix, then $\mathrm{n}$ is the same as $N / 2$. The hemisphere may be placed directly against the ground plane (if used) and results in an extremely stable, compact configuration.

\subsection{Feeding Methods}

The hemispherical helix may be fed in a number of ways. These include bottomfed (Figure 4.2), top-fed (Figure 4.3) and side-fed (Figure 4.1) configurations. Top-fed 
and bottom-fed hemispheres involve a wire from the feed point (at the origin) directly either upward to the apex (top) of the hemisphere or across to the termination of the helix near the ground plane (base). A side-fed hemisphere involves translation of the helix such that the termination of the wire is at the feed point.

\subsubsection{Bottom-feeding}

A bottom fed hemisphere maintains symmetry around the origin (and feed point) and, thus, perhaps most closely resembles the standard spherical helical geometry. Since a wire segment is required to connect the feed to the base of the helix, there is a significant portion of the antenna that is very close to the ground plane. This produces an image that tends to suppress radiation and lower the gain of the antenna but eliminates the contribution of the connecting segment. Figure 4.1 shows a bottom-fed hemispherical helix. This feeding method has also been called 'central-fed' and has been investigated by Chan, Hui and Yung [20].

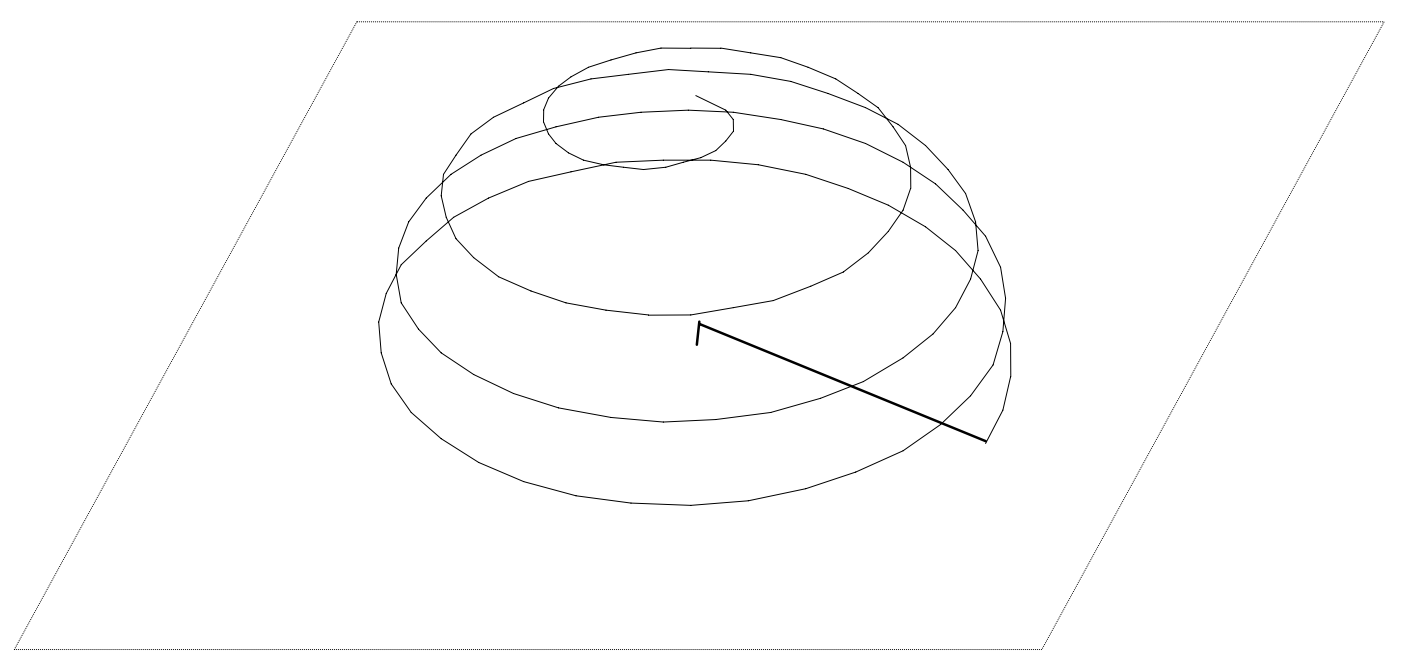

Figure 4.1: A bottom-fed hemispherical helix with feeding wire parallel to ground plane. 


\subsubsection{Top-feeding}

A top-fed hemisphere has the added benefit of not having any wires close to and parallel with the ground plane. The connecting wire segment maintains an approximately cylindrical symmetry about the helical axis. The difficulty with this configuration is that the connecting wire disturbs the radiation characteristics of the pure hemispherical helix. It is hoped that the disturbance is minimal or beneficial to the performance of the antenna. Additionally, the current distribution along the helix is different since the helix is fed from the top as opposed to the bottom. Figure 4.2 shows a top-fed hemispherical helix.

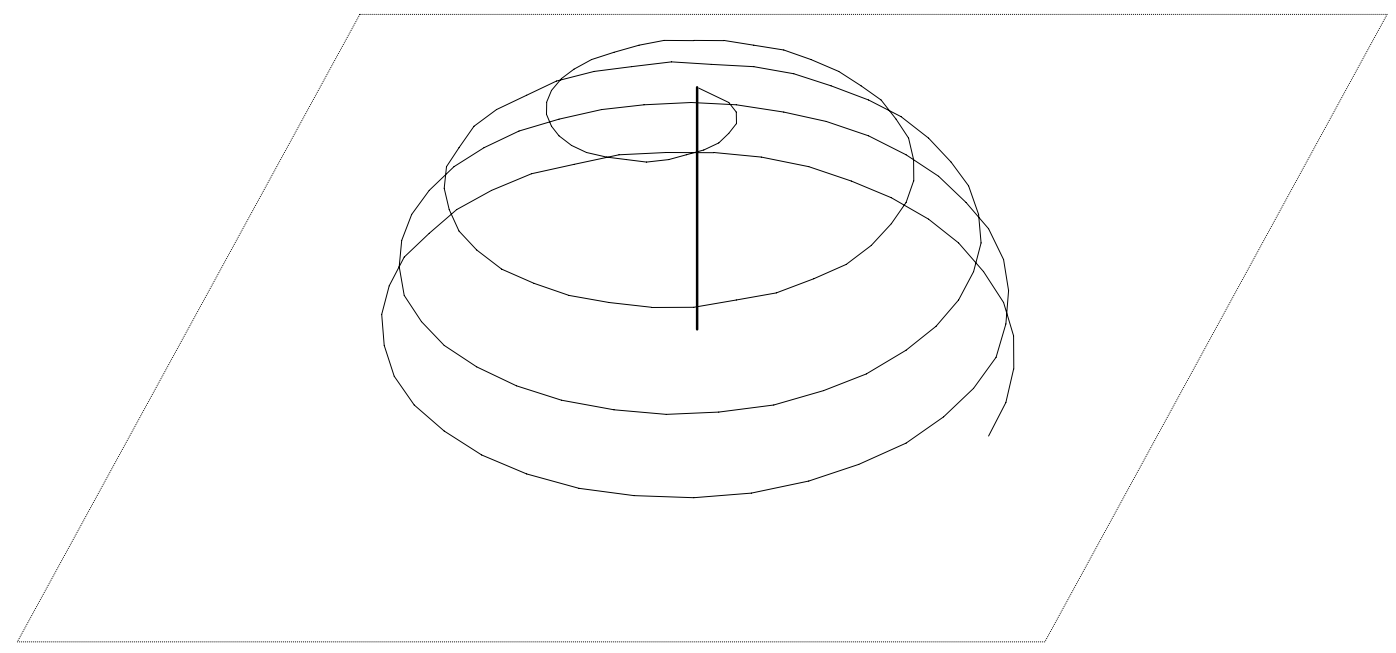

Figure 4.2: A top-fed hemispherical helix with feeding wire perpendicular to ground plane.

\subsubsection{Side-feeding}

The side-fed hemisphere eliminates the difficulties of the top-fed and bottom-fed hemispherical helices. The translation of the hemisphere eliminates the necessity of a 
significant connecting wire between the feed point and the helix (some very small connecting segment may be required). Although the feed point is no longer the origin of the helix, resulting in the destruction of symmetry, it is likely that this has little effect on the performance of the antenna, especially if the ground plane is electrically large. A side-fed hemispherical helix is shown in Figure 4.3. The hemispherical helix with this feeding method has been investigated (a 'coaxial-feed' antenna) [21].

Figure 4.3: A side-fed 4-turn hemispherical helix with square ground plane and short feeding wire.

\subsection{Multifilar Hemispherical Helices}

A spherical or hemispherical helix may be modified by wrapping multiple wires around the same hemisphere. This yields a multiple-arm (or multifilar) hemispherical helix. It is anticipated that this variation will improve the radiation characteristics and significantly lower undesirable qualities. It is noteworthy that a multifilar hemispherical 
helix has been investigated by Best [22]. In this case, however, the antenna was electrically very small.

\subsection{Simulation of Multifilar Hemispherical Helices Using NEC-4}

Multifilar hemispherical helices were investigated using NEC-4 for two, three and four arms arranged symmetrically around the helical axis. Each of these three configurations was examined for both side-feeding and top-feeding. Bottom-feeding was not pursued due to the previously mentioned disadvantages. Since the helical circumference may be normalized to wavelengths, the number of turns in the antenna is the other parameter of interest. For the two-arm (bifilar) hemisphere, antennas with 1,2 or 3 turns per arm were simulated. Likewise, for the three-arm (trifilar) hemisphere, 1, 1.5 and 2 turns per arm were examined, and for the four-arm (quadrifilar) hemisphere, $0.5,1,1.5$ and 2 turns per arm were examined. Arms with a larger number of turns than studied here were ignored due to the fact that spherical helices with a very large number of turns begin to take on the behavior of a spherical cavity [7].

While some asymmetric configurations of the above were looked into, of primary interest are the symmetric multifilar hemispherical helices. In each case, at the apex of the hemisphere, all arms emerge at equal angles from each neighboring arm, resulting in a rotational symmetry of $180^{\circ}$ for bifilar, $120^{\circ}$ for trifilar and $90^{\circ}$ for quadrifilar helices.

The diameter of the conductor, while a relevant parameter for the performance of these antennas, was not examined in depth. A brief investigation, however, revealed that the VSWR and axial ratio may both be improved by altering the diameter of the conductor. Once these antennas were simulated and the results examined, there 
emerged two particular designs with significant properties: the top-fed bifilar and sidefed quadrifilar hemispherical helices. Chapter 5 details the construction and measurement of prototypes of these particular antennas.

\subsection{The Top-fed Bifilar Hemispherical Helix}

The bifilar top-fed hemispherical helix involves a standard hemispherical helix with an identical helix rotated $180^{\circ}$ about the axis, as shown in Figure 4.4. This variation of the hemispherical helix operates entirely in the axial-null mode due to the symmetry about the helical axis. Any current increment on one arm of the helix is matched by an equivalent current increment on the opposite arm. This is depicted in Figure 4.5. Since these increments are $180^{\circ}$ out of phase, the radiated fields interfere destructively along the axial direction, yielding a null in the pattern at bore sight. Unlike the spherical helix, which operates in axial-null mode only for certain frequencies, the two-arm symmetric hemisphere operates in axial-null mode for all frequencies due to its symmetry. This yields a very large bandwidth for axial-null mode operation. If the bifurcated pattern of the axial-null mode is desirable, then there is a large frequency range available with this antenna for finding other advantageous radiation characteristics such as circular polarization or low VSWR at the feed point. 


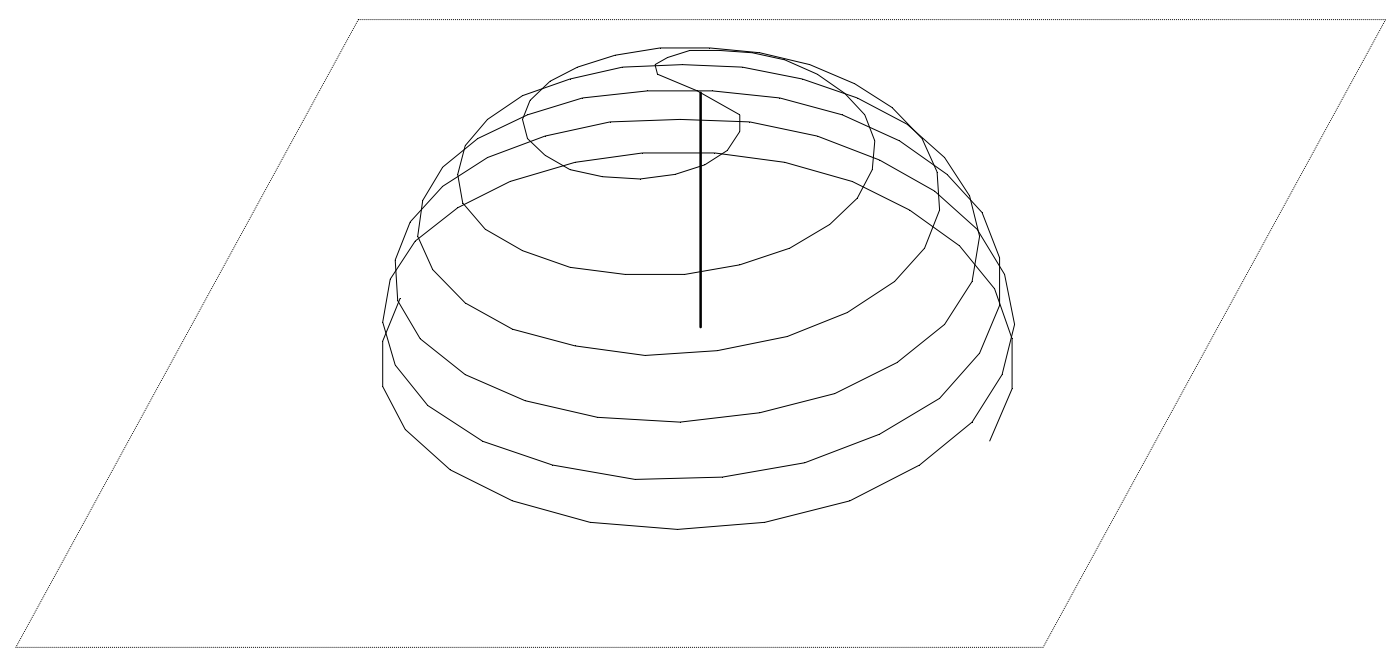

Figure 4.4: A top-fed, bifilar (two-arm) hemispherical helical antenna with square ground plane.

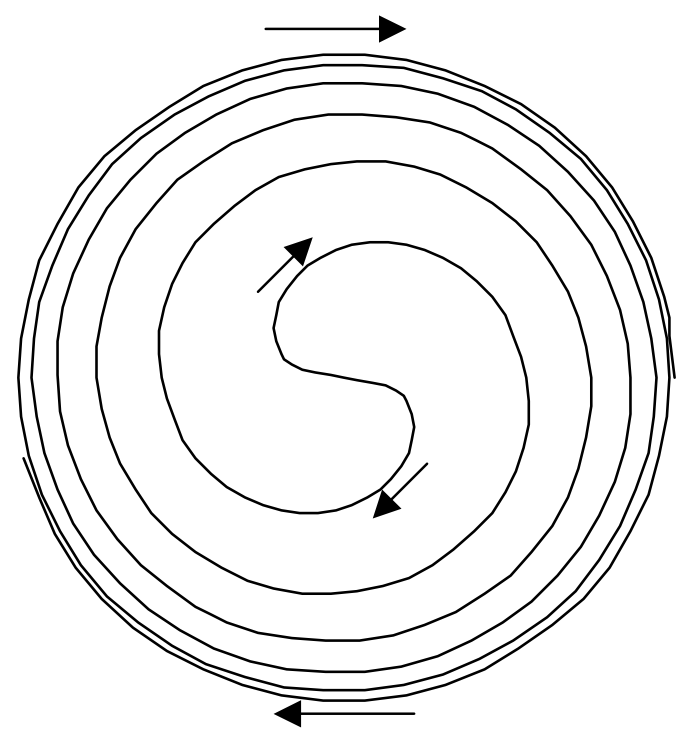

Figure 4.5: A top view of the top-fed bifilar hemispherical helix with current directions shown at several points. 
The bifilar hemispherical helices were simulated for normalized circumferences of $0.5 \lambda$ to $3.5 \lambda$ in increments of $0.1 \lambda$. The bifurcated pattern of the axial-null mode was seen at all frequencies. A typical pattern is shown in Figure 4.6; Appendix A contains the patterns obtained in the simulations. Some 'wagging' of the main lobes occurred, but was fairly limited. The diameter of the conductor used in the simulation was $0.321 \mathrm{~mm}$, corresponding to 28 gauge wire. This value was chosen for convenience and ease of construction of the antenna for measurement purposes, as thicker wire quickly becomes very unwieldy.

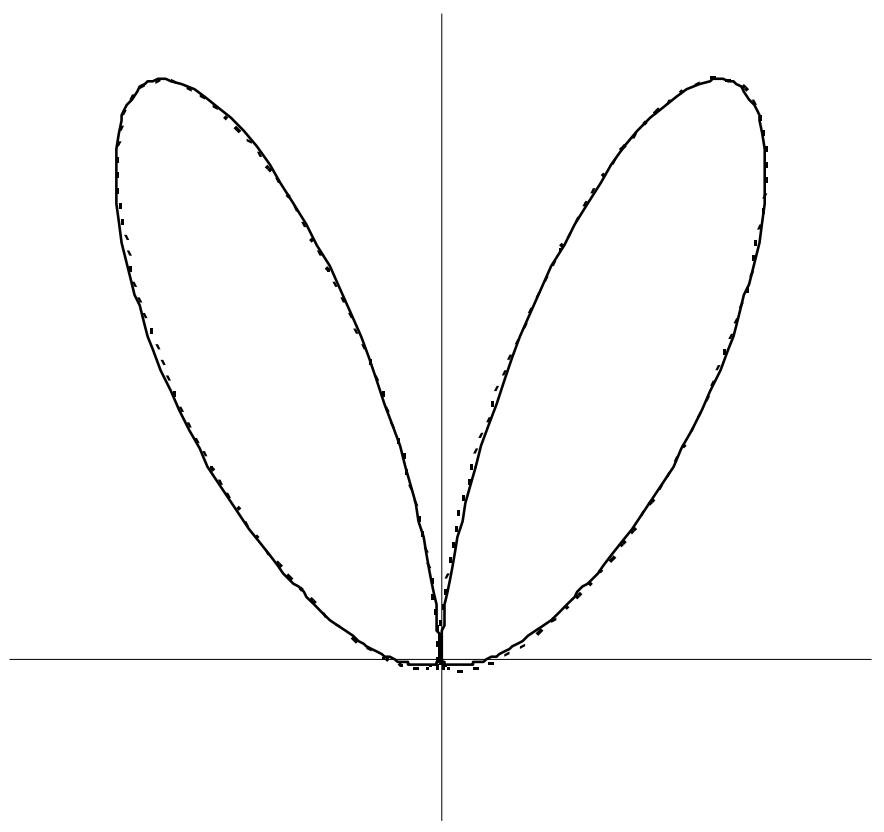

Figure 4.6: The simulated radiation pattern in the E-plane for the bifilar hemispherical helix at a normalized circumference of $2.86 \lambda$. The $\theta$ (dashed) and $\varphi$ (solid) components of the field were normalized to the same maximum and nearly coincide.

Since a very high VSWR at the feed point of the antenna is detrimental to the amount of radiation emitted, it is appropriate to concentrate on frequency ranges with a low VSWR. For the antenna under investigation, the range of interest corresponds to a 
normalized circumference range of about $2.5 \lambda$ to $3.1 \lambda$, as can be seen in Figure 4.7. It was found that, in this range, the polarization is elliptical in general and circular in particular over limited beamwidths at some frequencies. Also of interest were those frequencies which produced circular polarization, which were limited to the range of approximately $2.7 \lambda$ to $3.1 \lambda$. A plot of the axial ratio and phase difference between the $\theta$ and $\varphi$ components of the electric field for the antenna in the H-plane is shown at one frequency in Figure 4.8.

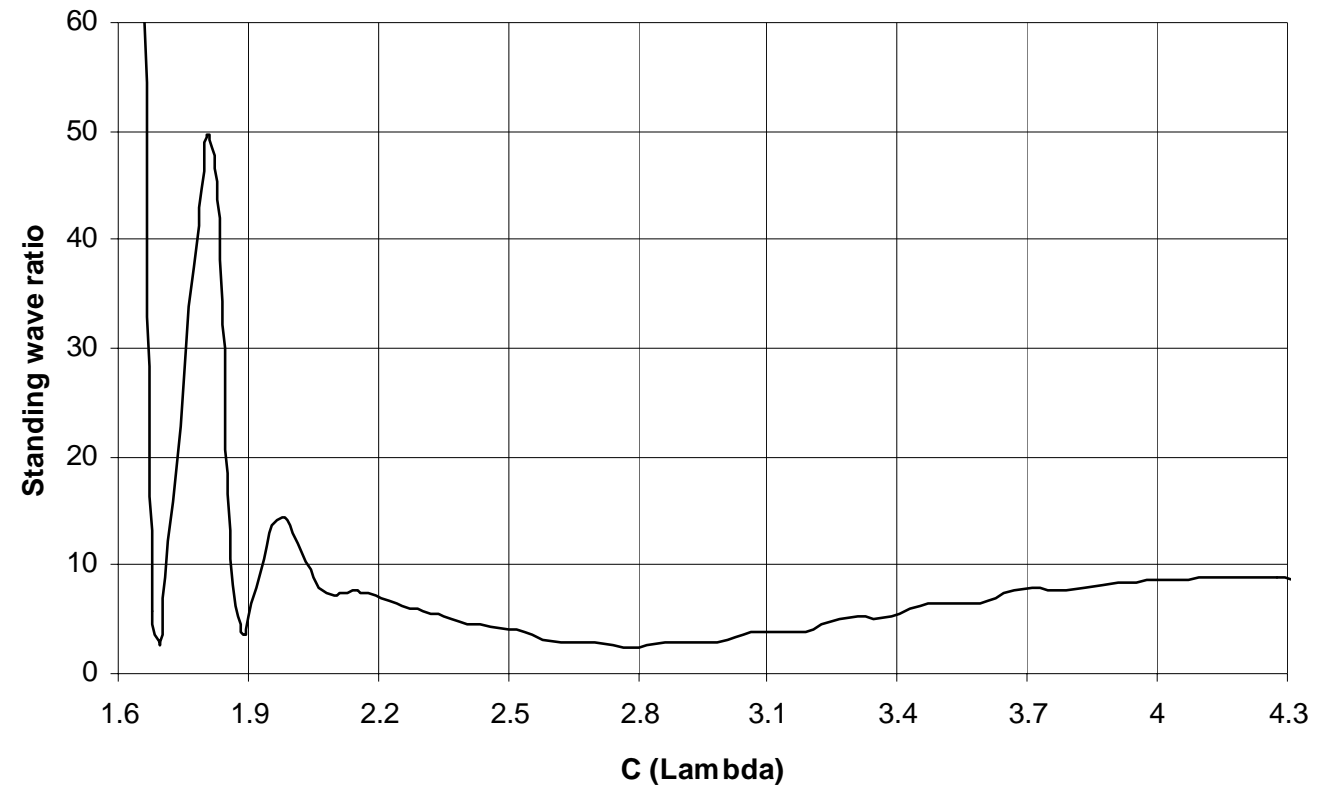

Figure 4.7: The simulated VSWR for the bifilar hemispherical helix. 

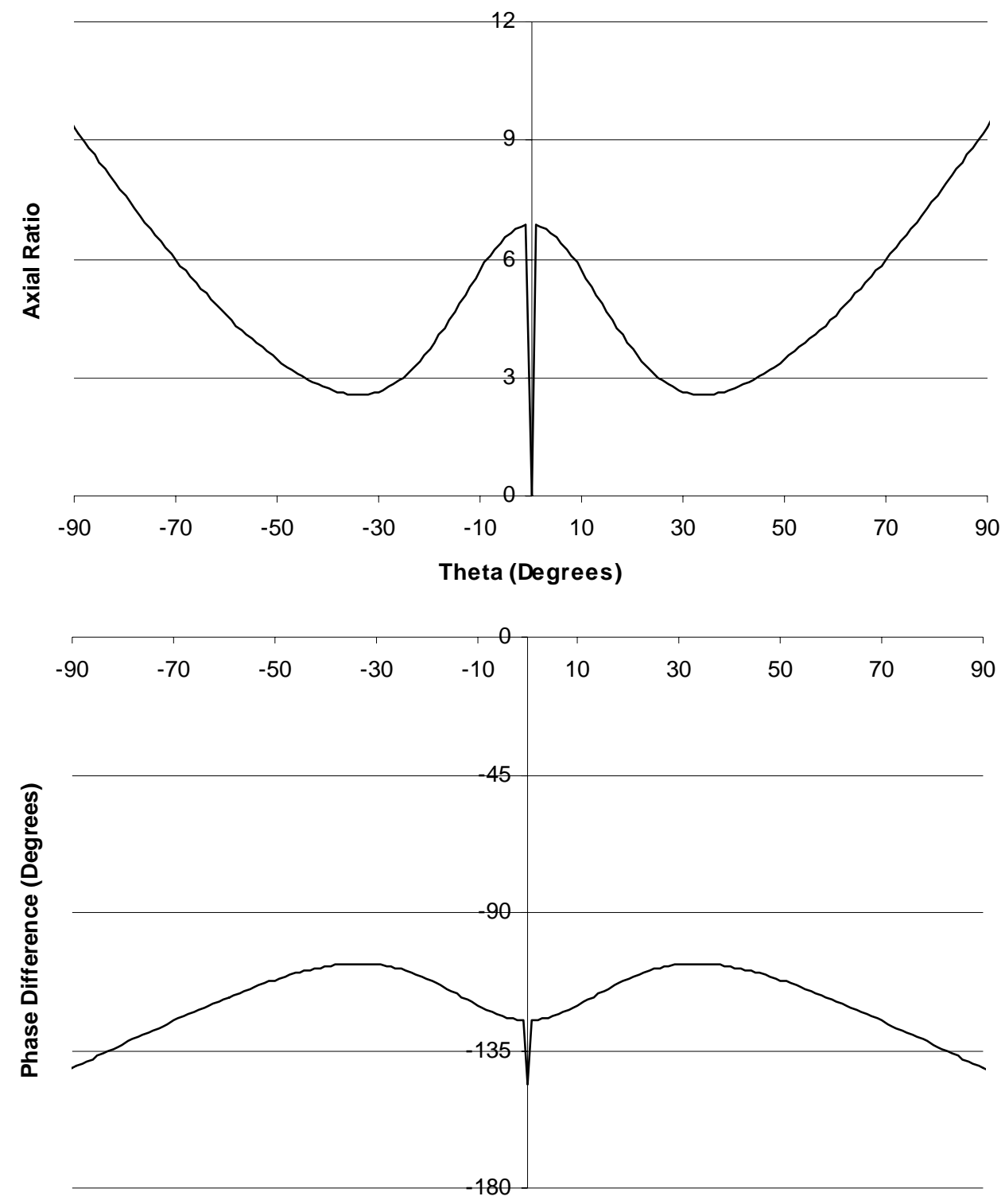

Figure 4.8: The axial ratio (top) and phase difference (bottom) in the H-plane for the bifilar hemispherical helix at a normalized circumference of $2.90 \lambda$.

\subsection{The Side-fed Quadrifilar Hemispherical Helix}

The side-fed quadrifilar hemispherical helix is a variation that involves two bifilar hemispheres at $90^{\circ}$ angles at the apex of the hemisphere. Figure 4.9 shows an example of this antenna. Using a side-feed on one arm, it is expected that the operation of this 
antenna will differ vastly from the top-fed bifilar hemisphere, since there will not be equal currents on each arm. Since the hemispherical helix is a traveling-wave antenna, it is likely that the current at the apex of the hemisphere will be low with respect to the feed point. The remaining current will likely be distributed among the other three arms or in reflection. It would appear, based on a brief evaluation, that this antenna will act largely like the standard hemispherical helix. It is hoped that the additional arms will improve the symmetry of the radiation patterns and possibly improve the polarization.

The quadrifilar hemispherical helix was simulated using NEC-4 over a normalized circumference range of $0.5 \lambda$ to $2.3 \lambda$ in increments of $0.1 \lambda$. As in the simulations of the bifilar antenna, 28 gauge conductor was used.

Unlike with the bifilar hemispherical helix, the quadrifilar has no significant bandwidth with a reasonable VSWR, as shown in Figure 4.10. However, several resonances are present in which the VSWR falls below 2. Since method of moments codes are not always reliable for VSWR readings, it is possible that measurement may yield some more acceptable values.

It was found that this antenna produced largely linear polarization and a symmetrical axial beam over a reasonable bandwidth. An example pattern is shown in Figure 4.11 and the axial ratio and phase difference for that pattern in Figure 4.12.

It is noteworthy that the E- and H-planes have been defined in terms of the geometry of (4-1) through (4-3). The H-plane is the plane of $\varphi=2 \pi n$, while the E-plane is $\varphi=2 \pi \mathrm{n}+\pi / 2$. The measurements of the prototypes, discussed later, were made with this definition in mind. 


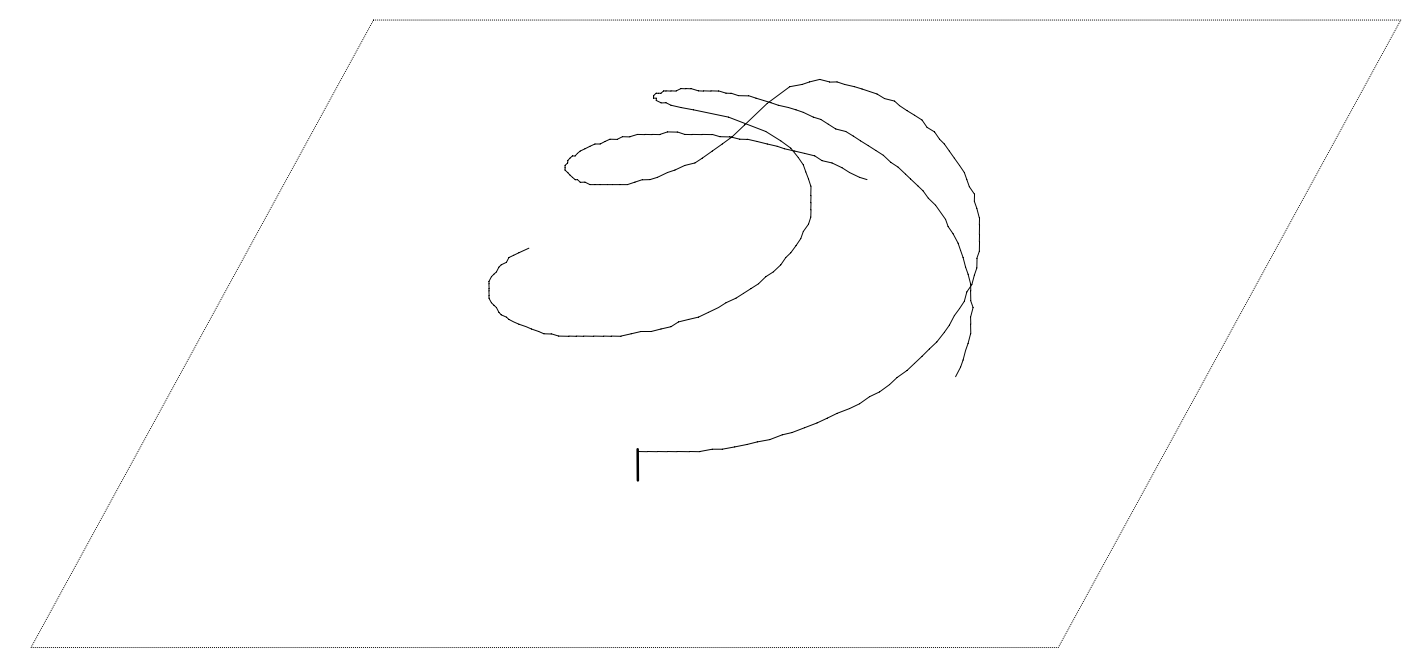

Figure 4.9: The side-fed quadrifilar hemispherical helix with one turn per arm.

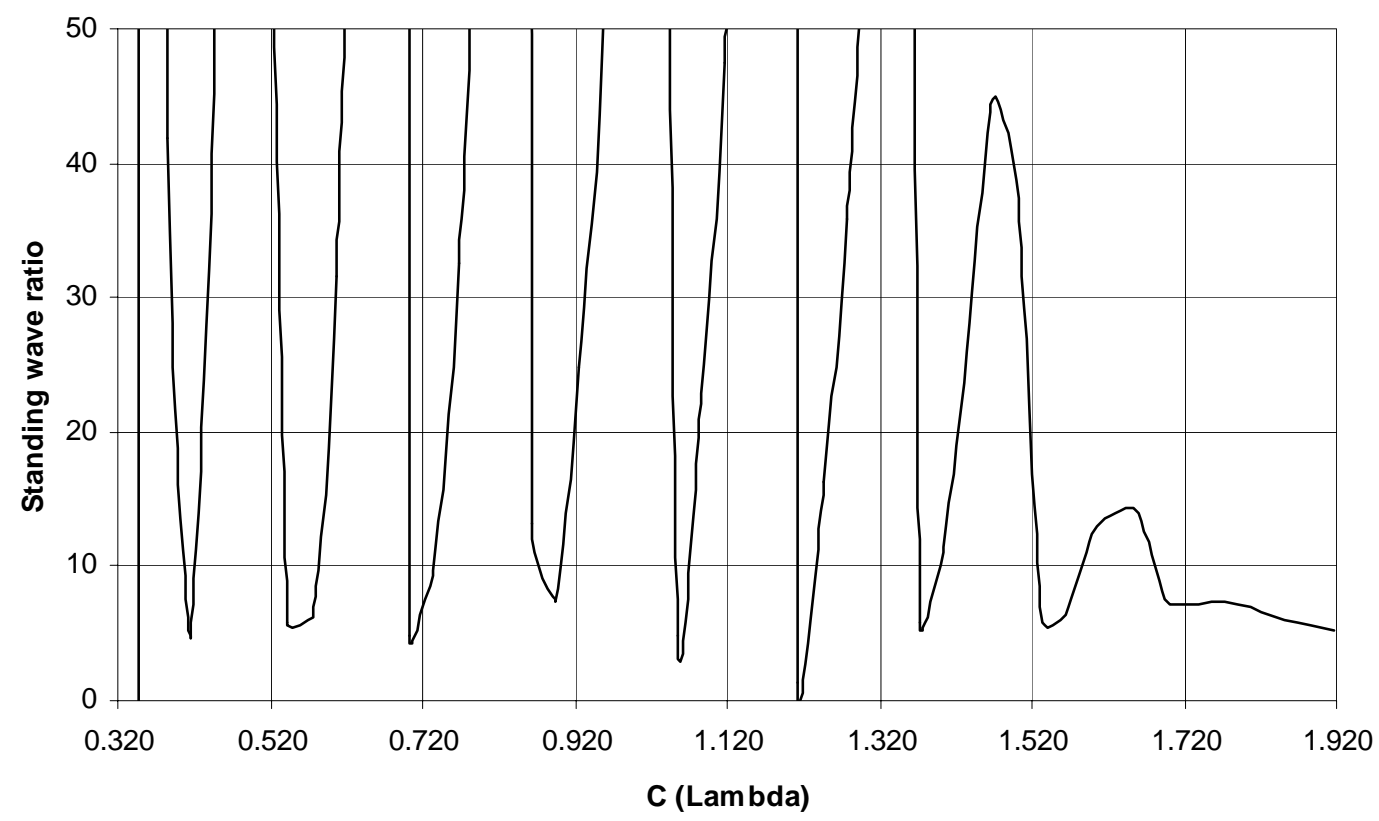

Figure 4.10: Simulated results for VSWR of the quadrifilar hemispherical helix. 


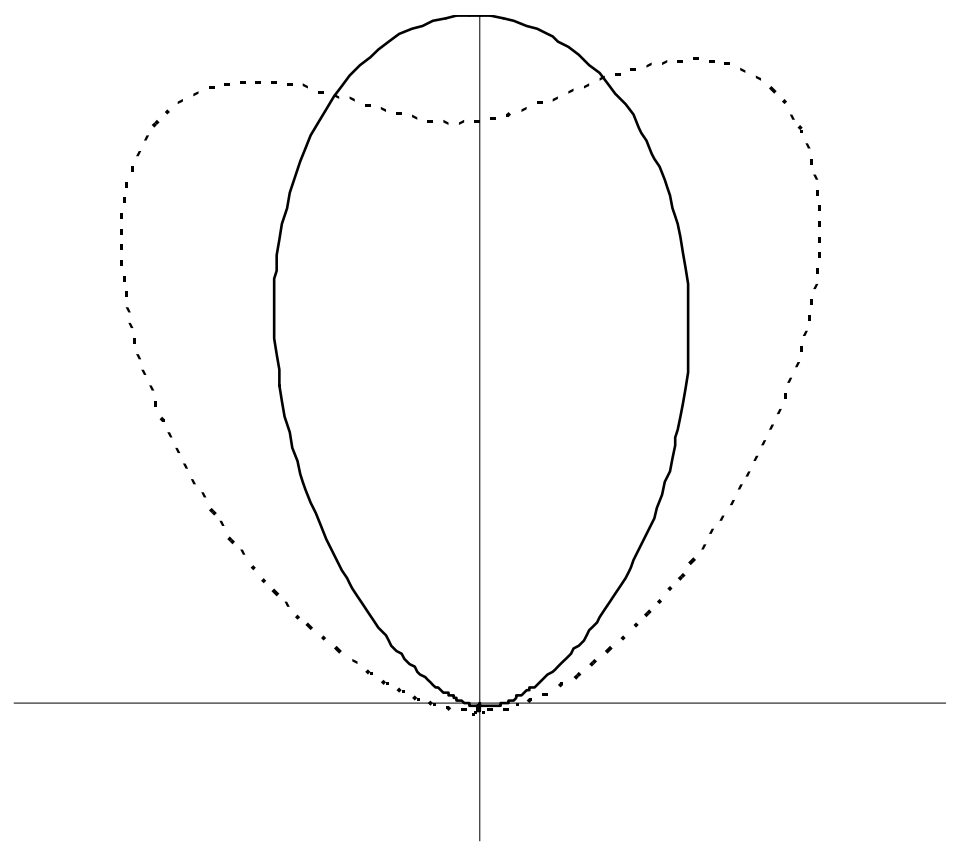

Figure 4.11: The simulated radiation pattern in the E-plane for the quadrifilar hemispherical helix at a normalized circumference of $0.80 \lambda$. Shown are the $\theta$ component (dashed) and $\varphi$ component (solid) of the field. 

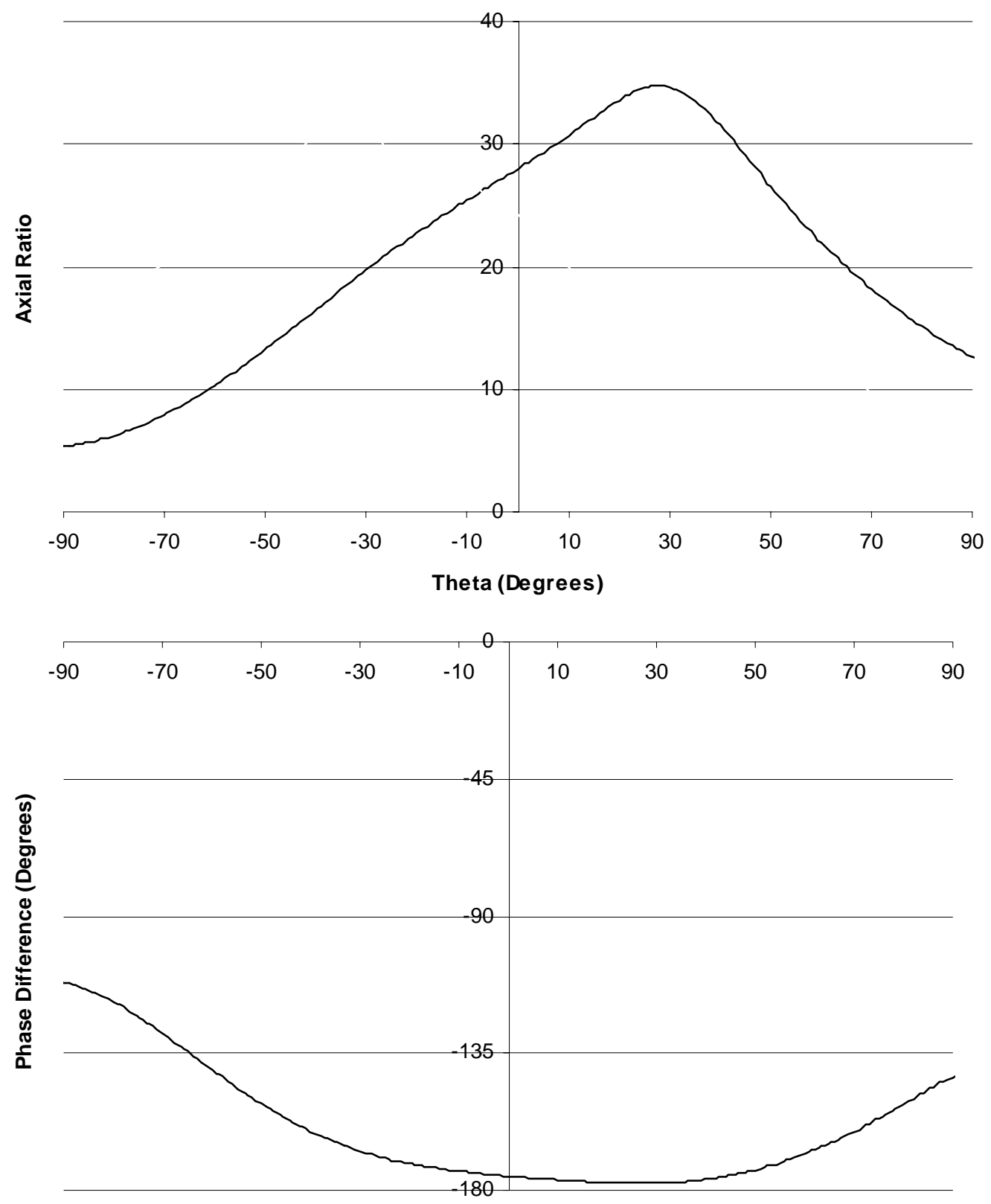

Figure 4.12: The axial ratio (top) and phase difference (bottom) in the E-plane for the quadrifilar hemispherical helix at a normalized circumference of $0.80 \lambda$. 


\section{Chapter 5. Prototype Construction and Measurement}

To confirm the simulation results and identify shortcomings in the software used, prototypes of the hemispherical helices investigated in the previous chapter are built and studied experimentally. The measurements are particularly useful in assessing the VSWR performance of the antennas, as the accuracy of NEC-4 results for the input impedance is in doubt.

Prototype models of the top-fed bifilar and bottom-fed quadrifilar hemispherical helices were constructed following the approach of Cardoso [7]. Styrofoam spheres of diameter $7.4 \mathrm{~cm}$ and $6.1 \mathrm{~cm}$, respectively, were used as the support for the wire antennas. An equator was marked and each sphere was cut in half to form a hemisphere. For the bifilar helix, a hole was drilled through the apex to the center, thus making a space for the feed wire.

\subsection{Construction Procedures}

For the bifilar helix, a single long piece of 28 gauge wire served as two arms of the helix and was soldered, at its midpoint, to a second piece of wire which served as the feed wire. For the quadrifilar helix, two long pieces were soldered at their midpoints, forming the four arms. One arm was left slightly longer than needed to allow a small feeding segment. This is illustrated in Figure 4.1. Superglue was used as the adhesive agent, and it is assumed for this investigation that it has a negligible effect on the performance of the antenna, since it constitutes only very thin layers near or around the 
wire. Additionally, Styrofoam serves as a useful material since it is largely composed of air and thus has a dielectric constant very close to that of the atmosphere $(\approx 1)$.

After securing the wires of the helices to the hemispheres, the feed wires were then attached to ground planes using coaxial connectors. A metal pie plate of radius 21.5 $\mathrm{cm}$ for the bifilar helix provided a convenient ground plane due to its availability and low cost. Figure 5.1 shows the prototype mounted in the anechoic chamber. Some thin pieces of cardboard were used to support the hemisphere, as it remained several millimeters above the ground plane. For the quadrifilar helix, a pizza plate of radius 40 cm was used. The helix was held in place by some clear tape, as shown in Figure 5.2.

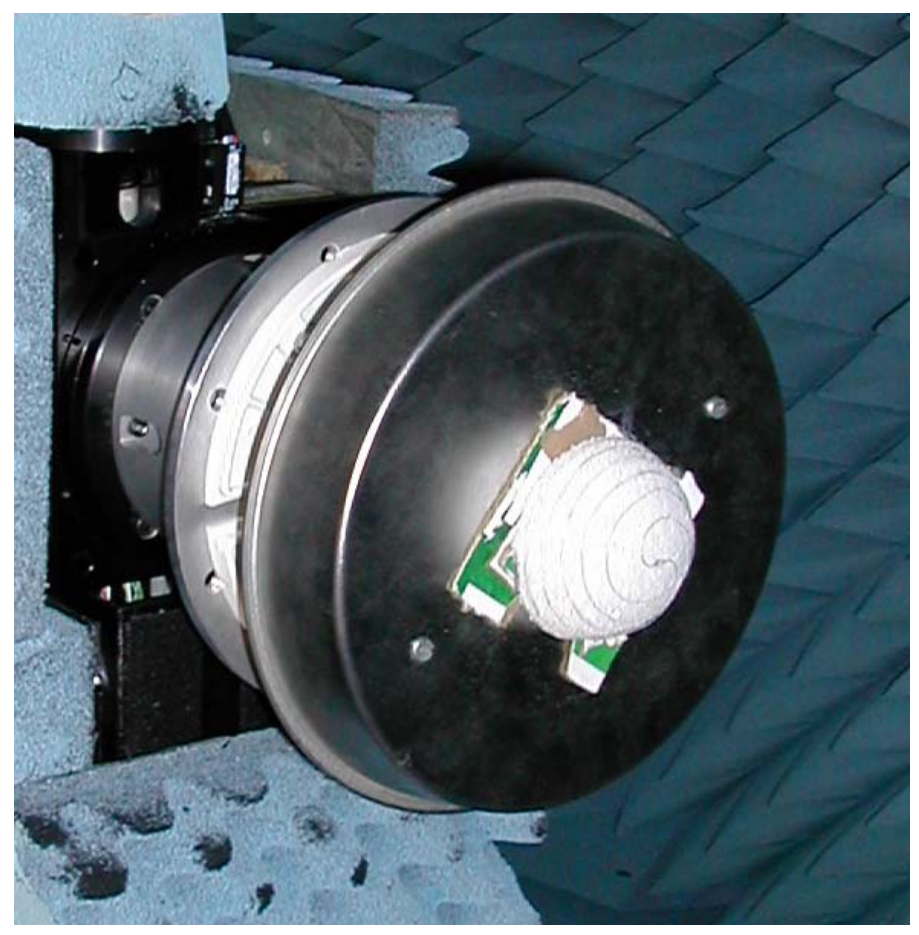

Figure 5.1: The prototype bifilar hemispherical helix with ground plane, mounted in the anechoic chamber for measurements. 


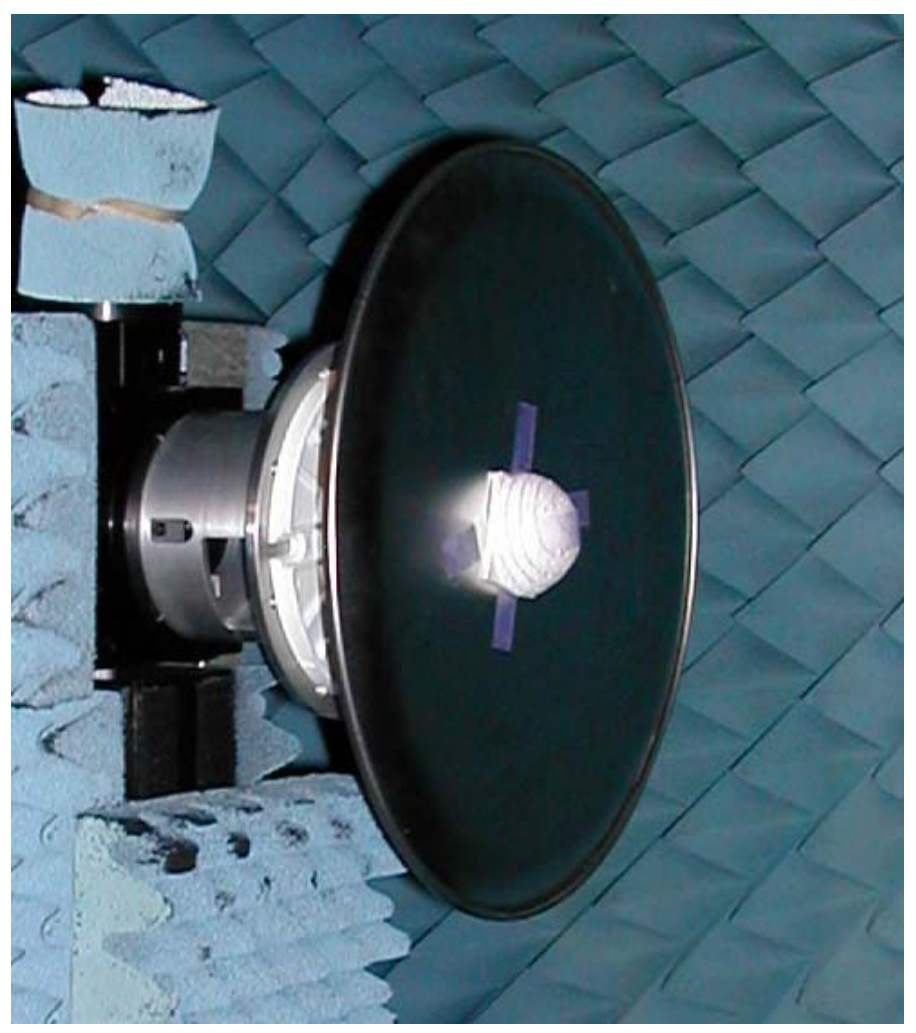

Figure 5.2: The quadrifilar hemispherical helix with ground plane mounted in the anechoic chamber.

\subsection{Pattern Measurement Setup}

The radiation characteristics of the antennas were measured at the Virginia Tech Antenna Group's indoor antenna range. A series of near field measurements were taken and then transformed by the controlling software package to yield the far fields at various frequencies. An open ended waveguide was used as the detector, as shown in Figure 5.3, and the entire apparatus was enclosed in an anechoic chamber to reduce multipath reflections. The data collected included both the magnitude and phase of the theta and phi components of the electric field in the far zone in both the E- and H-planes. The availability of phase information in the measurements allowed for determination of the 
polarization of the radiation [6], a critical factor in the investigation. The frequencies at which data were collected for the bifilar hemispherical helix were in the region of low VSWR, namely from 3.4 to $3.9 \mathrm{GHz}$ in $50 \mathrm{MHz}$ increments. Only several token measurements were taken of the quadrifilar helix, from 1.15 to $1.25 \mathrm{GHz}$ in $50 \mathrm{MHz}$ increments.

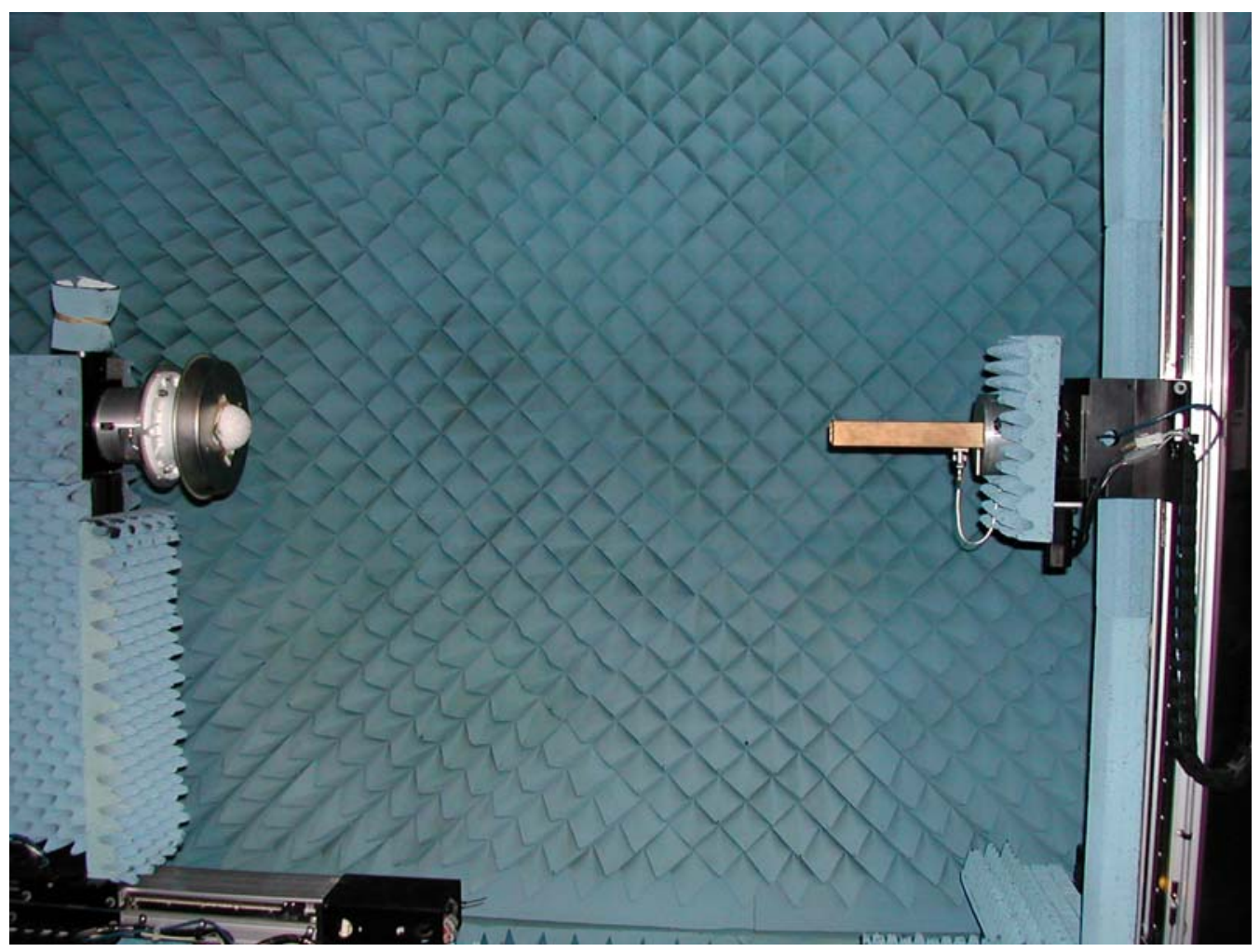

Figure 5.3: The antenna under test (left) is mounted in the anechoic chamber near an open-ended waveguide detector (right).

\subsection{Voltage Standing Wave Ratio Measurements}

The VSWR for each prototype antenna was determined using a vector network analyzer in the Time-Domain and RF Measurement Laboratory at Virginia Tech. The scattering parameter, $\mathrm{S}_{11}$, which is the same as the reflection coefficient $\Gamma$ for a one-port 
network, was measured and then converted to VSWR for the reference impedance. In this case, the reference impedance was $50 \mathrm{Ohms}$, the characteristic impedance of the coaxial cable which connected the antenna to the network analyzer.

In order to facilitate a more ideal situation for measurement, the antennas were hung from the ceiling, bore sight towards the floor, over several sections of absorbing material, as shown in Figure 5.4. This helped eliminate unwanted reflections and backscatter which could have disturbed the measurements. Data were taken over a broad range of frequencies, in small increments, and then compared to simulated data.

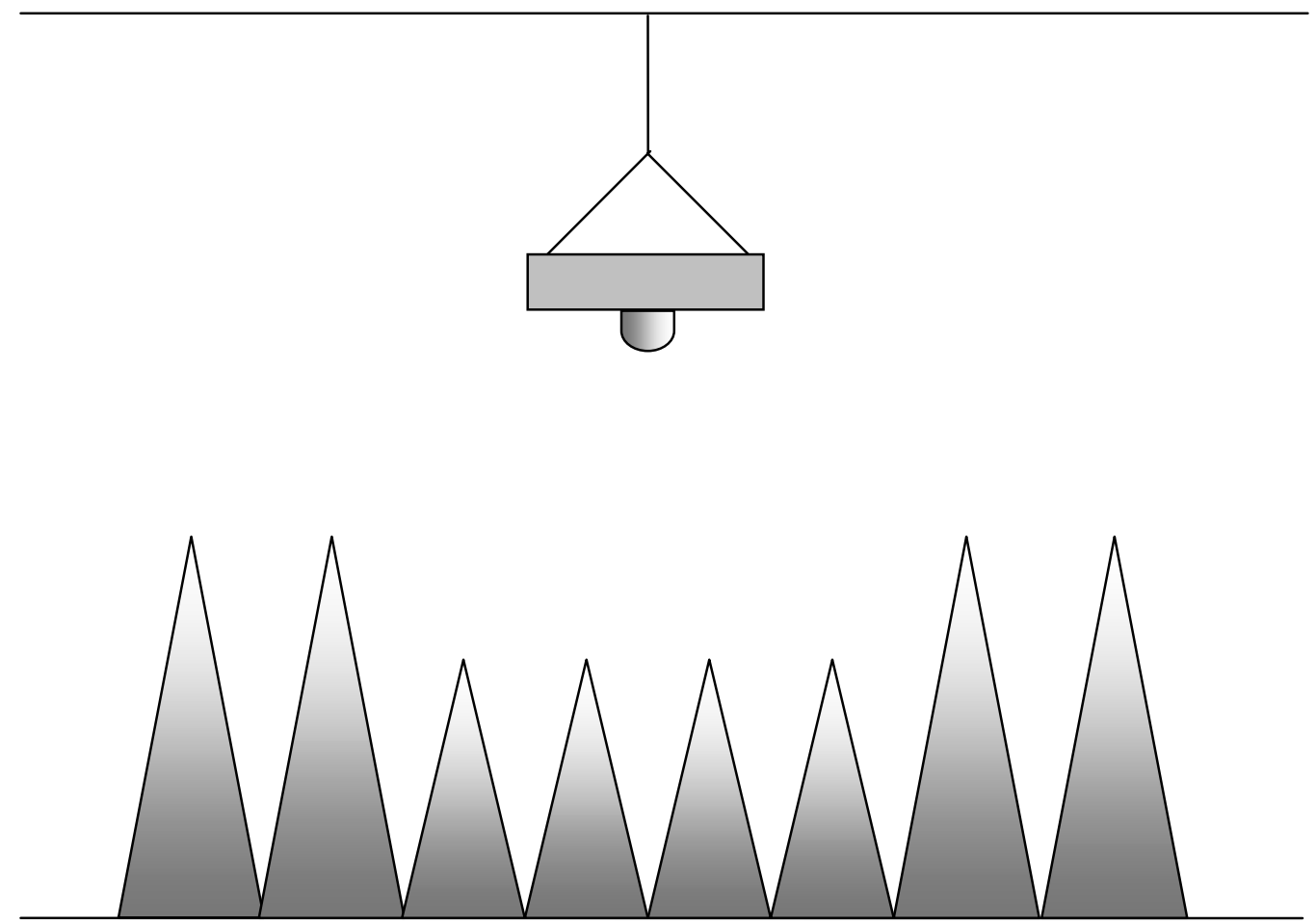

Figure 5.4: A diagram of the setup used for measuring the VSWR of the prototype antennas. The antenna was hung from the ceiling over some absorbing material similar to that used in the anechoic chamber. Diagram not to scale. 


\subsubsection{Adjustment of the Simulated Diameter of the Bifilar Hemisphere}

It was found that, by increasing the diameter size of the bifilar hemisphere model used in the simulation from the measured value of $7.4 \mathrm{~cm}$ to $7.7 \mathrm{~cm}$, excellent agreement between the measured and simulated values of the VSWR over a large range of frequencies is achieved, as seen in Figure 5.5. This is encouraging since method of moments packages are at least somewhat untrustworthy with regard to input impedance, as even slight perturbations at the feed point (such as soldering) can cause drastic variations in the measurements, especially at higher frequencies.

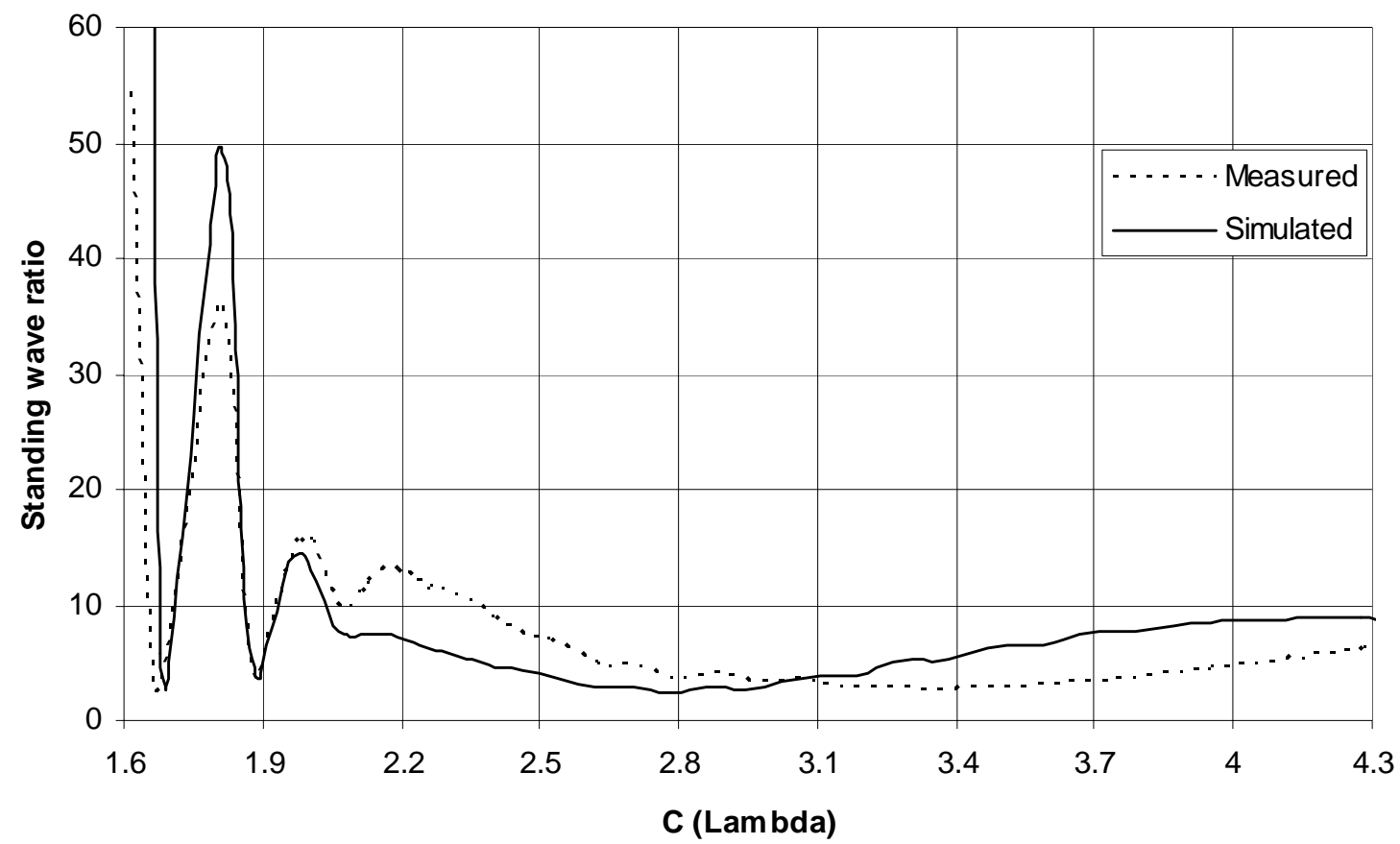

Figure 5.5: Comparison of the measured and simulated results for VSWR of the bifilar hemispherical helix.

The VSWR comparison, then, was the basis for the value of the diameter of the hemisphere chosen for simulation. It may appear, at first glance, to be an arbitrary attempt to improve data agreement, but this difference of about $4 \%$ from the measured 
diameter may be due to perturbations of the layout of the hemisphere in the prototype or to an inexact cut along the equator of the sphere, resulting in slightly more than an exact hemispherical structure. These factors could account for the slight variation in size between the constructed and simulated hemispherical helices resulting in data agreement.

\subsection{Gain Measurements for the Bifilar Hemisphere}

The total gain of the antenna over the measured range showed reasonable agreement with the simulated results. Figure 5.6 depicts these results. The measured value was remarkably constant over the entire range of measured frequencies, varying less than approximately $0.7 \mathrm{~dB}$. It is noteworthy that the gain in the H-plane showed much better agreement than the gain in the E-plane, as seen in Figures 5.7 and 5.8.

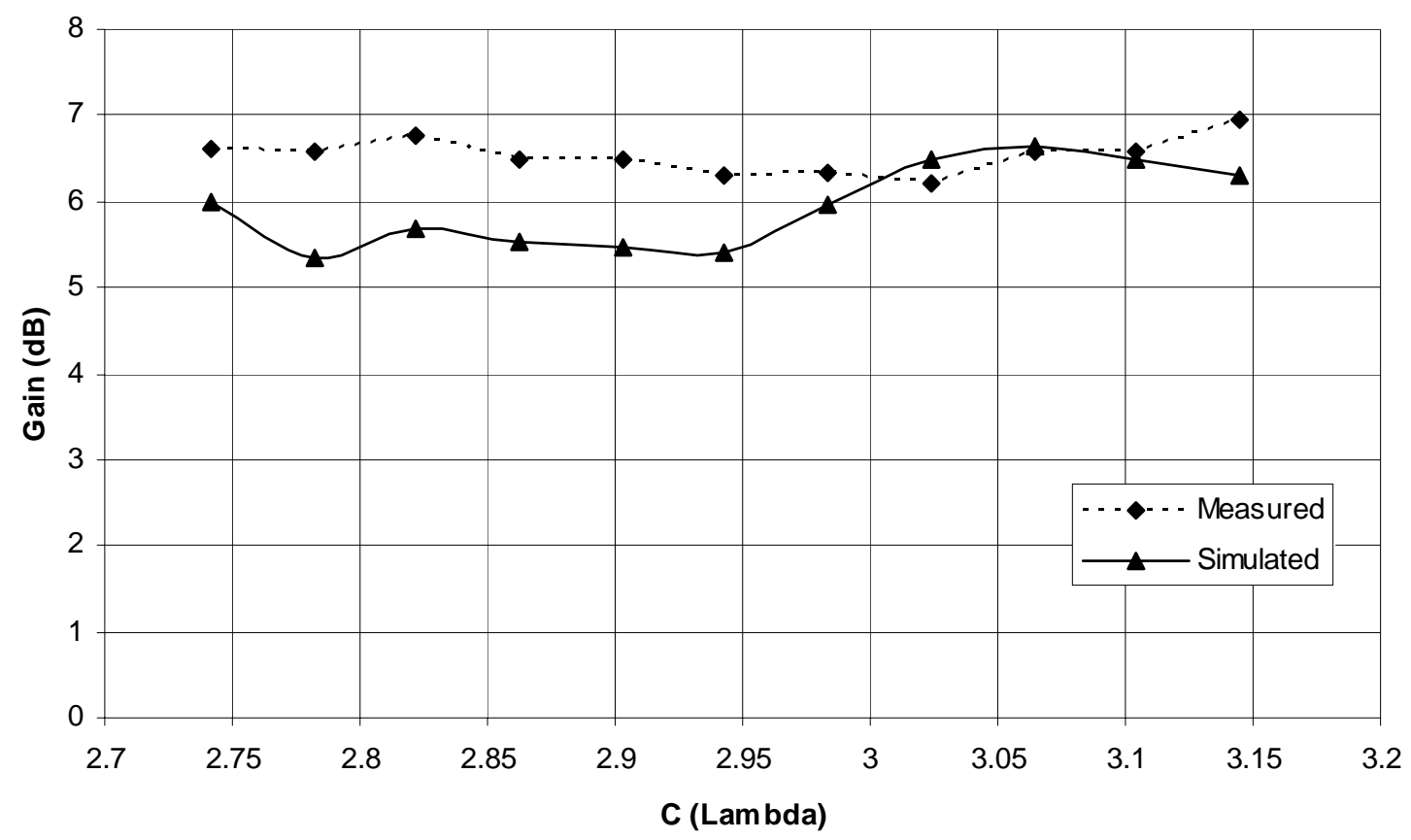

Figure 5.6: Comparison of the measured (dashed) and simulated (solid) results for the maximum gain of the antenna. 


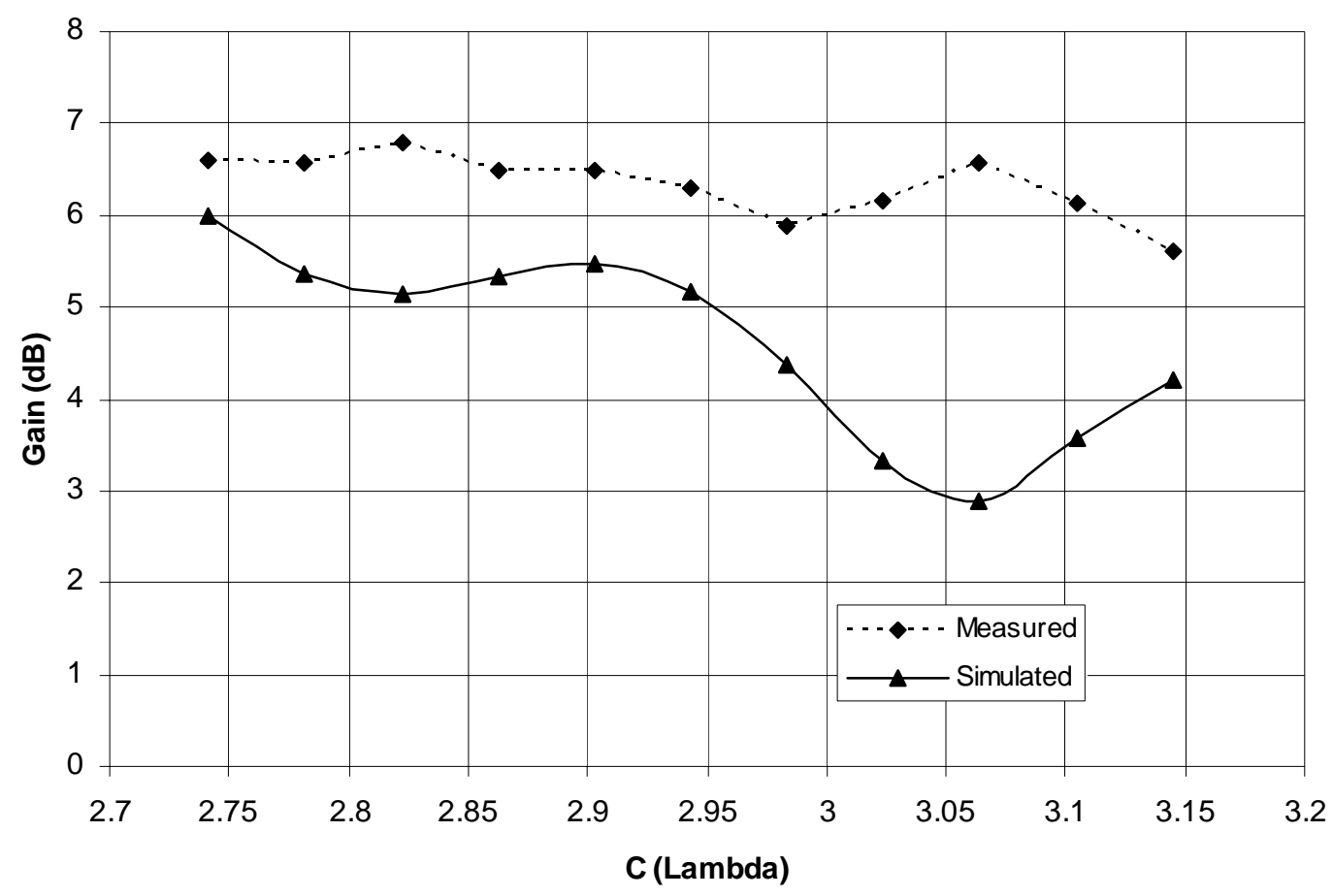

Figure 5.7: E-plane maximum gain comparison for measured (dashed) and simulated (solid) results.

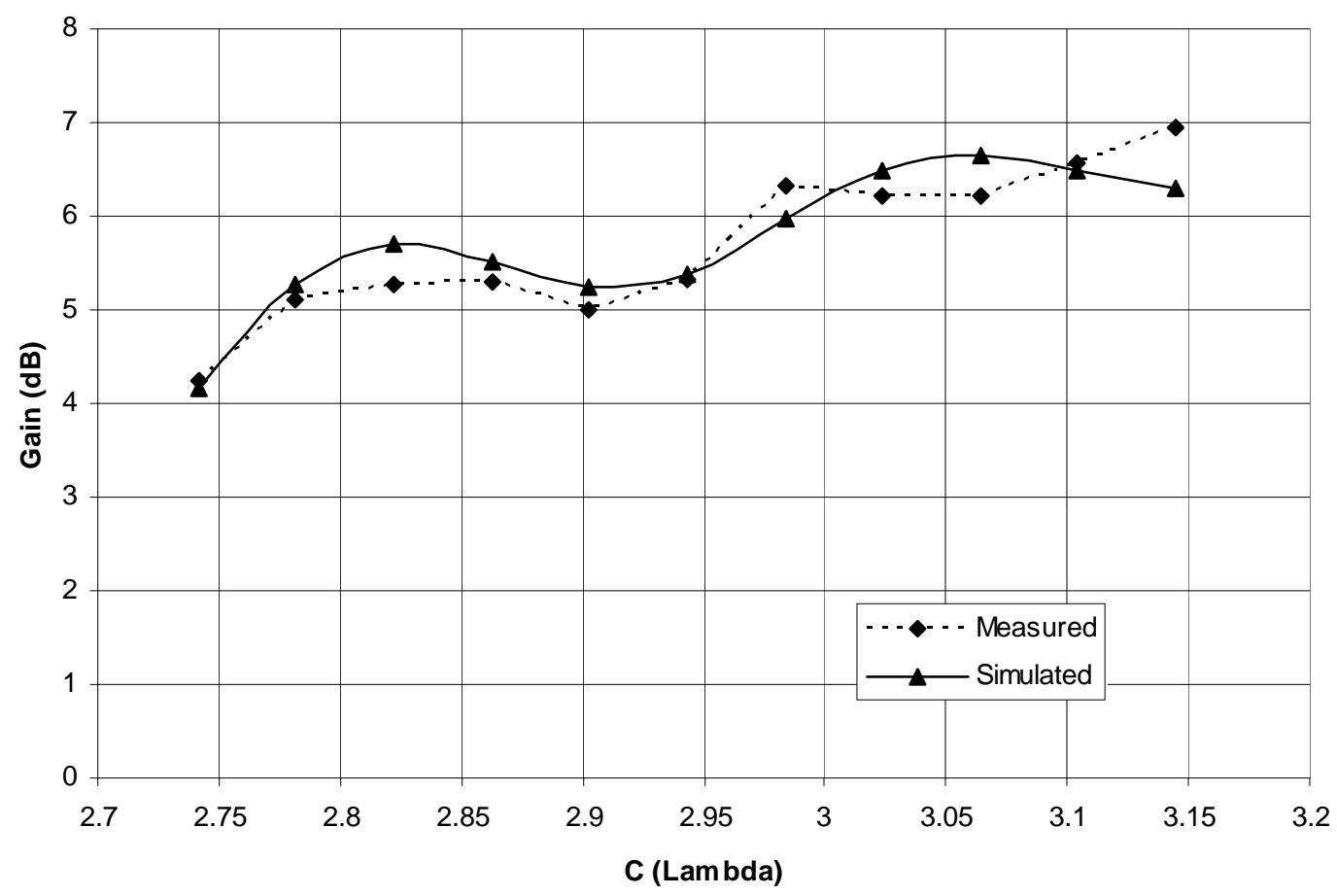

Figure 5.8: H-plane maximum gain comparison for measured (dashed) and simulated (solid) results. 


\subsubsection{Pattern Measurements}

The patterns, depicted in Figure 5.9, show a very good agreement between measured and simulated data. The null at bore sight is distinctly pronounced in the measured data, as predicted, however there is in fact some radiation in the axial direction. This is likely due to imperfections and asymmetries in the prototype which result in less than perfect destructive interference along the axis. This may be beneficial, however, if some limited amount of radiation in the axial direction is required; in a more controlled situation, a pre-planned asymmetry may be introduced into the antenna design to prevent total suppression of the radiation at bore sight. Appendix A shows all the measured patterns along with the simulated patterns for comparison purposes.

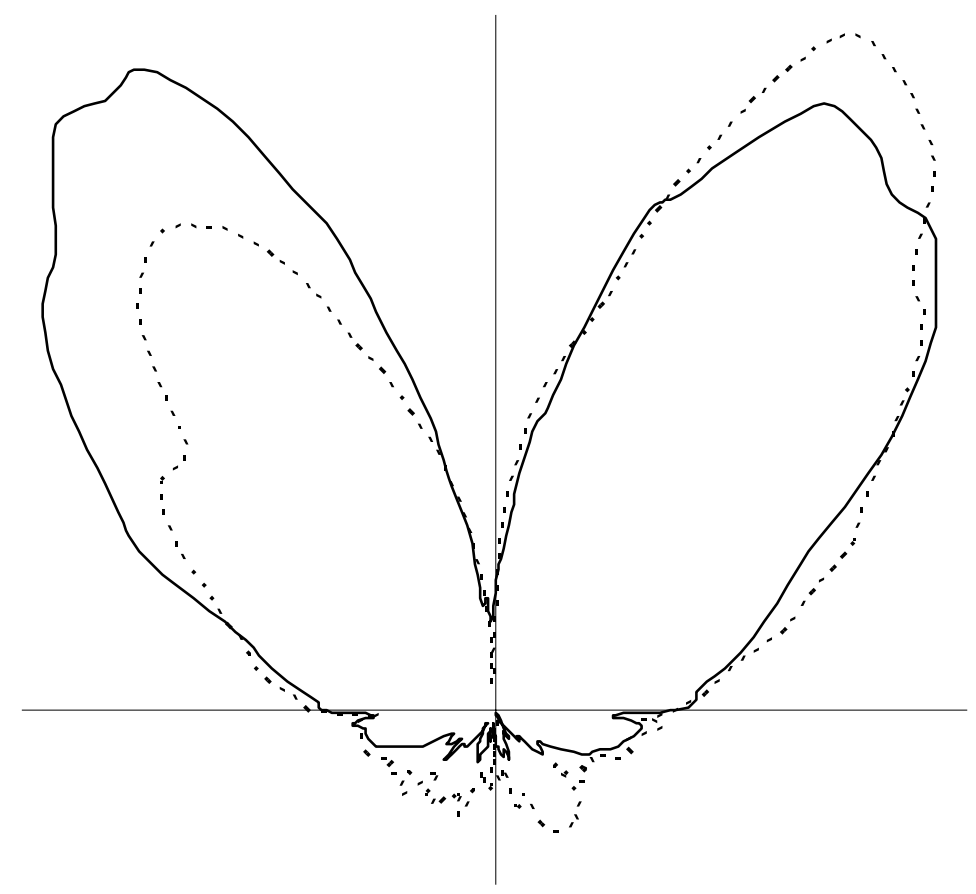

Figure 5.9: The measured radiation pattern in the E-plane for the bifilar hemispherical helix at a normalized circumference of $2.86 \lambda$. Compare the simulated results of Figure 4.6. Shown are the $\theta$ (dashed) and $\varphi$ (solid) components of the radiated field. 
The bifilar hemispherical helix, similar to the volute antenna described earlier, appears to have a very narrow bandwidth of only a few percent. The polarization is, in general, elliptical with only very small beamwidths and bandwidths being nearly circularly polarized with axial ratios of less than $3 \mathrm{~dB}$ and phase differences within about $15-20^{\circ}$ of $90^{\circ}$. The gain of the antenna and the reasonably low VSWR were measured to have a bandwidth of at least 13 to $14 \%$. Additionally, although there is some variation between data in the E-plane and H-plane, this antenna has proved to be fairly omnidirectional. Asymmetries would appear to result from the fact that this is only a bifilar hemispherical helix. A quadrifilar configuration shows more symmetry and thus would likely show more symmetry between E- and H-plane patterns. These results seem to indicate that, if further optimized, the bifilar hemispherical helix may be a competitor with the resonant quadrifilar helix when a conical, or bifurcated, beam is required. The primary advantage of the bifilar hemispherical helix is that it does not require a bulky phase shifting device as does the volute antenna. Currently, however, the bifilar hemispherical helix lacks the broad beamwidth with circular polarization that the volute has. 

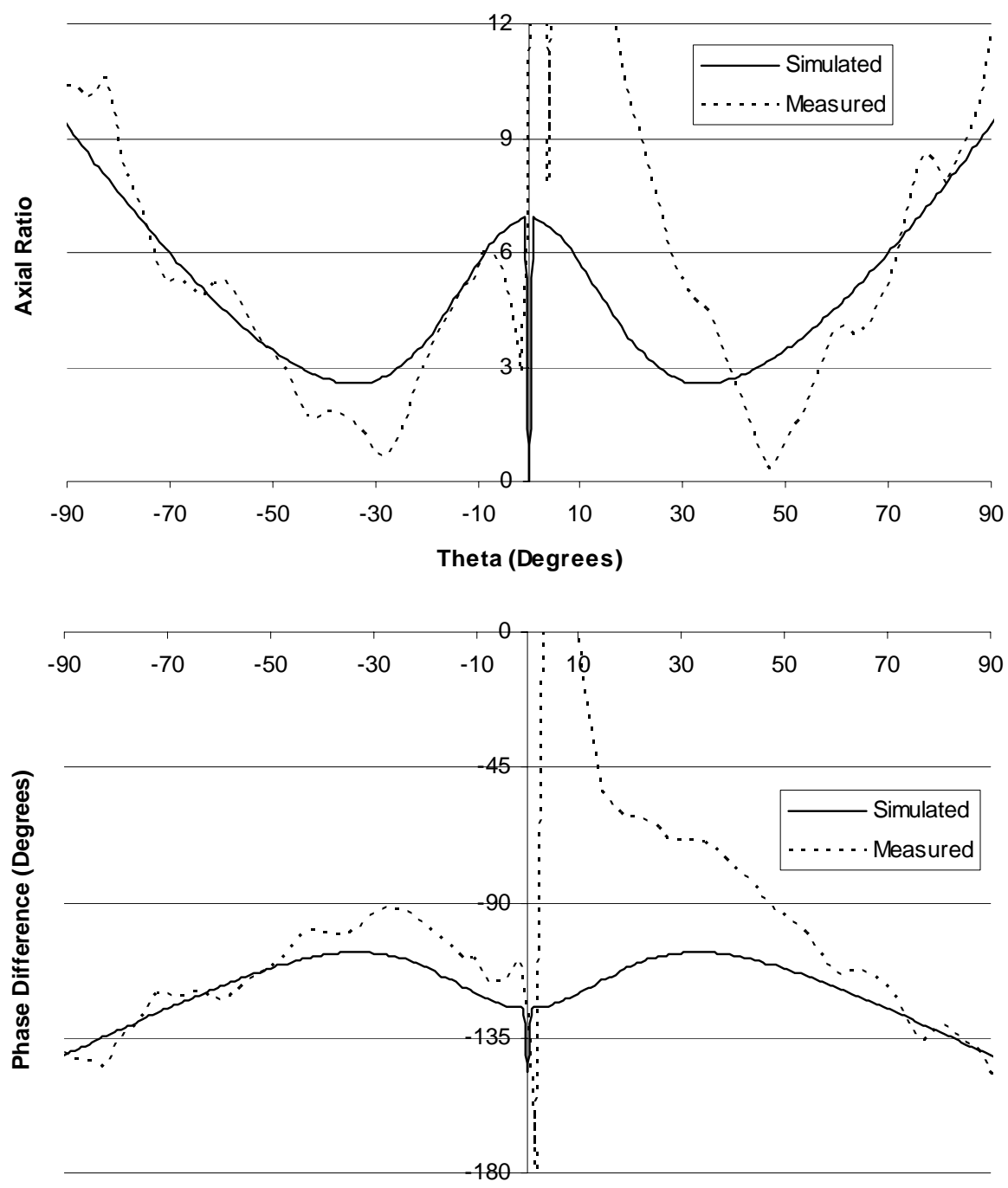

Figure 5.10: Comparison plots of the axial ratio (top) and phase difference (bottom) in the H-plane for the bifilar hemispherical helix at a normalized circumference of $2.90 \lambda$. Shown are the measured (dashed) and simulated (solid) results.

\subsection{Measurements for the Quadrifilar Hemisphere}

The quadrifilar helical antenna measurements were limited by the poor VSWR, which makes the antenna a poor radiator (Figure 5.11). As a result, although other radiation characteristics may be useful, the antenna itself is not, unless a complicated matching network is also utilized. Additionally, it is seen in the results that the VSWR is 
oscillatory, perhaps accounting for the difficulty NEC-4 had with producing accurate results. In such a case, very slight variations in the feed region can cause drastic differences in the VSWR. NEC-4 seemed successful, however, in producing a waveform that oscillated and began to level out at the high end of the range.

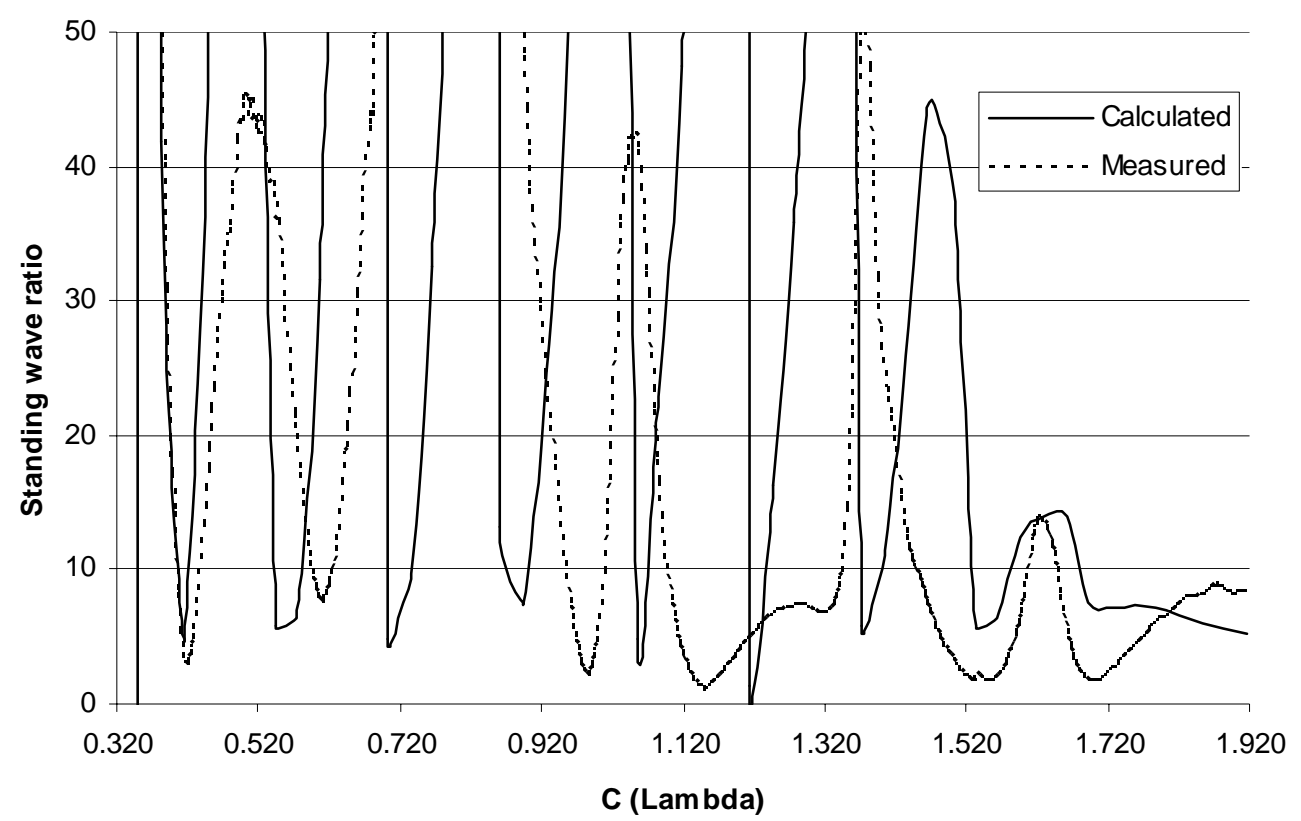

Figure 5.11: A comparison of the measured (dashed) and simulated (solid) values of the VSWR for the quadrifilar hemispherical helix.

\subsubsection{Pattern Measurements}

It is noteworthy that the patterns, axial ratio and phase difference results from the measurements are very close to the simulated values. An example is shown in Figures 5.12 and 5.13. The primary difference here is in the phase difference, where a $180^{\circ} \mathrm{shift}$ between the simulated and measured values is seen. Since this phase shift appears in all the phase difference measurements in the E-plane, it may be assumed that it is simply the 
result of a change of convention in the measurement apparatus contrary to the simulations.
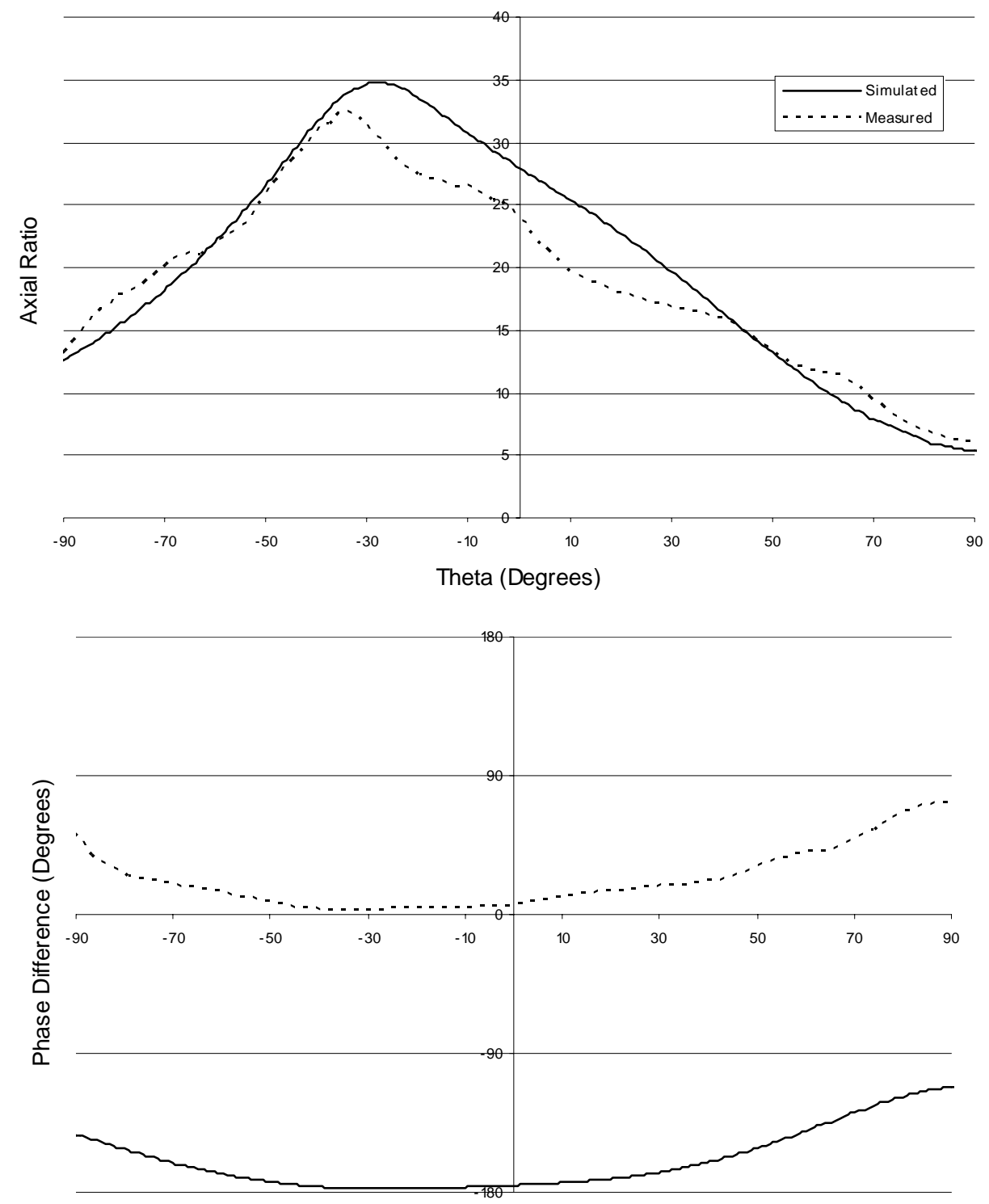

Figure 5.12: Comparison plots of the axial ratio (top) and phase difference (bottom) for the quadrifilar hemispherical helix. Shown are the measured (dashed) and simulated (solid) results. 


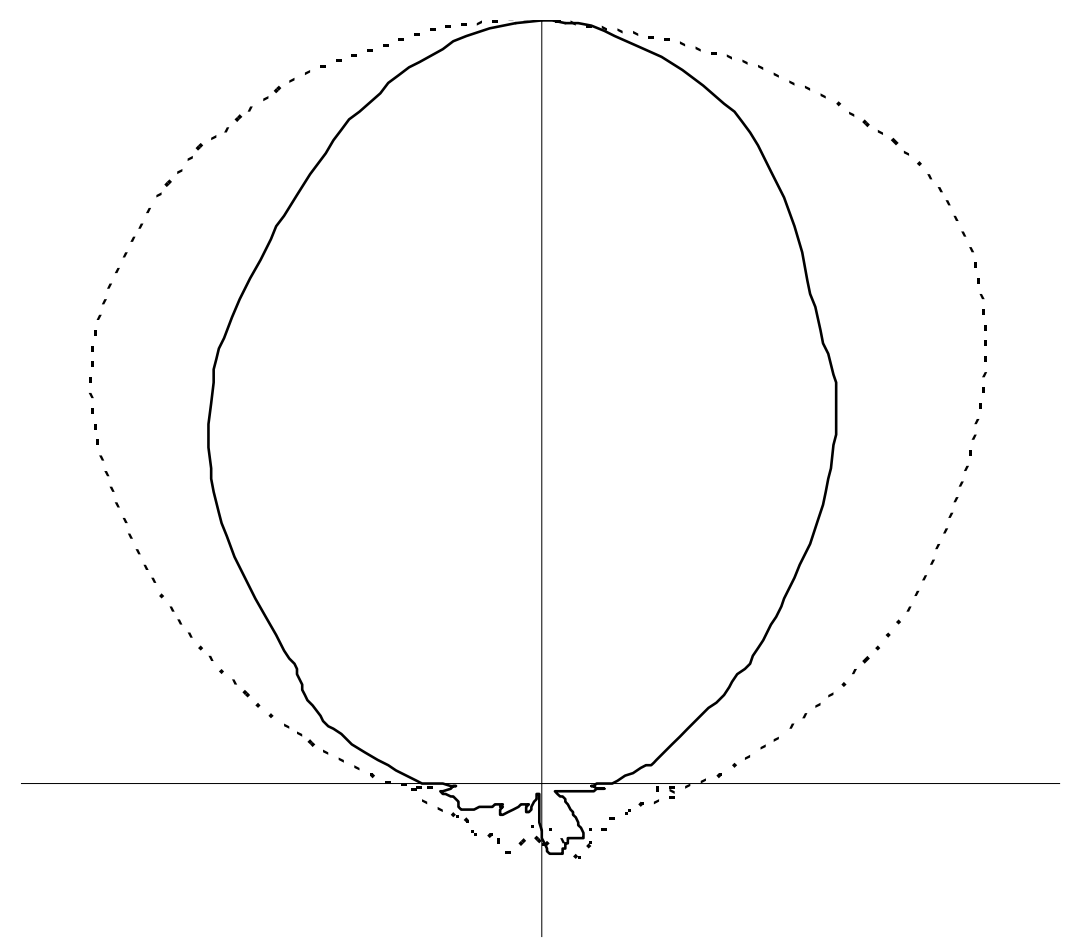

Figure 5.13: The measured radiation pattern for the quadrifilar hemispherical helix at a normalized circumference of $0.80 \lambda$. The $\theta$ (dashed) and $\varphi$ (solid) components of the field are shown. Compare Figure 4.11 for simulated results. 


\section{Chapter 6. Conclusions and Suggestions for Further Work}

The top-fed bifilar hemispherical helix has been shown to have some useful qualities over approximately a $14 \%$ bandwidth, including operation purely in the axialnull mode, a very flat gain curve (less than $0.8 \mathrm{~dB}$ variation) and a moderately low VSWR of about 3.5 with a variation of less than 0.5 . The antenna produced modest circular polarization over small beamwidths and a limited bandwidth on either side of bore sight and elliptical polarization in general. The geometry of the hemisphere allows for a much more stable and compact version of the spherical helix, and thus an attractive alternative from a mechanical perspective.

Further investigation of this antenna could prove beneficial. One factor that requires further examination is the radius of conductor, as a thicker conductor would likely improve impedance characteristics and possibly radiation characteristics (such as polarization). It may also prove that slight rotation of one of the arms about the axis could control the depth of the null at bore sight, and may even improve the characteristics of the antenna.

The side-fed quadrifilar hemispherical helix has proven to be less promising than the previous design, owing primarily to its very high (oscillatory) VSWR. As a result, the antenna would seem to provide no new improvements over existing designs. It may turn out, however, that by increasing the conductor diameter there could be some improvement in the VSWR; however, it is expected that the oscillatory nature of this parameter over a range of frequencies would remain, thus maintaining the difficulty. 


\section{References}

[1] G. J. Burke, A. J. Poggio, Numerical Electromagnetics Code (NEC) - Method of Moments, UCID 18834, Lawrence Livermore Laboratory, 1981.

[2] J. D. Kraus, Antennas. McGraw-Hill: New York, 1950.

[3] W. L. Stutzman and G. A. Thiele, Antenna Theory and Design, Second Edition. John Wiley \& Sons, Inc.: New York, 1998.

[4] H.E. King and J.L. Wong, "Characteristics of 1 to 8 Wavelength uniform helical antennas," IEEE Transactions on Antennas and Propagation, Vol. AP-28 No. 2. March, 1980.

[5] C. A. Balanis, Advanced Engineering Electromagnetics, John Wiley \& Sons, Inc., New York, 1989.

[6] A. Safaai-Jazi, "New scheme for determining polarization of electromagnetic waves," Virginia Tech.

[7] J. C. Cardoso, "The spherical helical antenna," Master's Thesis, Virginia Tech, 1992.

[8] R. L. Lovestead, "Helical antenna optimization using genetic algorithms," Master's Thesis, Virginia Tech, 1999.

[9] J. C. Cardoso and A. Safaai-Jazi, "Radiation characteristics of a spherical helical antenna," IEE Proceedings - Microwaves, Antennas and Propagation, Vol. 143, No. 1. February, 1996.

[10] J. C. Cardoso and A. Safaai-Jazi, "Spherical helical antenna with circular polarisation over a broad beam," Electronics Letters, Vol. 29, No. 4. February 18, 1993.

[11] S. H. Zainud-Deen, N. A. M. Salem, S. M. M. Ibrahem and H. A. Motaafy, "Investigation of an octifilar helix antenna," Nineteenth National Radio Science Conference, Alexandria, March 19-21, 2002.

[12] C. C. Kilgus, "Resonant quadrifilar helix," IEEE Transactions on Antennas and Propagation, Vol. 17, No. 3, May, 1969. 
[13] R. C. Johnson, Ed., Antenna Engineering Handbook,3rd ed., McGraw-Hill, New York, 1993.

[14] J. M. Tranquilla and S. R. Best, "A study of the quadrifilar helix antenna for Global Positioning System (GPS) applications," IEEE Transactions on Antennas and Propagation, Vol. 38, No. 10, October 1990.

[15] A. T. Adams, R. K. Greenough, R. F. Wallenberg, A. Mendelovicz and C. Lumjiak, "The quadrifilar helix antenna," IEEE Transactions on Antennas and Propagation, Vol. AP-22, No. 2, March 1974.

[16] C. C. Kilgus, "Shaped-conical radiation pattern performance of the backfire quadrifilar helix," IEEE Transactions of Antennas and Propagation, Vol. 23, No. 3, May, 1975.

[17] H. Kawakami, Y. Iitsuka, S. Kogiso, S. Kon and G. Sato, "Design of variable turn bifilar helical antenna for mobile communication," IEE 10th International Conference on Antennas and Propagation, Conference Publication No. 436, April 14-17, 1997.

[18] E. Weeratumanoon and A. Safaai-Jazi, "Truncated spherical helical antennas," Electronics Letters, Vol. 36, No. 7, March 30, 2000.

[19] E. Weeratumanoon, "Helical antennas with truncated spherical geometry," Master's Thesis, Virginia Tech, 2000.

[20] K. Y. Chan, H. T. Hui and E. K. N. Yung, "Central-fed hemispherical helical antenna," IEEE, 2000.

[21] H.T. Hui, K.Y. Chan, E.K.H. Yung, X.Q. Shing, "Coaxial-feed axial mode hemispherical helical antenna," Electronics Letters, Vol. 35, No. 23, November 11, 1999.

[22] S. R. Best, "The performance properties of an electrically small folded spherical helix antenna," IEEE, 2000. 


\section{Appendix A. Bifilar Hemispherical Helix Simulated Radiation Patterns}

Both the simulated and measured radiation patterns for the bifilar hemispherical helical antenna are herein shown in their entirety. Figures A.1 through A.11 are E-plane

data, and Figures A.12 through A.22 are H-plane data. All pattern plots are shown using a linear scale to facilitate a more visually descriptive rendering of the results which may sometimes be impeded by the use of logarithmic plots. Also included for each pattern is the axial ratio (in $\mathrm{dB}$ ) and phase difference (in degrees) over the region of space above the ground plane. 

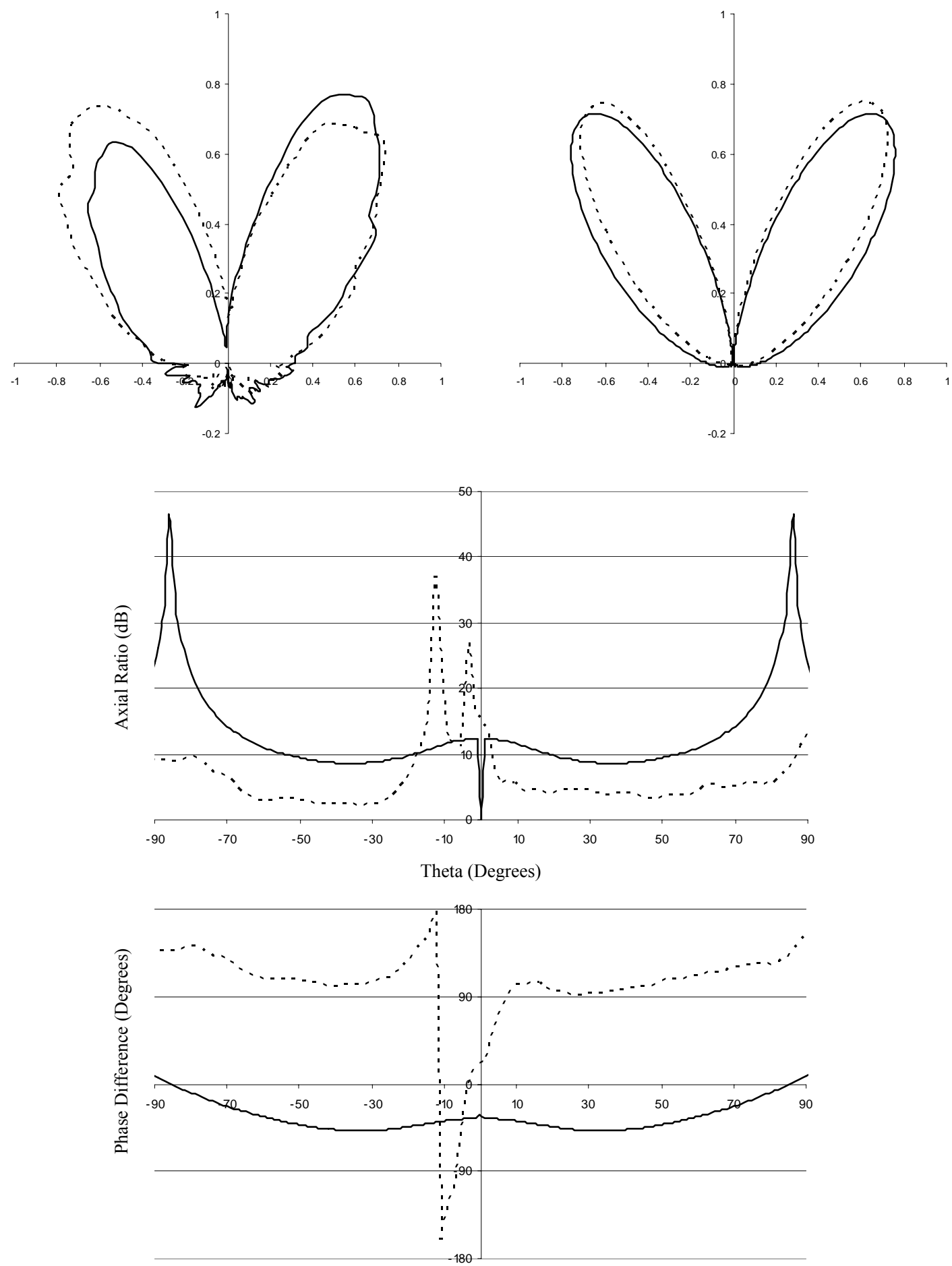

Figure A.1: The measured (top left) and simulated (top right) patterns, with the theta (dashed) and phi (solid) components, and the corresponding axial ratio (middle, in $\mathrm{dB}$ ) and phase difference (bottom, in degrees) plots with the measured (dashed) and simulated (solid) results at a frequency of $3.40 \mathrm{GHz}$ (normalized circumference of $2.74 \lambda$ ). 

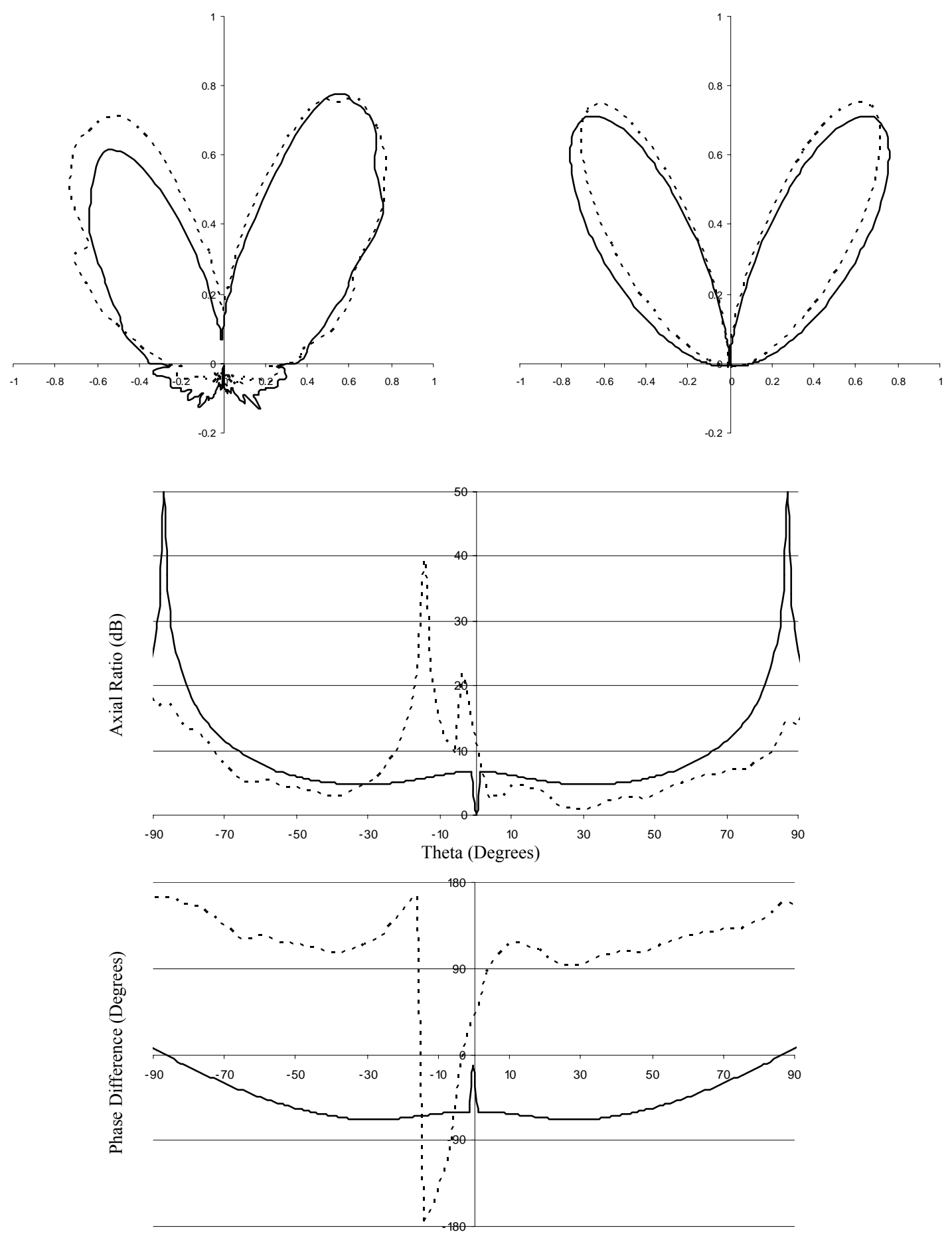

Figure A.2: The measured (top left) and simulated (top right) patterns, with the theta (dashed) and phi (solid) components, and the corresponding axial ratio (middle, in $\mathrm{dB}$ ) and phase difference (bottom, in degrees) plots with the measured (dashed) and simulated (solid) results at a frequency of $3.45 \mathrm{GHz}$ (normalized circumference of $2.78 \lambda$ ). 

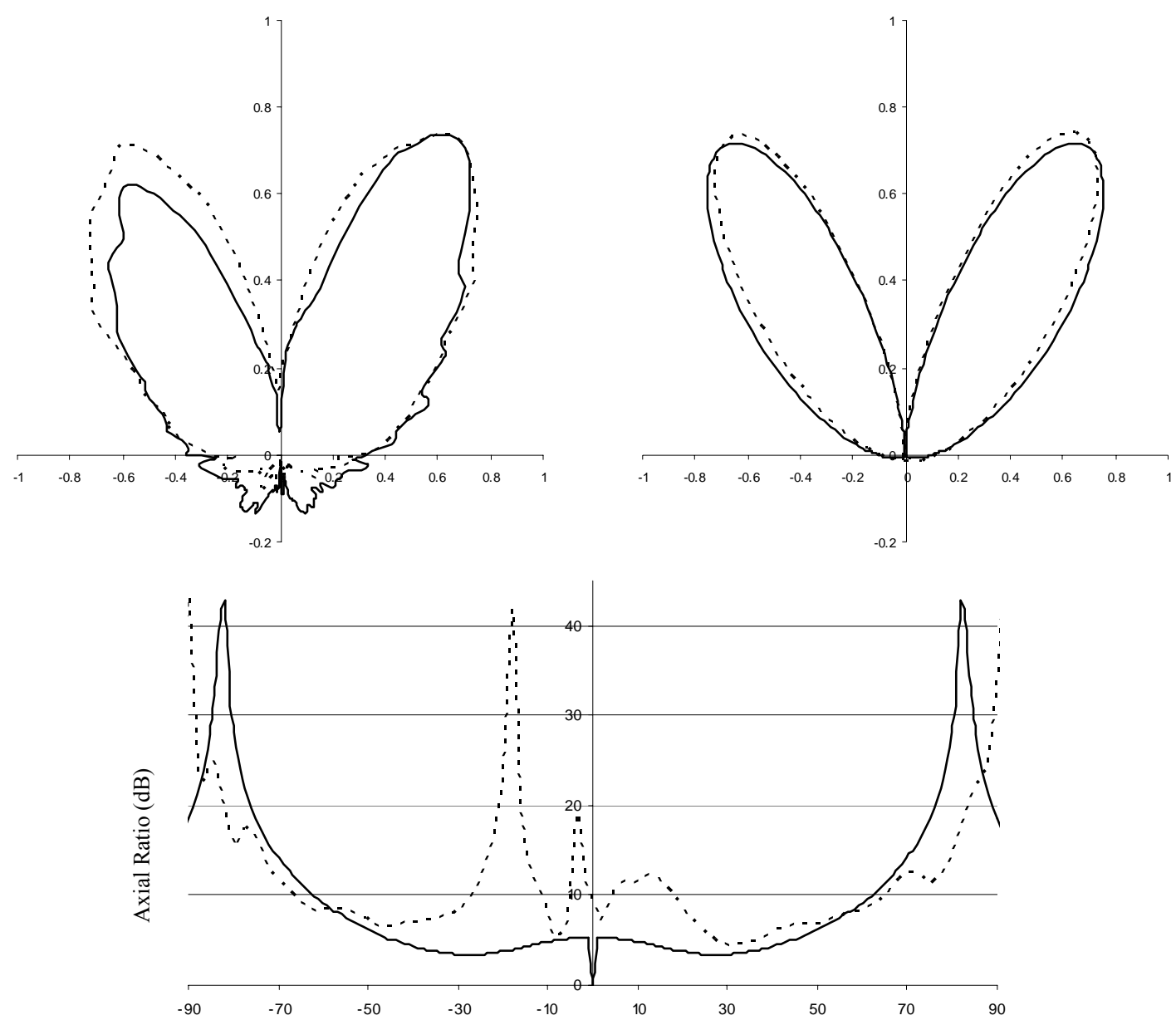

Theta (Degrees)

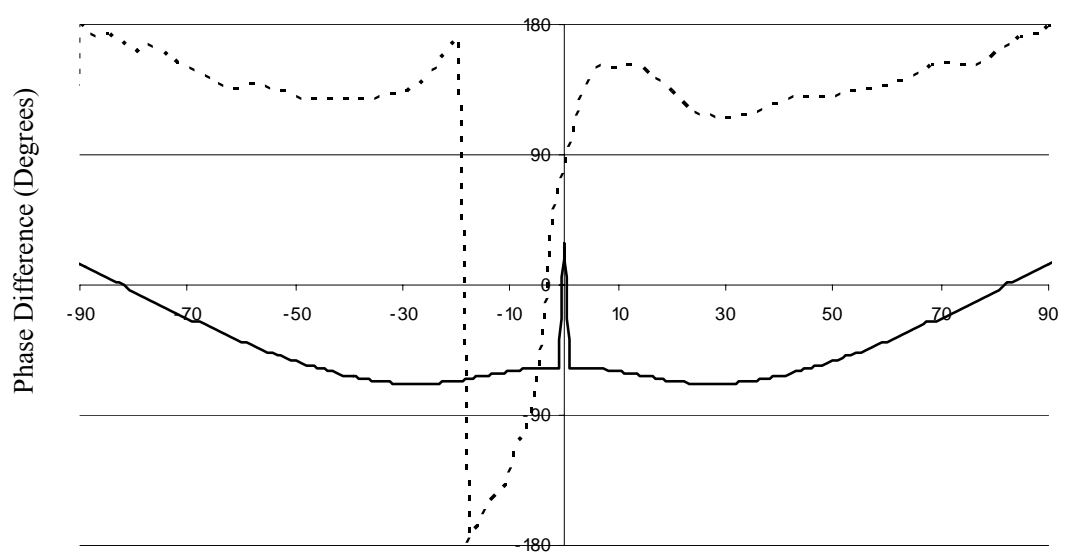

Figure A.3: The measured (top left) and simulated (top right) patterns, with the theta (dashed) and phi (solid) components, and the corresponding axial ratio (middle, in $\mathrm{dB}$ ) and phase difference (bottom, in degrees) plots with the measured (dashed) and simulated (solid) results at a frequency of $3.50 \mathrm{GHz}$ (normalized circumference of $2.82 \lambda$ ). 

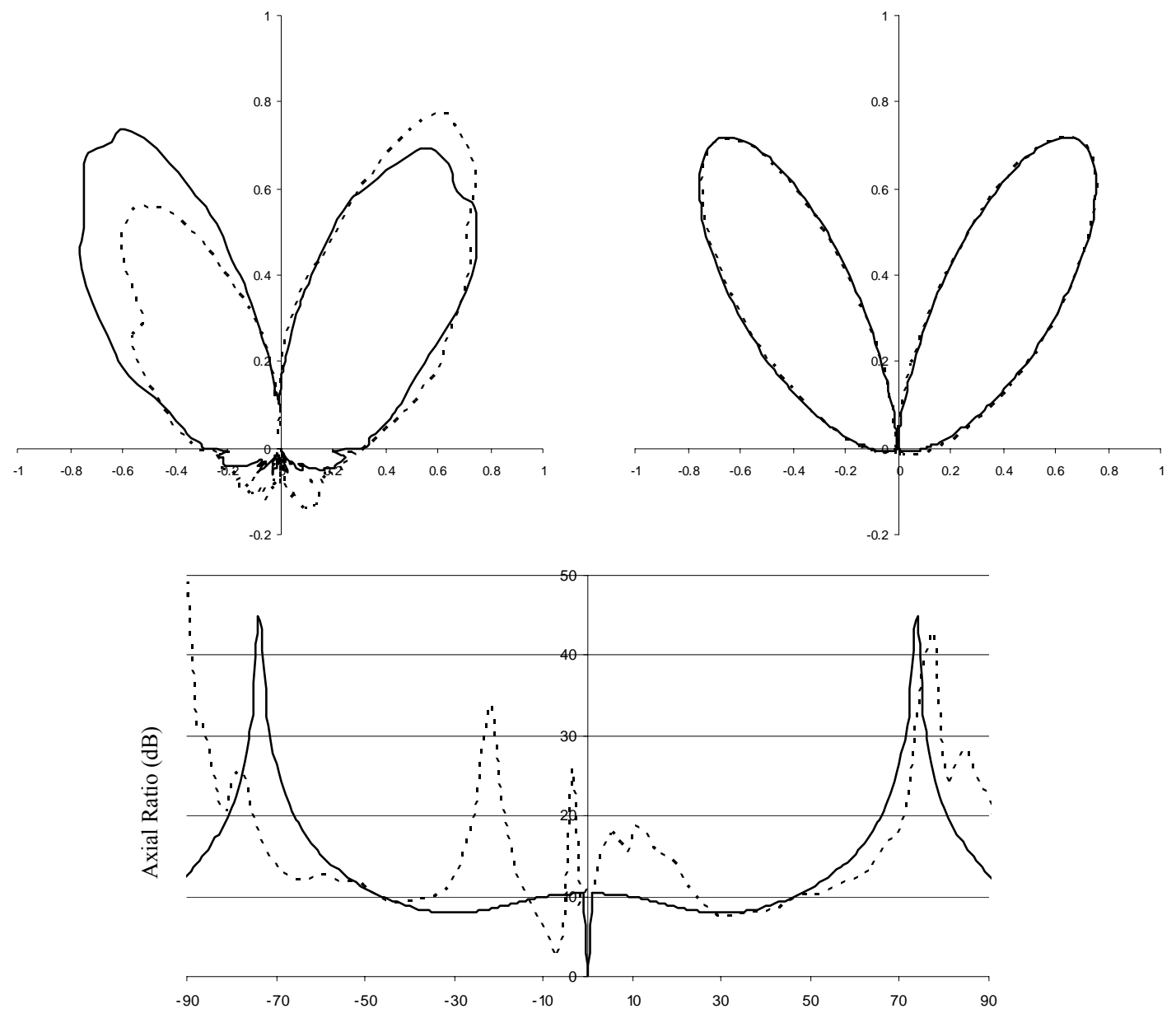

Theta (Degrees)

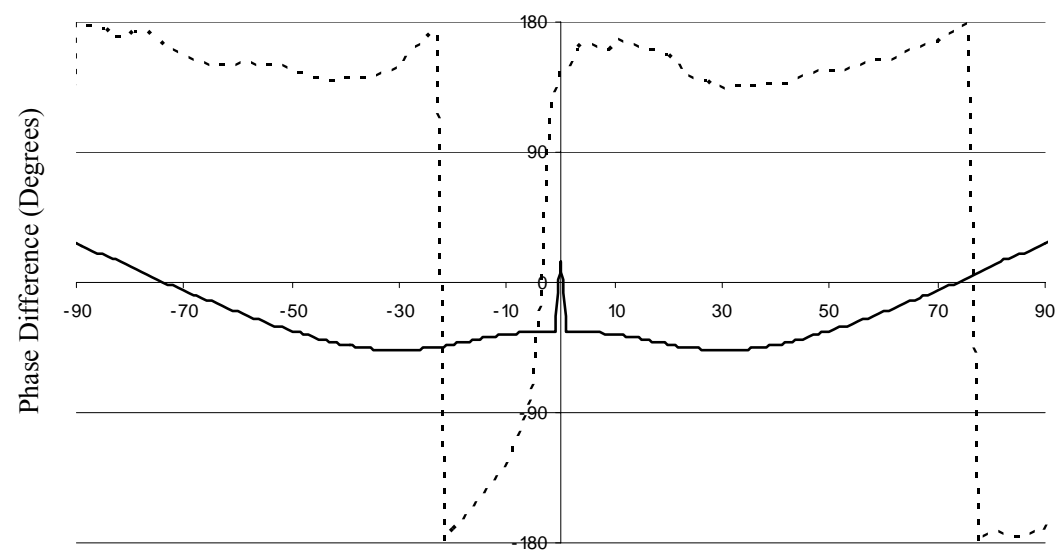

Figure A.4: The measured (top left) and simulated (top right) patterns, with the theta (dashed) and phi (solid) components, and the corresponding axial ratio (middle, in $\mathrm{dB}$ ) and phase difference (bottom, in degrees) plots with the measured (dashed) and simulated (solid) results at a frequency of $3.55 \mathrm{GHz}$ (normalized circumference of $2.86 \lambda$ ). 

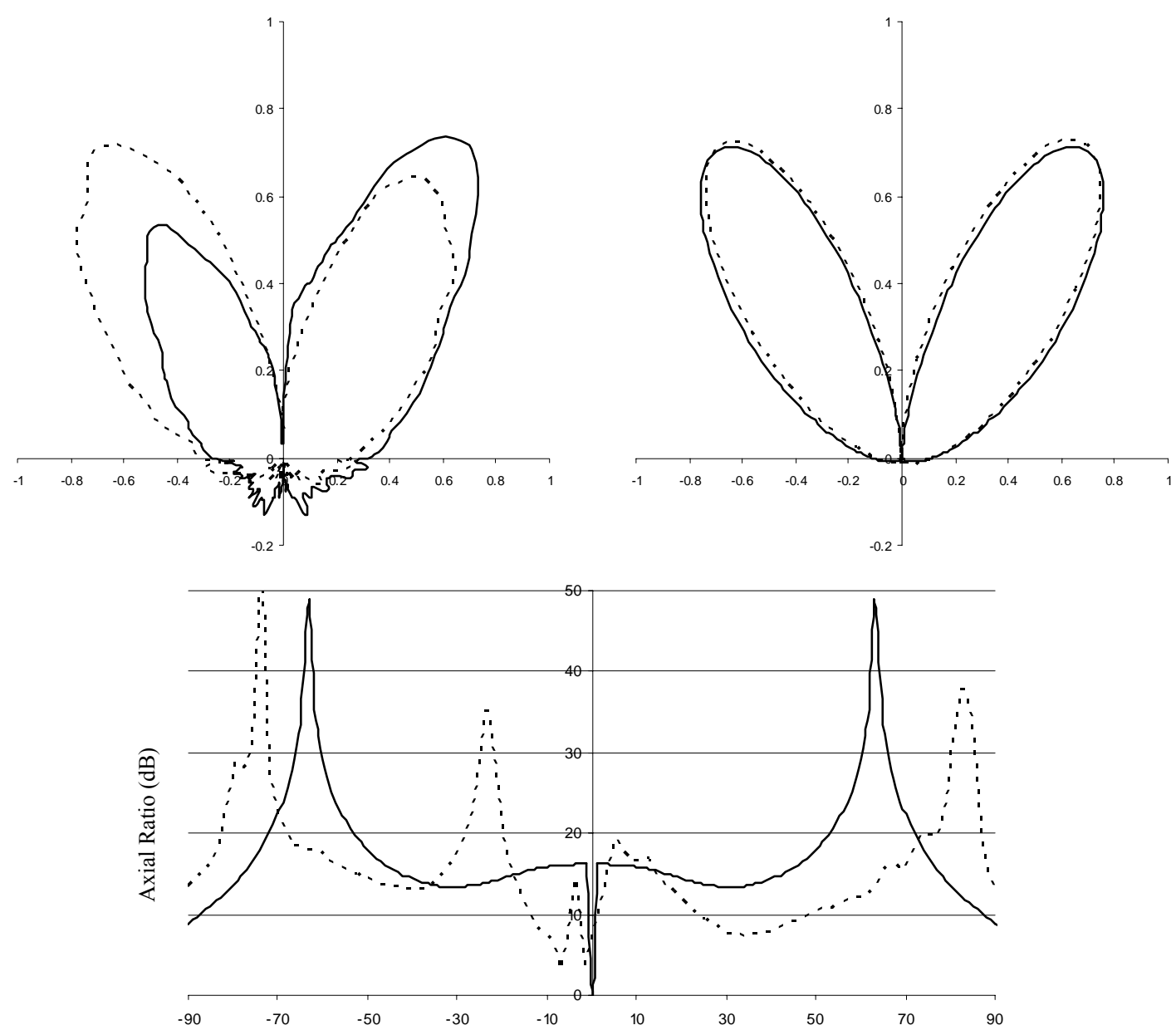

Theta (Degrees)

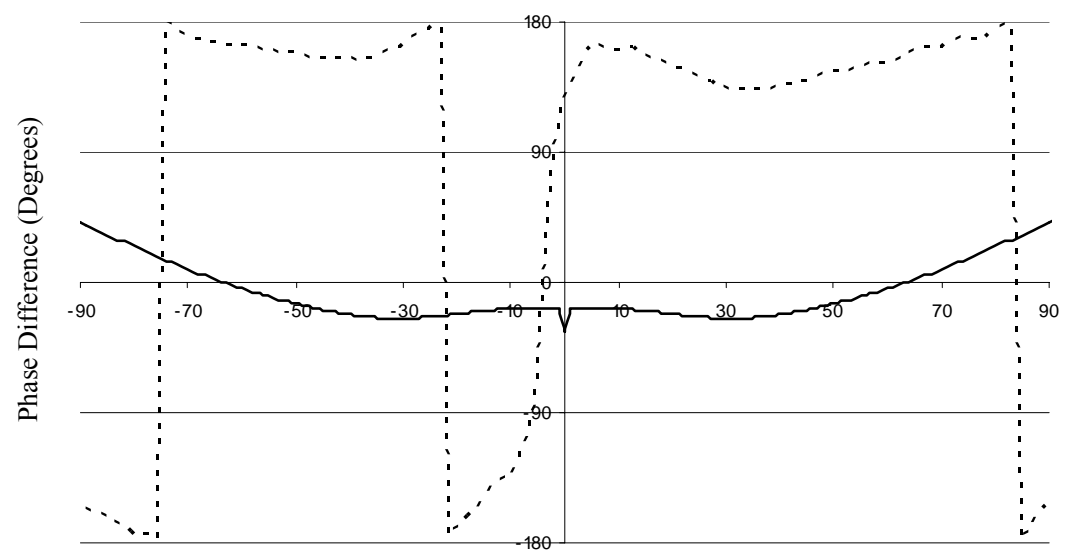

Figure A.5: The measured (top left) and simulated (top right) patterns, with the theta (dashed) and phi (solid) components, and the corresponding axial ratio (middle, in $\mathrm{dB}$ ) and phase difference (bottom, in degrees) plots with the measured (dashed) and simulated (solid) results at a frequency of $3.60 \mathrm{GHz}$ (normalized circumference of $2.90 \lambda$ ). 

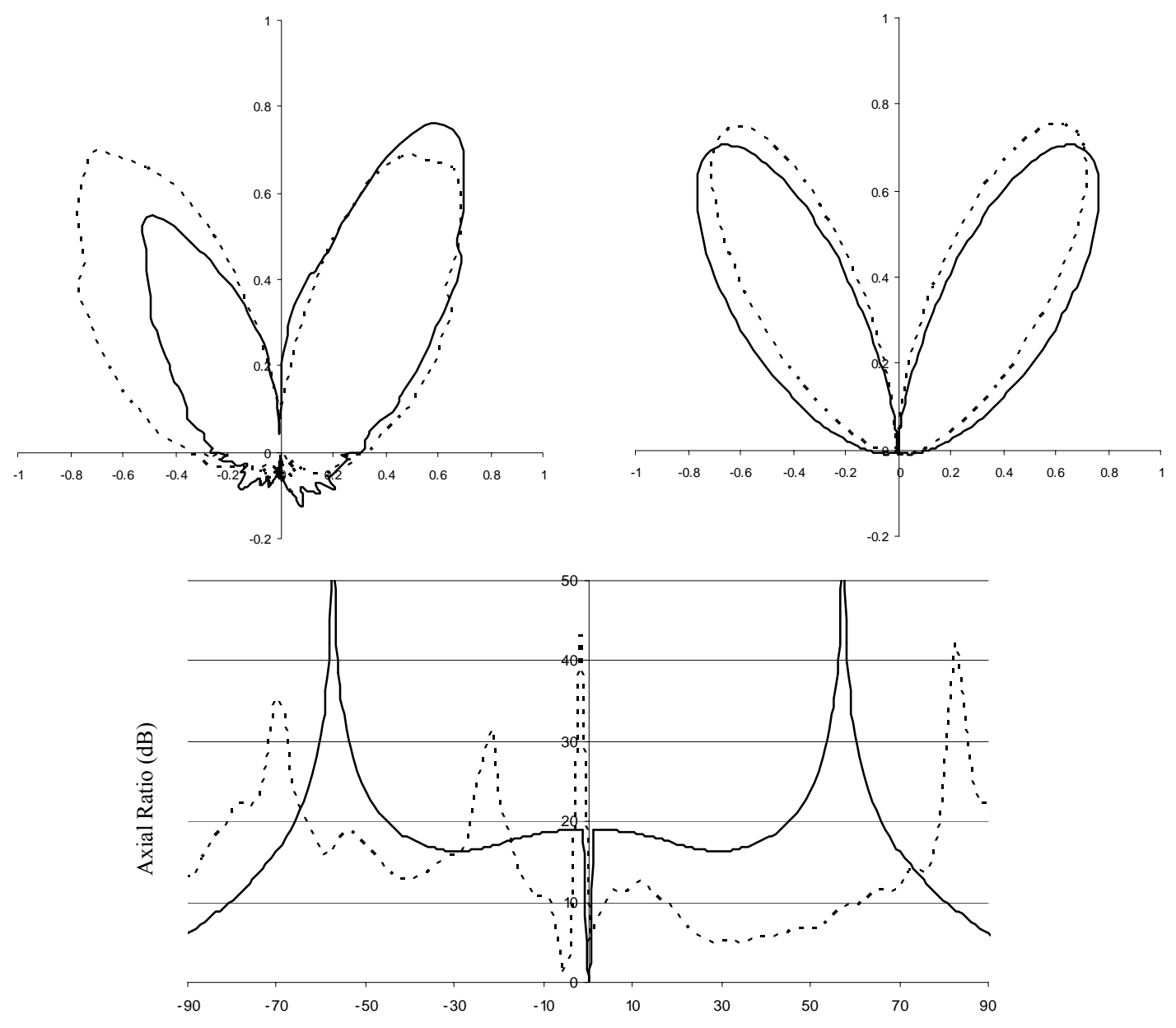

Theta (Degrees)

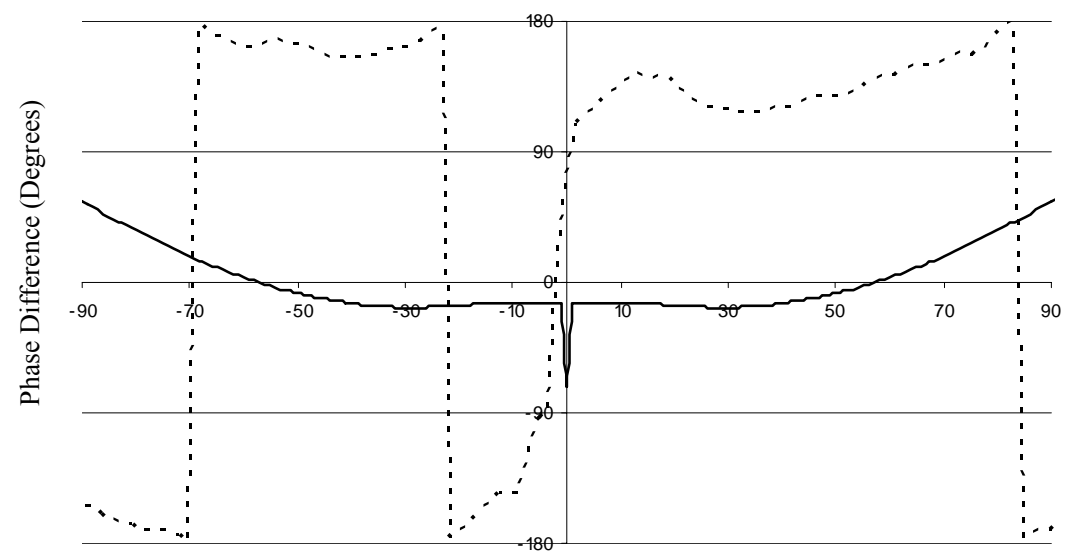

Figure A.6: The measured (top left) and simulated (top right) patterns, with the theta (dashed) and phi (solid) components, and the corresponding axial ratio (middle, in $\mathrm{dB}$ ) and phase difference (bottom, in degrees) plots with the measured (dashed) and simulated (solid) results at a frequency of $3.65 \mathrm{GHz}$ (normalized circumference of $2.94 \lambda$ ). 

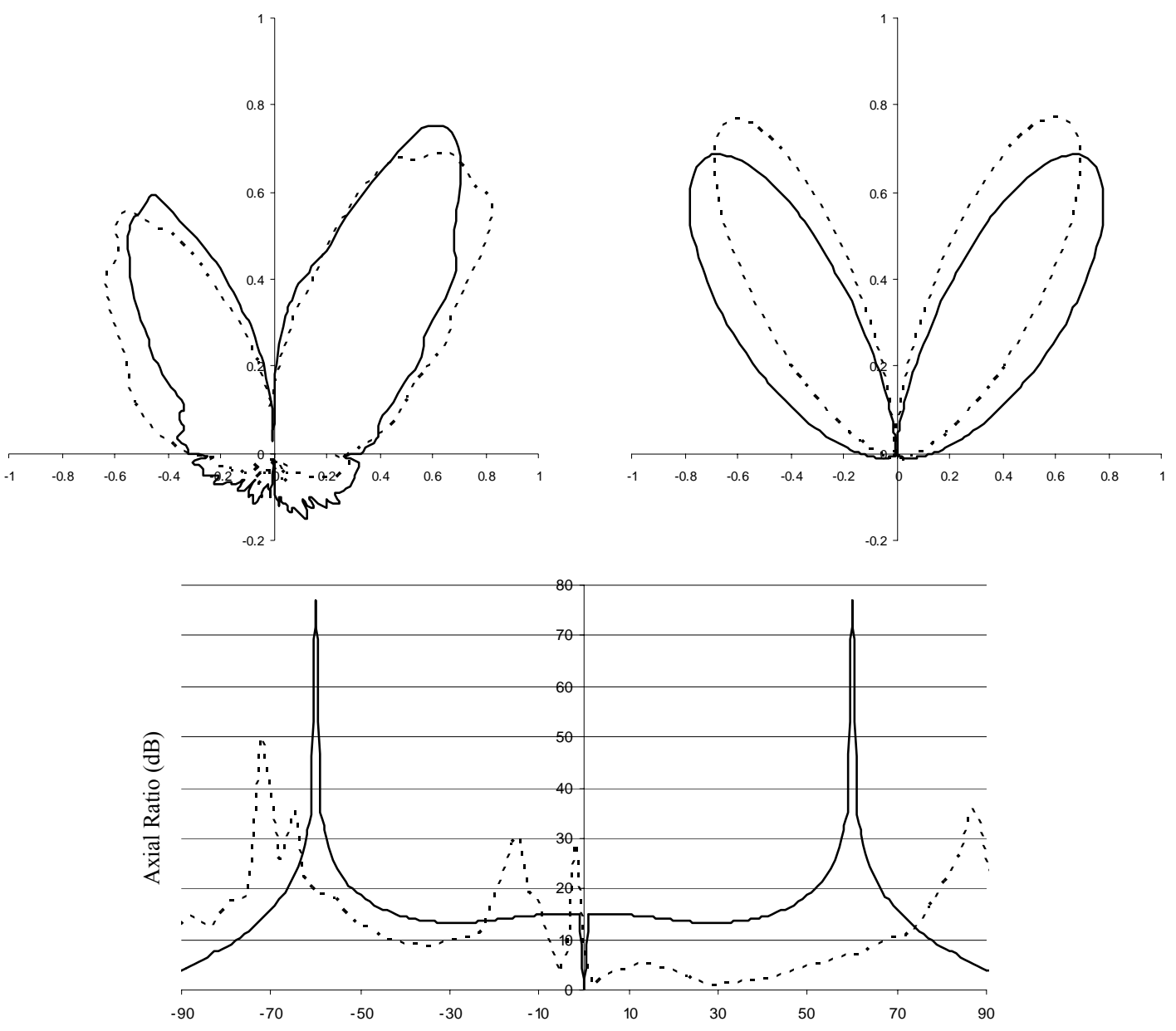

Theta (Degrees)

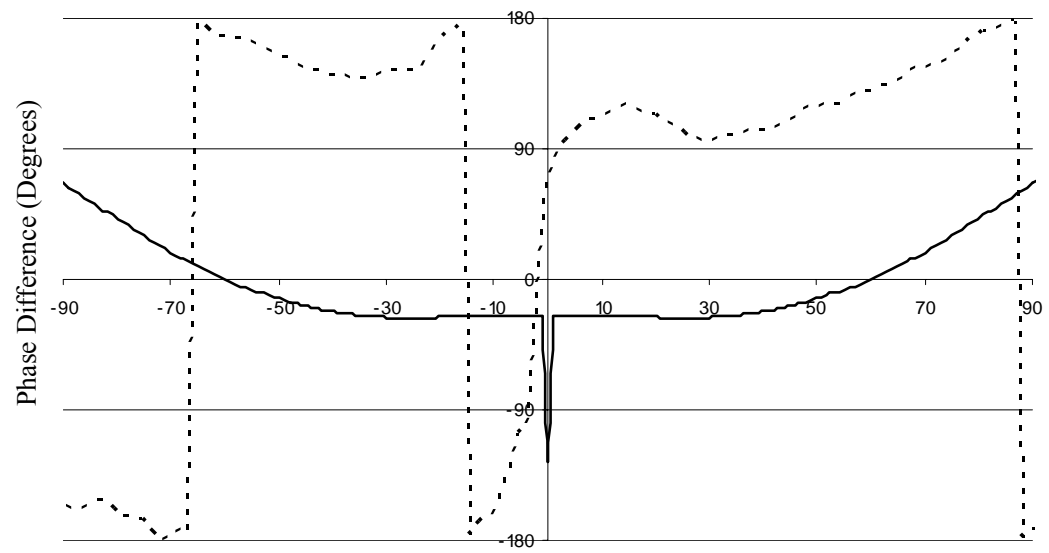

Figure A.7: The measured (top left) and simulated (top right) patterns, with the theta (dashed) and phi (solid) components, and the corresponding axial ratio (middle, in $\mathrm{dB}$ ) and phase difference (bottom, in degrees) plots with the measured (dashed) and simulated (solid) results at a frequency of $3.70 \mathrm{GHz}$ (normalized circumference of $2.98 \lambda$ ). 

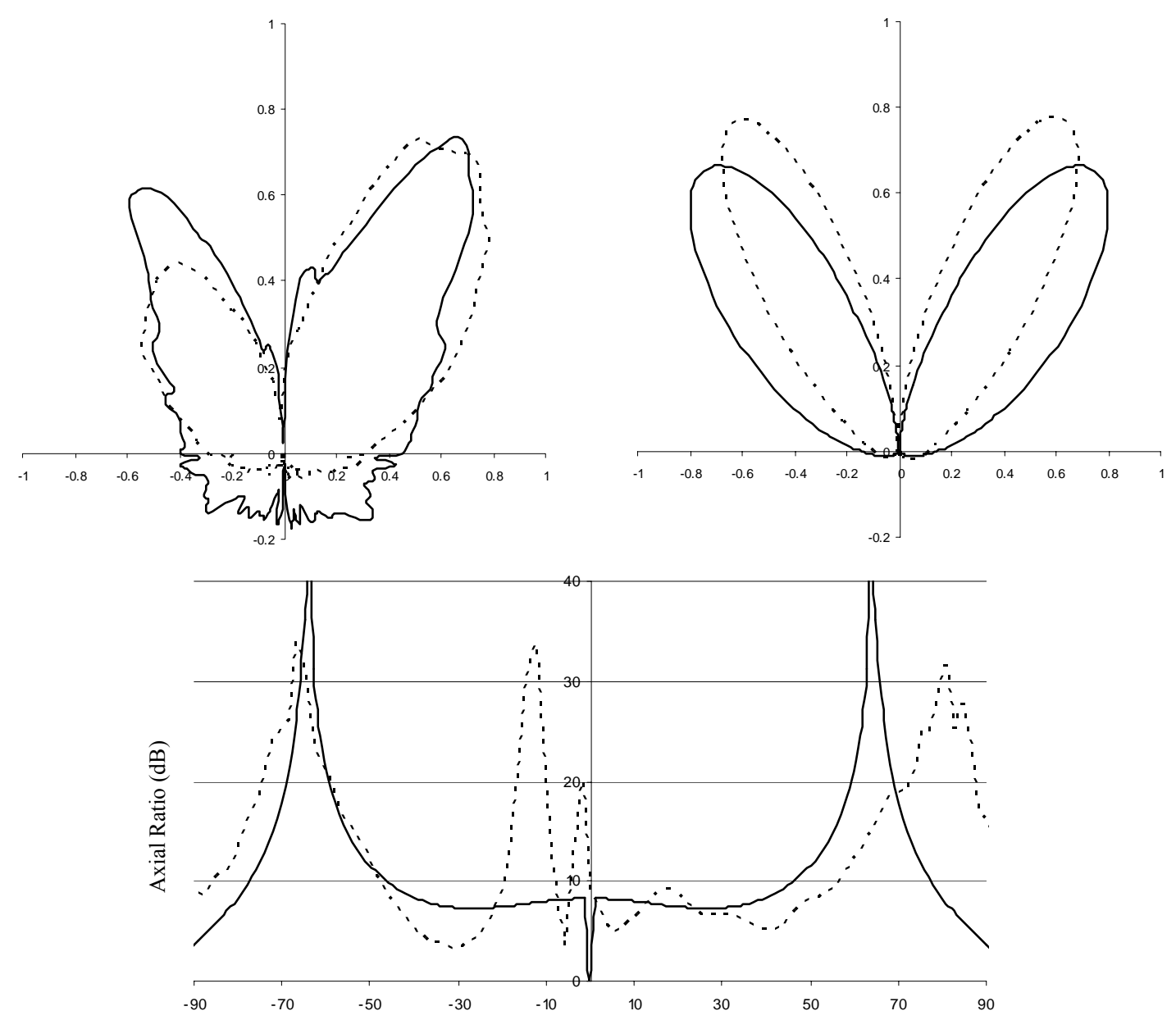

Theta (Degrees)

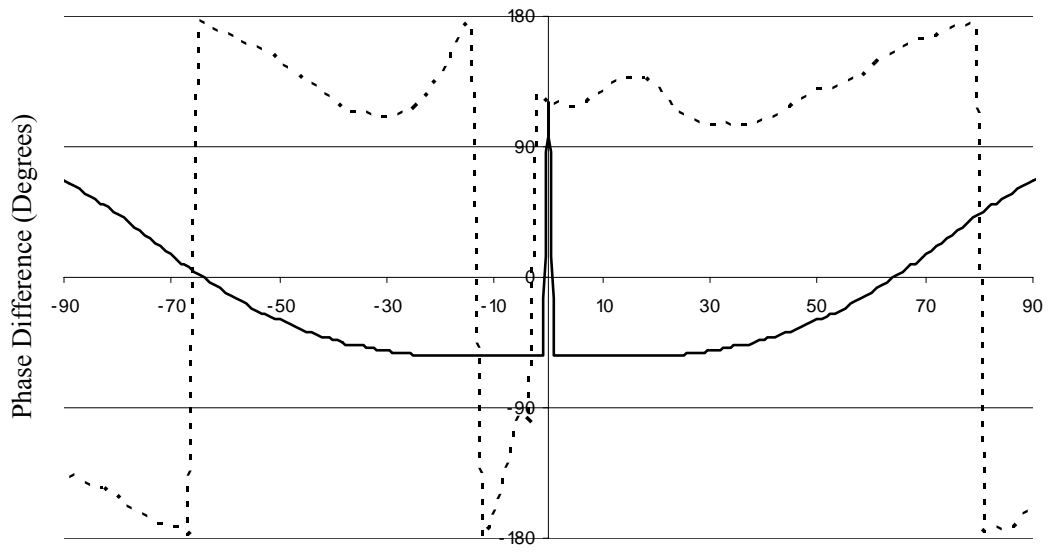

Figure A.8: The measured (top left) and simulated (top right) patterns, with the theta (dashed) and phi (solid) components, and the corresponding axial ratio (middle, in $\mathrm{dB}$ ) and phase difference (bottom, in degrees) plots with the measured (dashed) and simulated (solid) results at a frequency of $3.75 \mathrm{GHz}$ (normalized circumference of $3.02 \lambda$ ). 

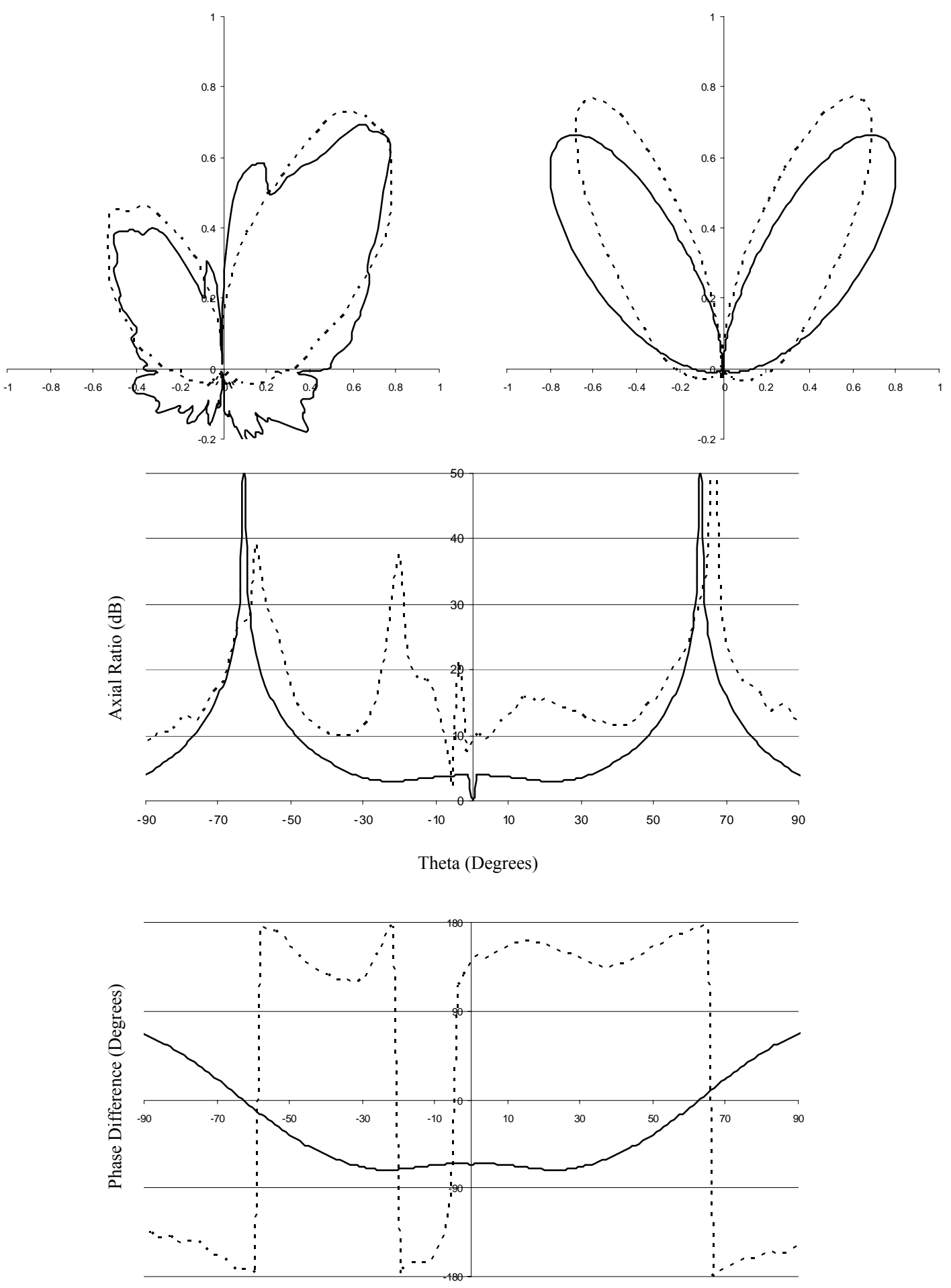

Figure A.9: The measured (top left) and simulated (top right) patterns, with the theta (dashed) and phi (solid) components, and the corresponding axial ratio (middle, in $\mathrm{dB}$ ) and phase difference (bottom, in degrees) plots with the measured (dashed) and simulated (solid) results at a frequency of $3.80 \mathrm{GHz}$ (normalized circumference of $3.06 \lambda$ ). 

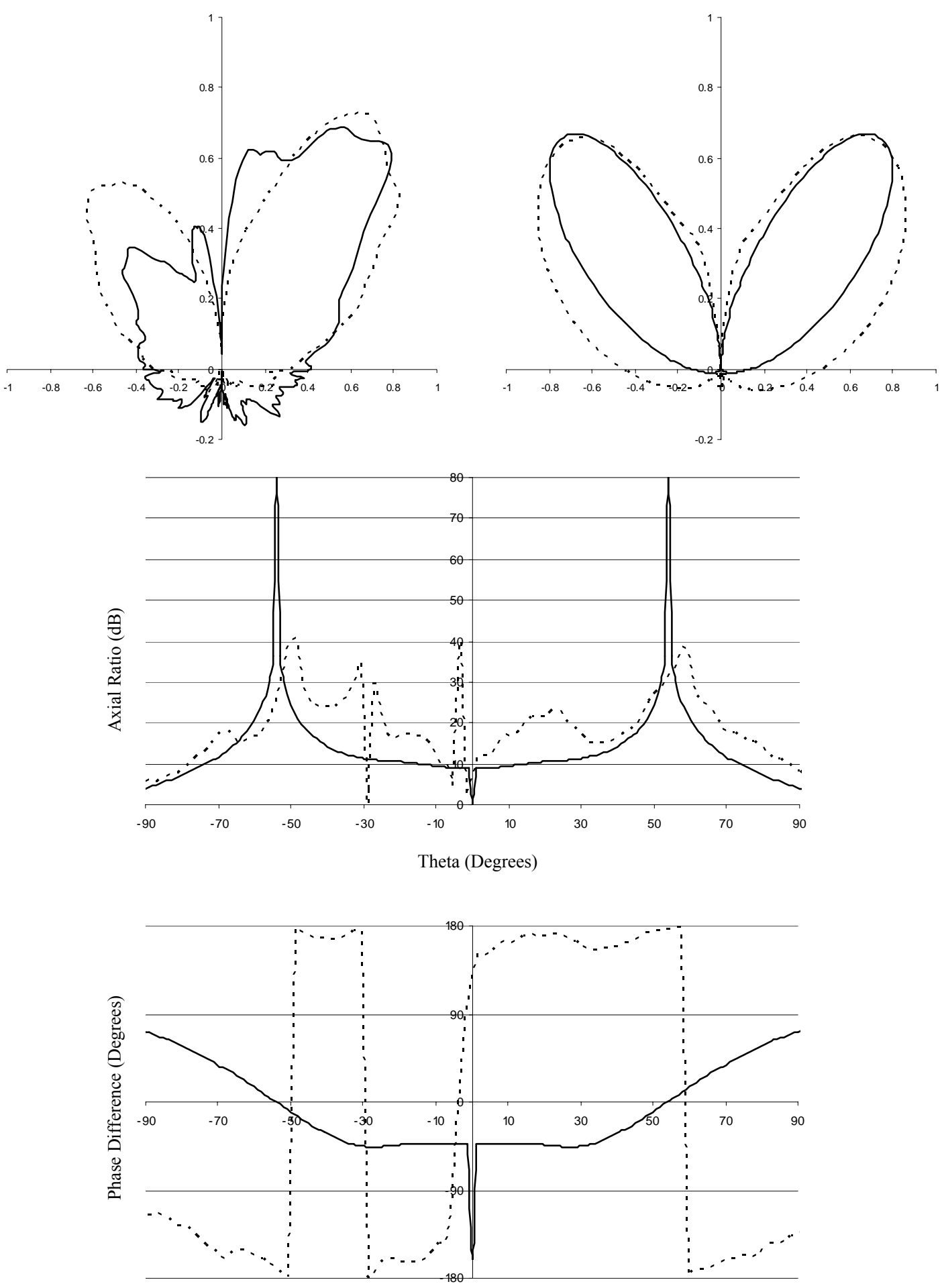

Figure A.10: The measured (top left) and simulated (top right) patterns, with the theta (dashed) and phi (solid) components, and the corresponding axial ratio (middle, in $\mathrm{dB}$ ) and phase difference (bottom, in degrees) plots with the measured (dashed) and simulated (solid) results at a frequency of $3.85 \mathrm{GHz}$ (normalized circumference of $3.10 \lambda$ ). 

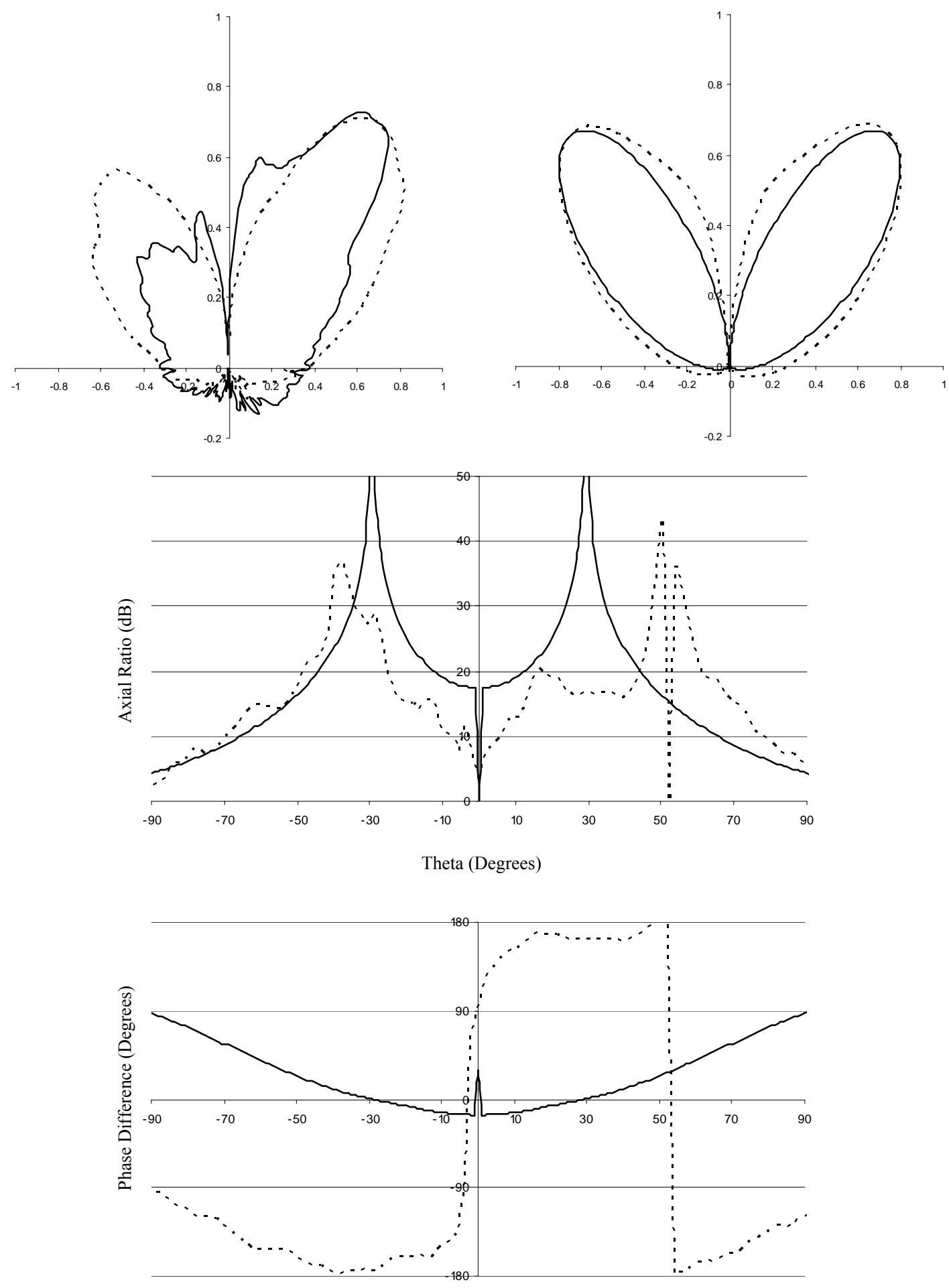

Figure A.11: The measured (top left) and simulated (top right) patterns, with the theta (dashed) and phi (solid) components, and the corresponding axial ratio (middle, in $\mathrm{dB}$ ) and phase difference (bottom, in degrees) plots with the measured (dashed) and simulated (solid) results at a frequency of $3.90 \mathrm{GHz}$ (normalized circumference of $3.14 \lambda$ ). 

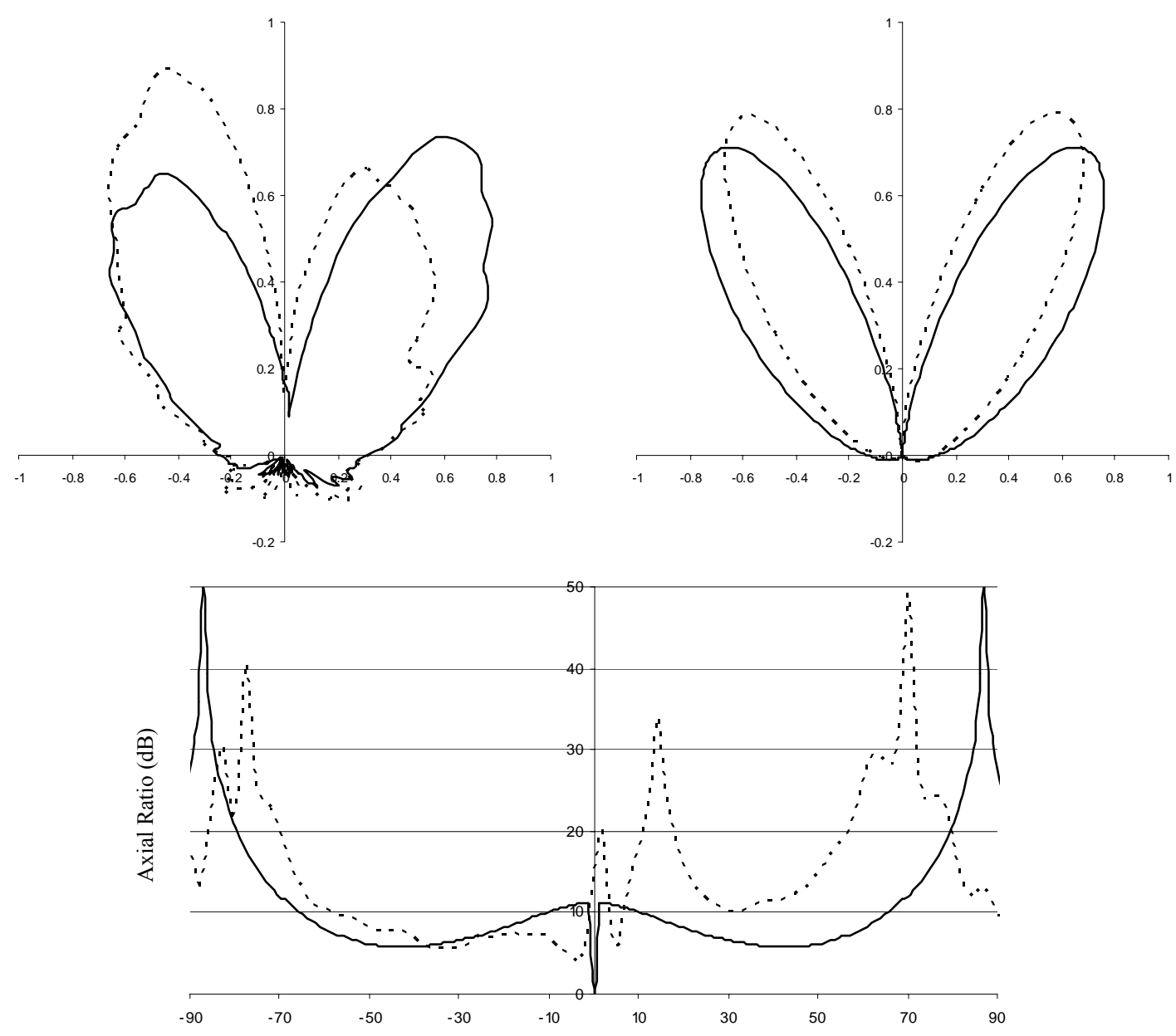

Theta (Degrees)

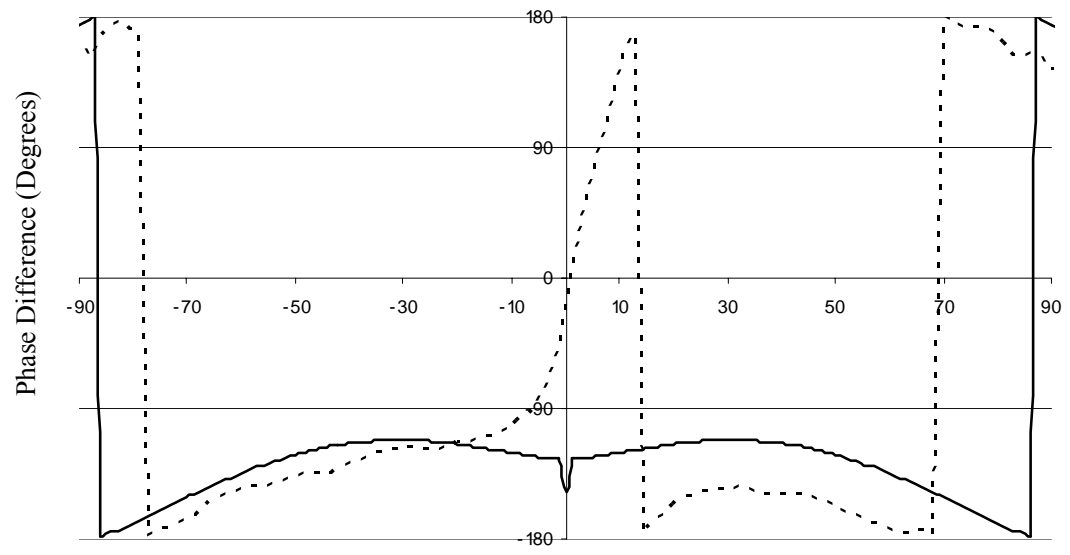

Figure A.12: The measured (top left) and simulated (top right) patterns, with the theta (dashed) and phi (solid) components, and the corresponding axial ratio (middle, in $\mathrm{dB}$ ) and phase difference (bottom, in degrees) plots with the measured (dashed) and simulated (solid) results at a frequency of $3.40 \mathrm{GHz}$ (normalized circumference of $2.74 \lambda$ ). 

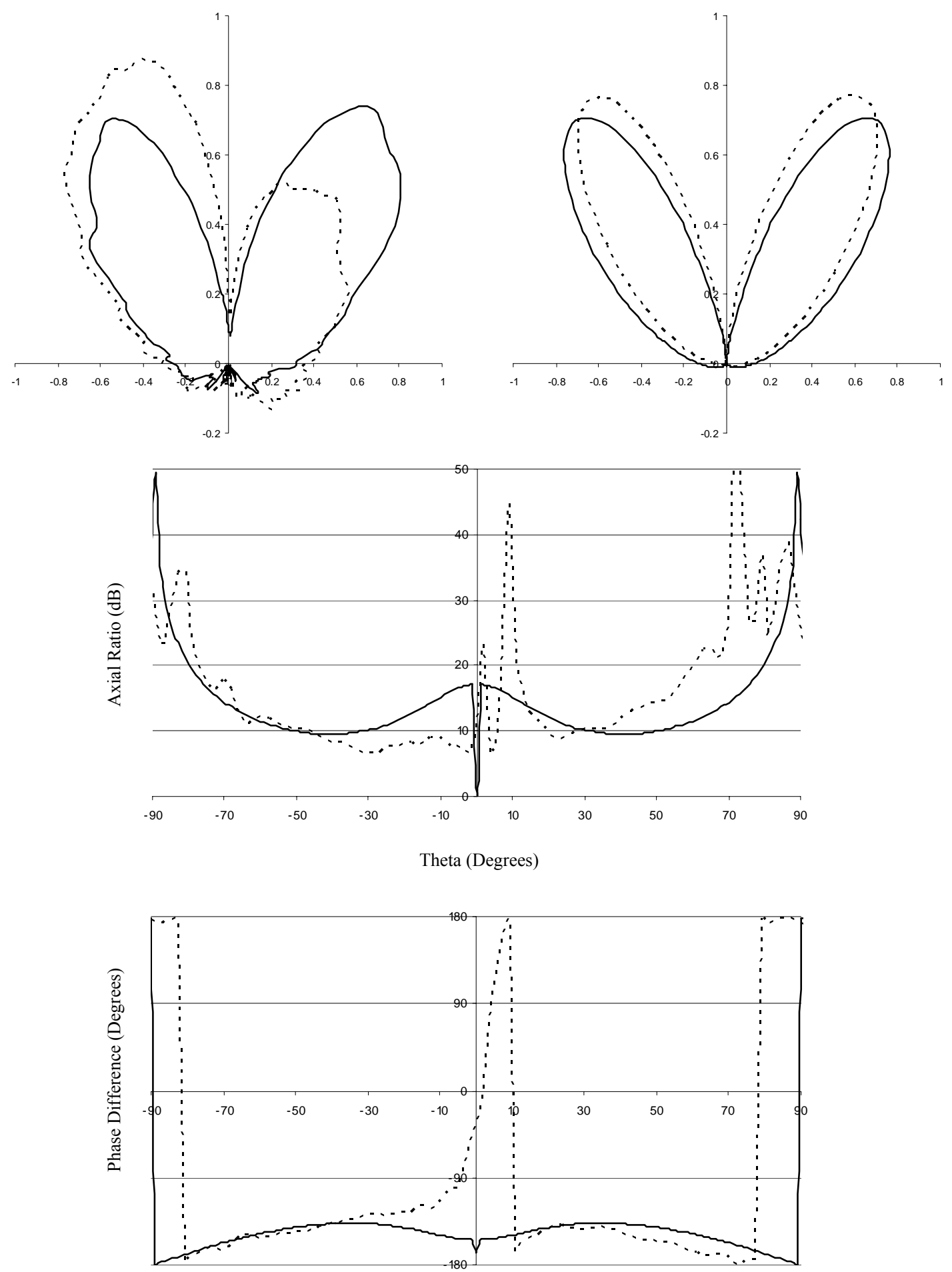

Figure A.13: The measured (top left) and simulated (top right) patterns, with the theta (dashed) and phi (solid) components, and the corresponding axial ratio (middle, in $\mathrm{dB}$ ) and phase difference (bottom, in degrees) plots with the measured (dashed) and simulated (solid) results at a frequency of $3.45 \mathrm{GHz}$ (normalized circumference of $2.78 \lambda$ ). 

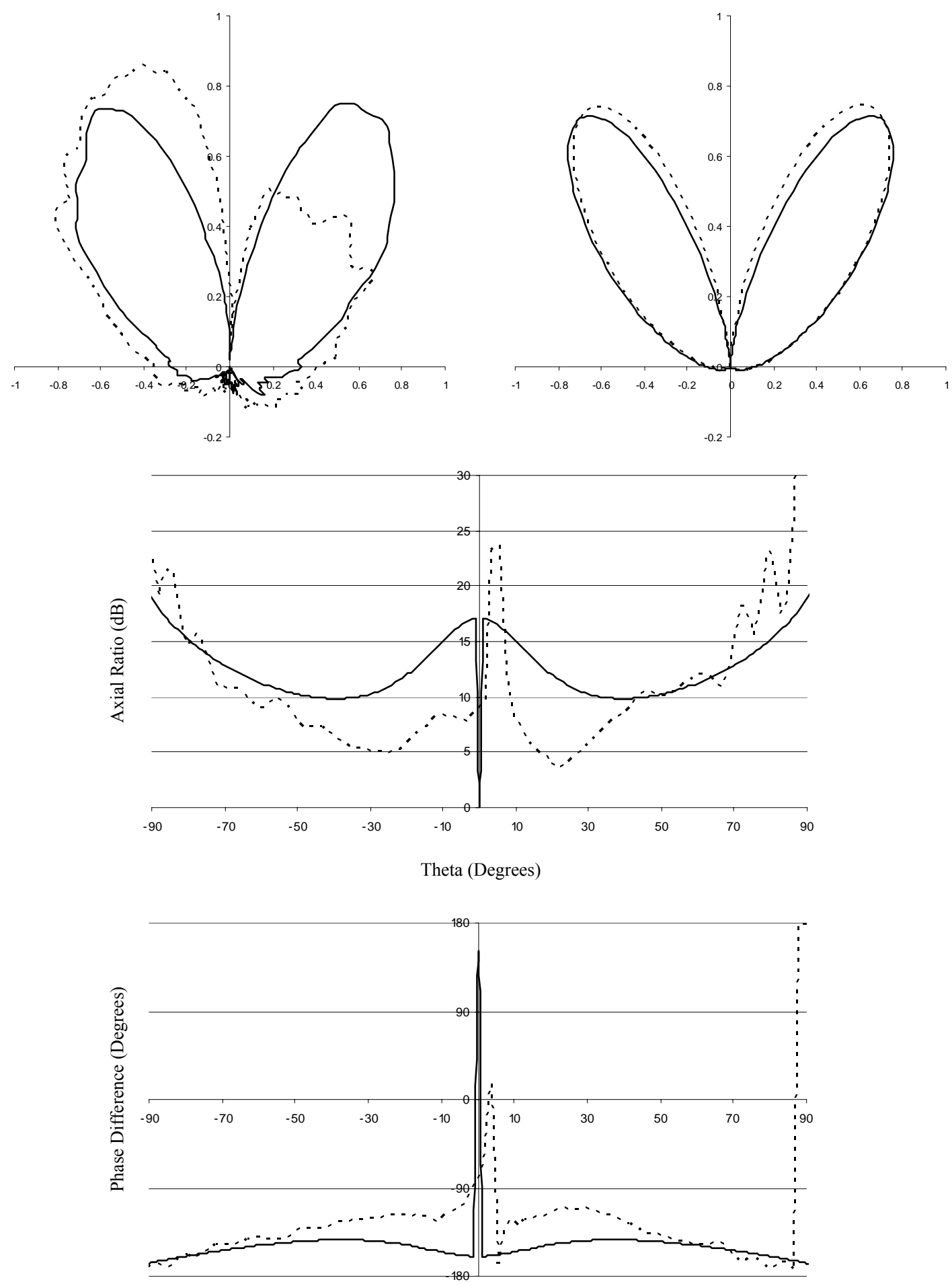

Figure A.14: The measured (top left) and simulated (top right) patterns, with the theta (dashed) and phi (solid) components, and the corresponding axial ratio (middle, in $\mathrm{dB}$ ) and phase difference (bottom, in degrees) plots with the measured (dashed) and simulated (solid) results at a frequency of $3.50 \mathrm{GHz}$ (normalized circumference of $2.82 \lambda$ ). 

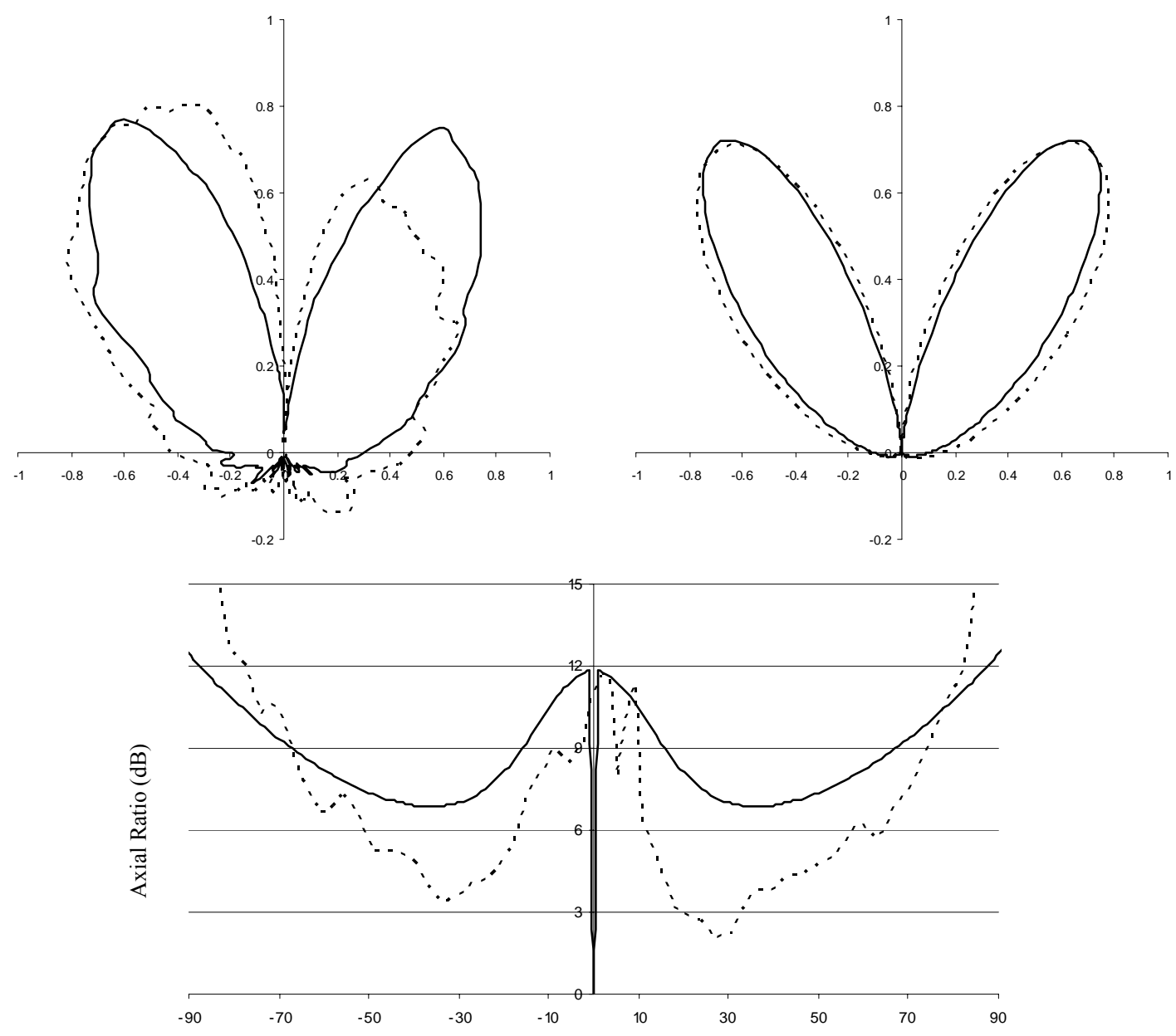

Theta (Degrees)

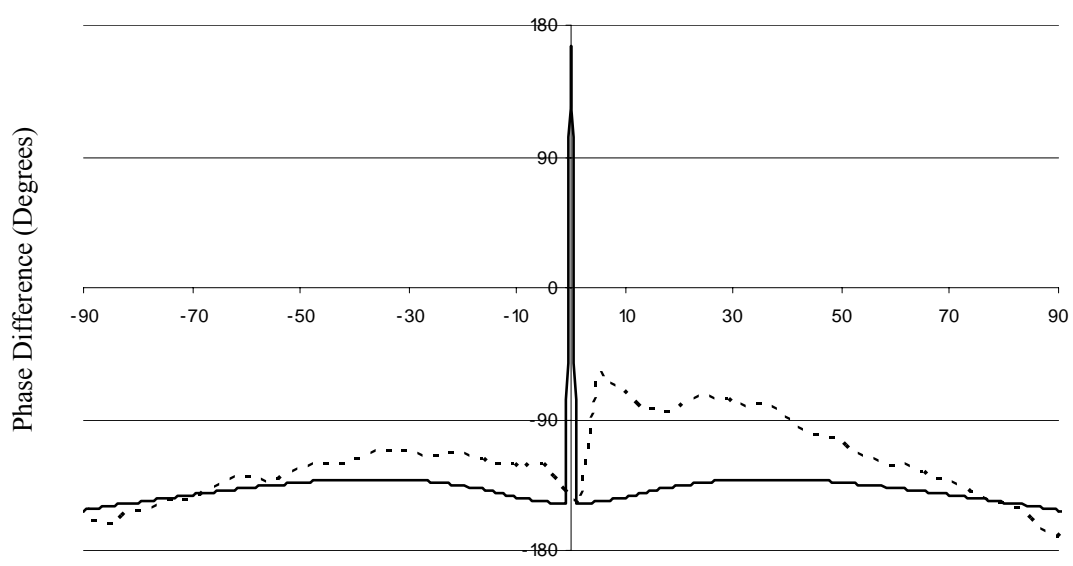

Figure A.15: The measured (top left) and simulated (top right) patterns, with the theta (dashed) and phi (solid) components, and the corresponding axial ratio (middle, in $\mathrm{dB}$ ) and phase difference (bottom, in degrees) plots with the measured (dashed) and simulated (solid) results at a frequency of $3.55 \mathrm{GHz}$ (normalized circumference of $2.86 \lambda$ ). 

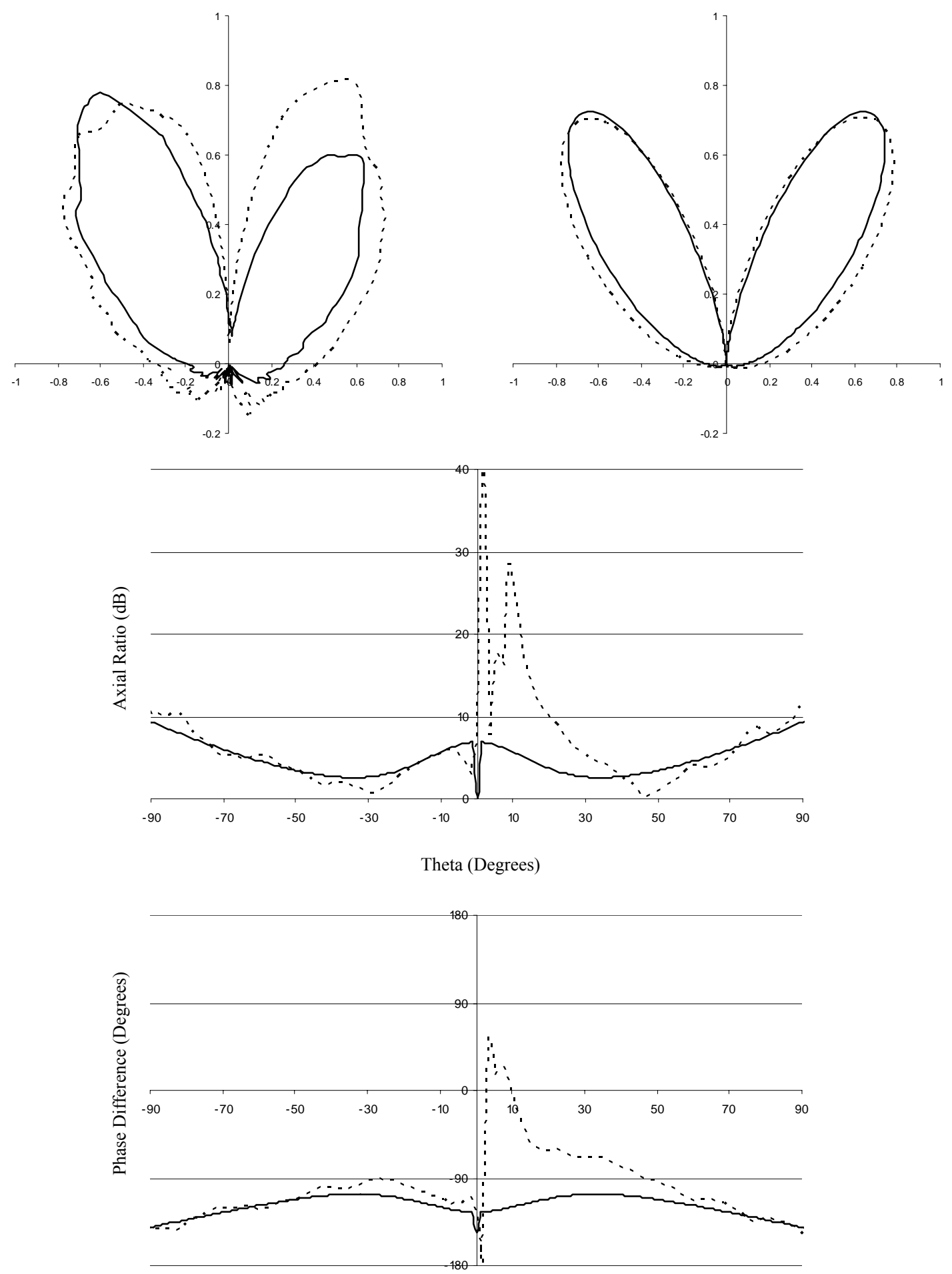

Figure A.16: The measured (top left) and simulated (top right) patterns, with the theta (dashed) and phi (solid) components, and the corresponding axial ratio (middle, in $\mathrm{dB}$ ) and phase difference (bottom, in degrees) plots with the measured (dashed) and simulated (solid) results at a frequency of $3.60 \mathrm{GHz}$ (normalized circumference of $2.90 \lambda$ ). 

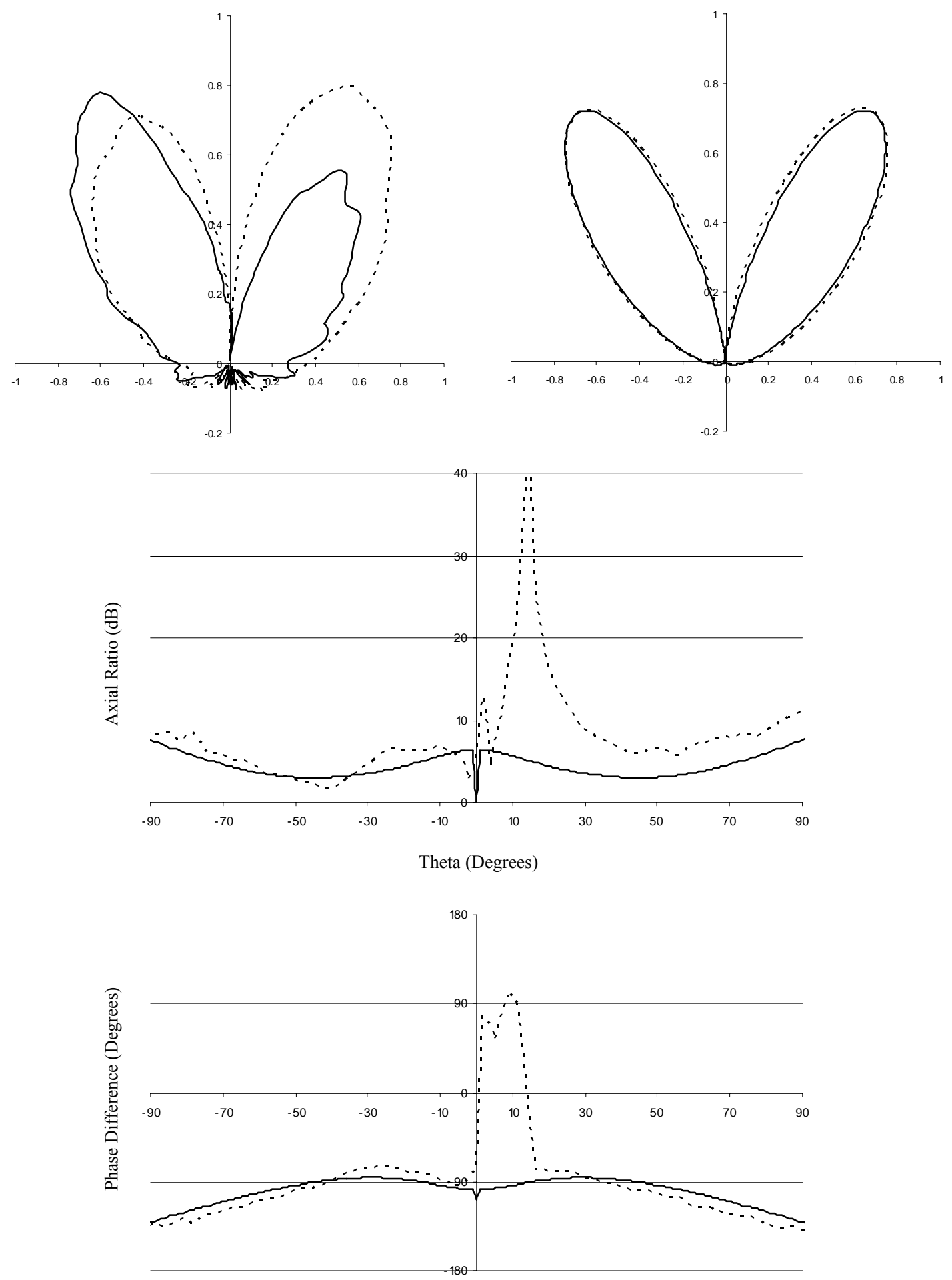

Figure A.17: The measured (top left) and simulated (top right) patterns, with the theta (dashed) and phi (solid) components, and the corresponding axial ratio (middle, in $\mathrm{dB}$ ) and phase difference (bottom, in degrees) plots with the measured (dashed) and simulated (solid) results at a frequency of $3.65 \mathrm{GHz}$ (normalized circumference of $2.94 \lambda$ ). 

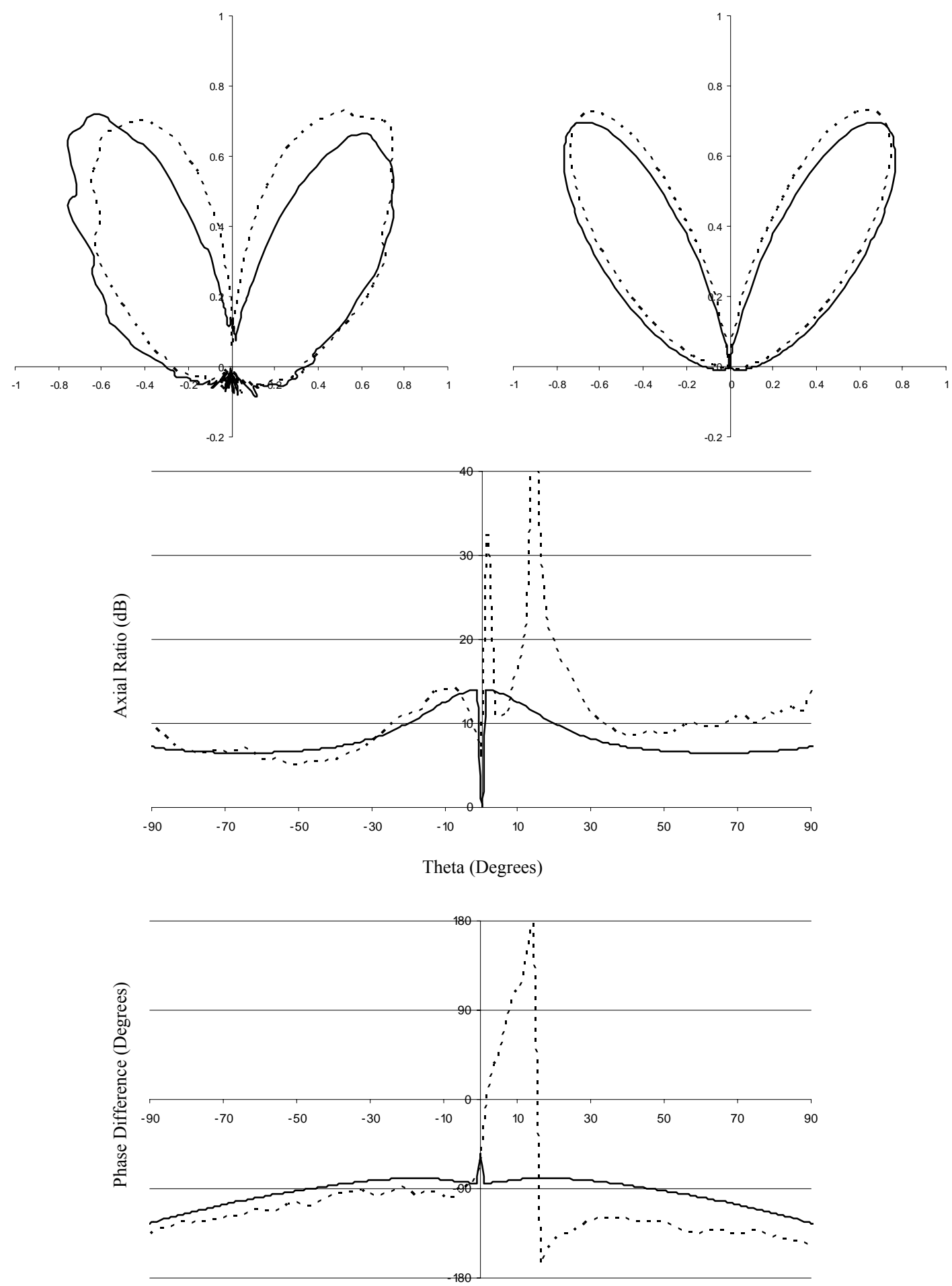

Figure A.18: The measured (top left) and simulated (top right) patterns, with the theta (dashed) and phi (solid) components, and the corresponding axial ratio (middle, in $\mathrm{dB}$ ) and phase difference (bottom, in degrees) plots with the measured (dashed) and simulated (solid) results at a frequency of $3.70 \mathrm{GHz}$ (normalized circumference of $2.98 \lambda$ ). 

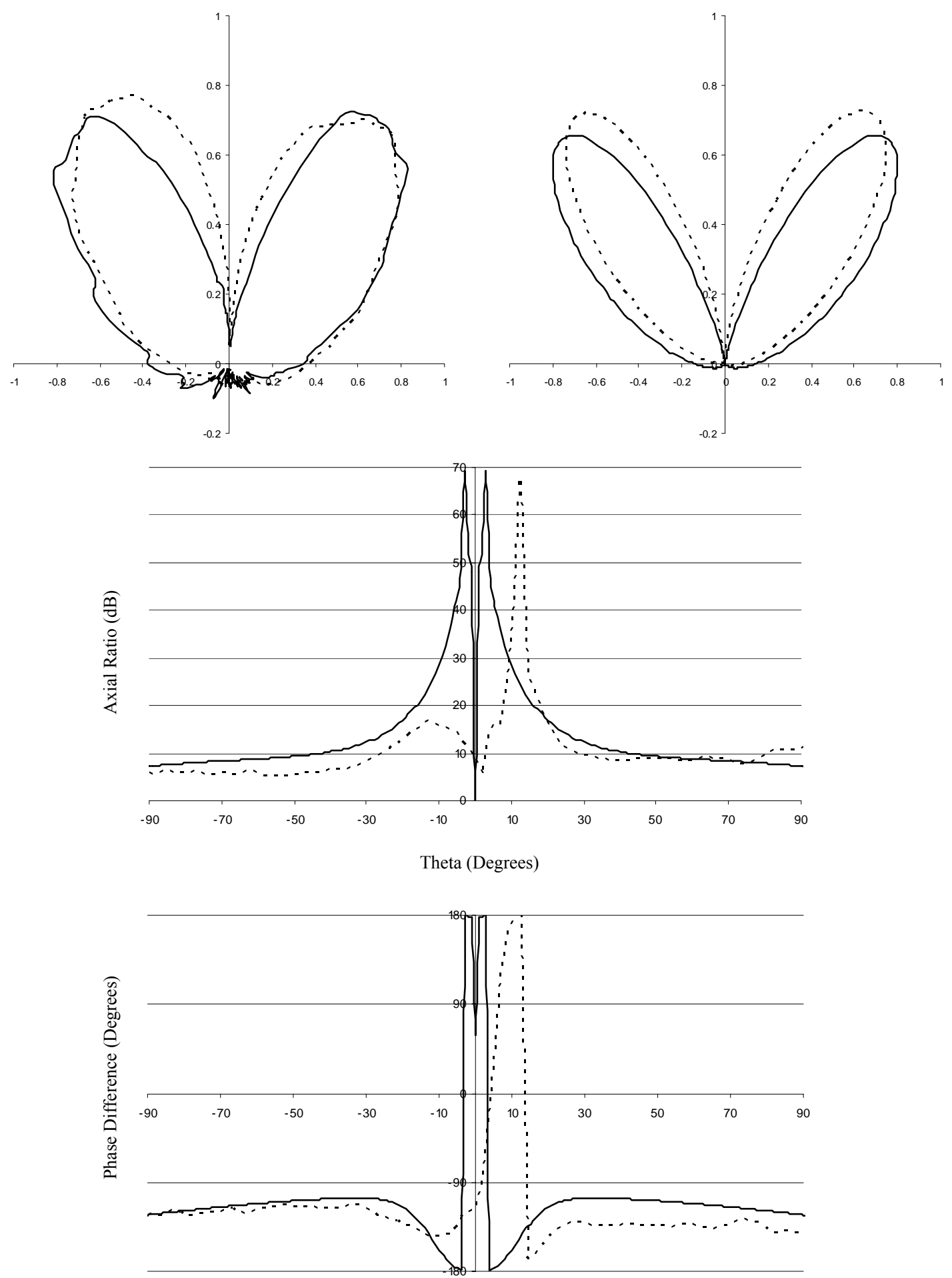

Figure A.19: The measured (top left) and simulated (top right) patterns, with the theta (dashed) and phi (solid) components, and the corresponding axial ratio (middle, in $\mathrm{dB}$ ) and phase difference (bottom, in degrees) plots with the measured (dashed) and simulated (solid) results at a frequency of $3.75 \mathrm{GHz}$ (normalized circumference of $3.02 \lambda$ ). 

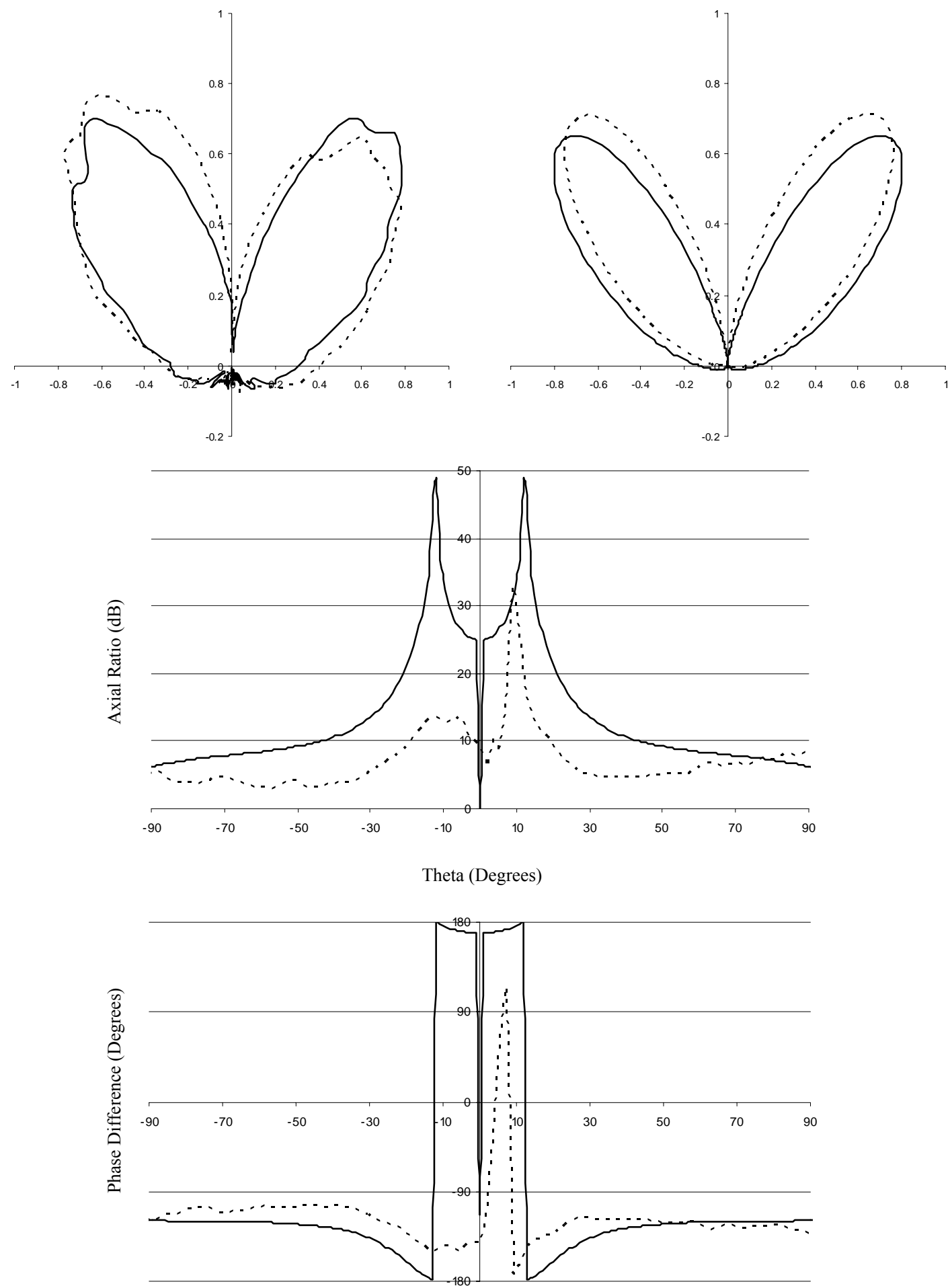

Figure A.20: The measured (top left) and simulated (top right) patterns, with the theta (dashed) and phi (solid) components, and the corresponding axial ratio (middle, in $\mathrm{dB}$ ) and phase difference (bottom, in degrees) plots with the measured (dashed) and simulated (solid) results at a frequency of $3.80 \mathrm{GHz}$ (normalized circumference of $3.06 \lambda$ ). 

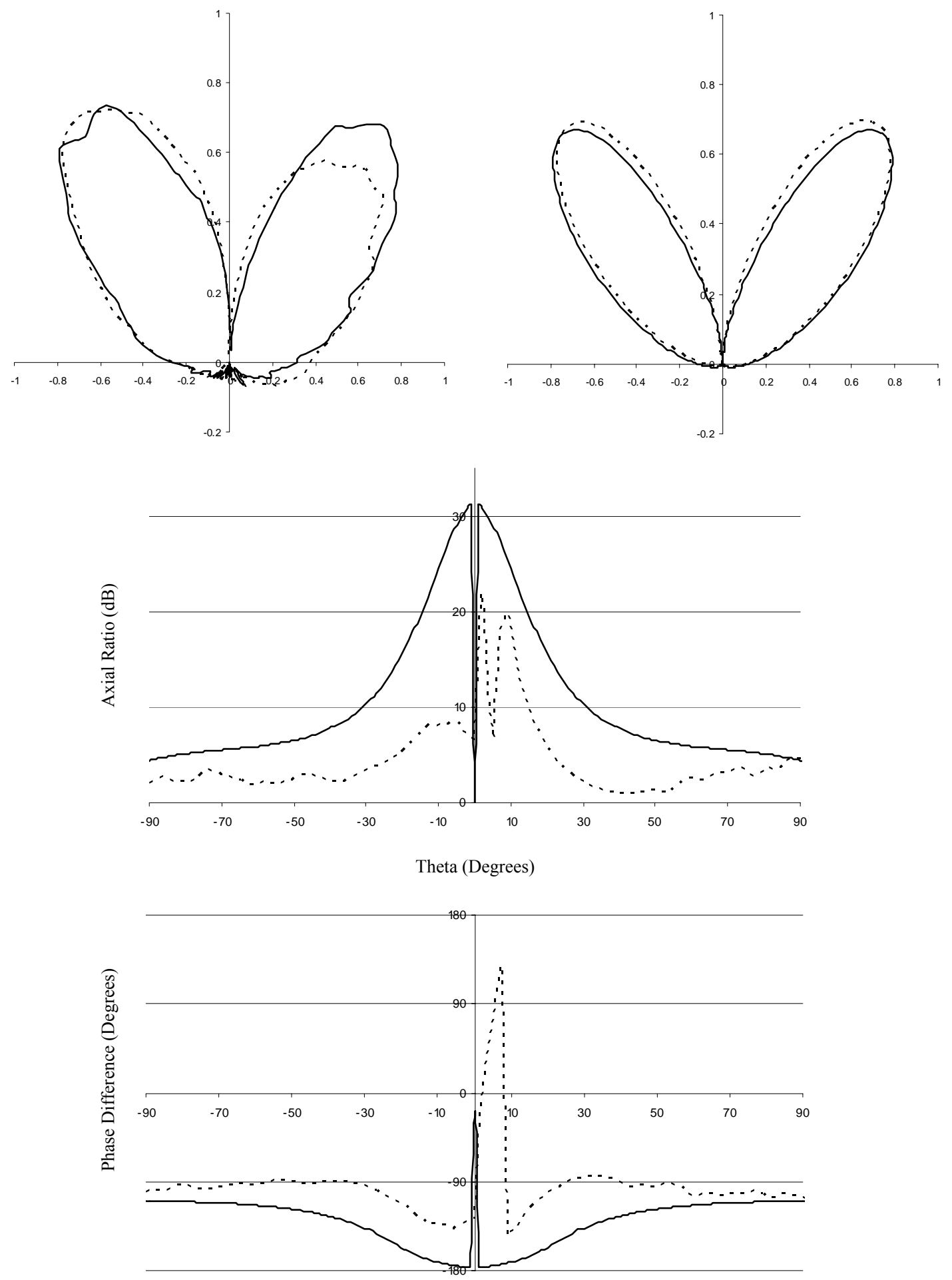

Figure A.21: The measured (top left) and simulated (top right) patterns, with the theta (dashed) and phi (solid) components, and the corresponding axial ratio (middle, in $\mathrm{dB}$ ) and phase difference (bottom, in degrees) plots with the measured (dashed) and simulated (solid) results at a frequency of $3.85 \mathrm{GHz}$ (normalized circumference of $3.10 \lambda$ ). 

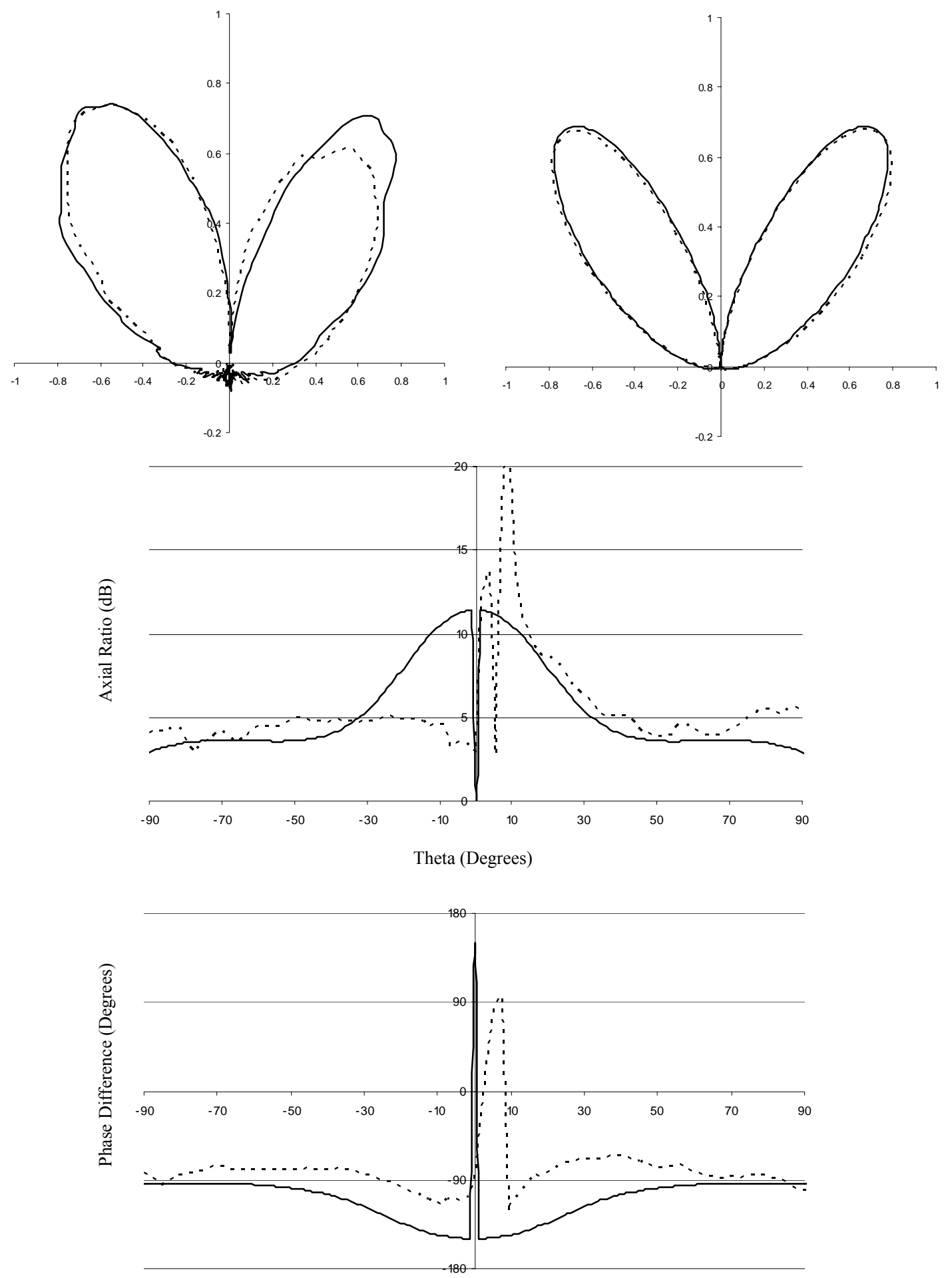

Figure A.22: The measured (top left) and simulated (top right) patterns, with the theta (dashed) and phi (solid) components, and the corresponding axial ratio (middle, in $\mathrm{dB}$ ) and phase difference (bottom, in degrees) plots with the measured (dashed) and simulated (solid) results at a frequency of $3.90 \mathrm{GHz}$ (normalized circumference of $3.14 \lambda$ ). 


\section{Appendix B: Quadrifilar Hemispherical Helix Simulated Radiation Pattern}

Both the simulated and measured radiation patterns for the quadrifilar hemispherical helical antenna are herein shown in their entirety. Figures B.1 through B.3 are E-plane data, and Figures B.12 through B.22 are H-plane data. All pattern plots are shown using a linear scale to facilitate a more visually descriptive rendering of the results which may sometimes be impeded by the use of logarithmic plots. Also included for each pattern is the axial ratio (in $\mathrm{dB}$ ) and phase difference (in degrees) over the region of space above the ground plane. 

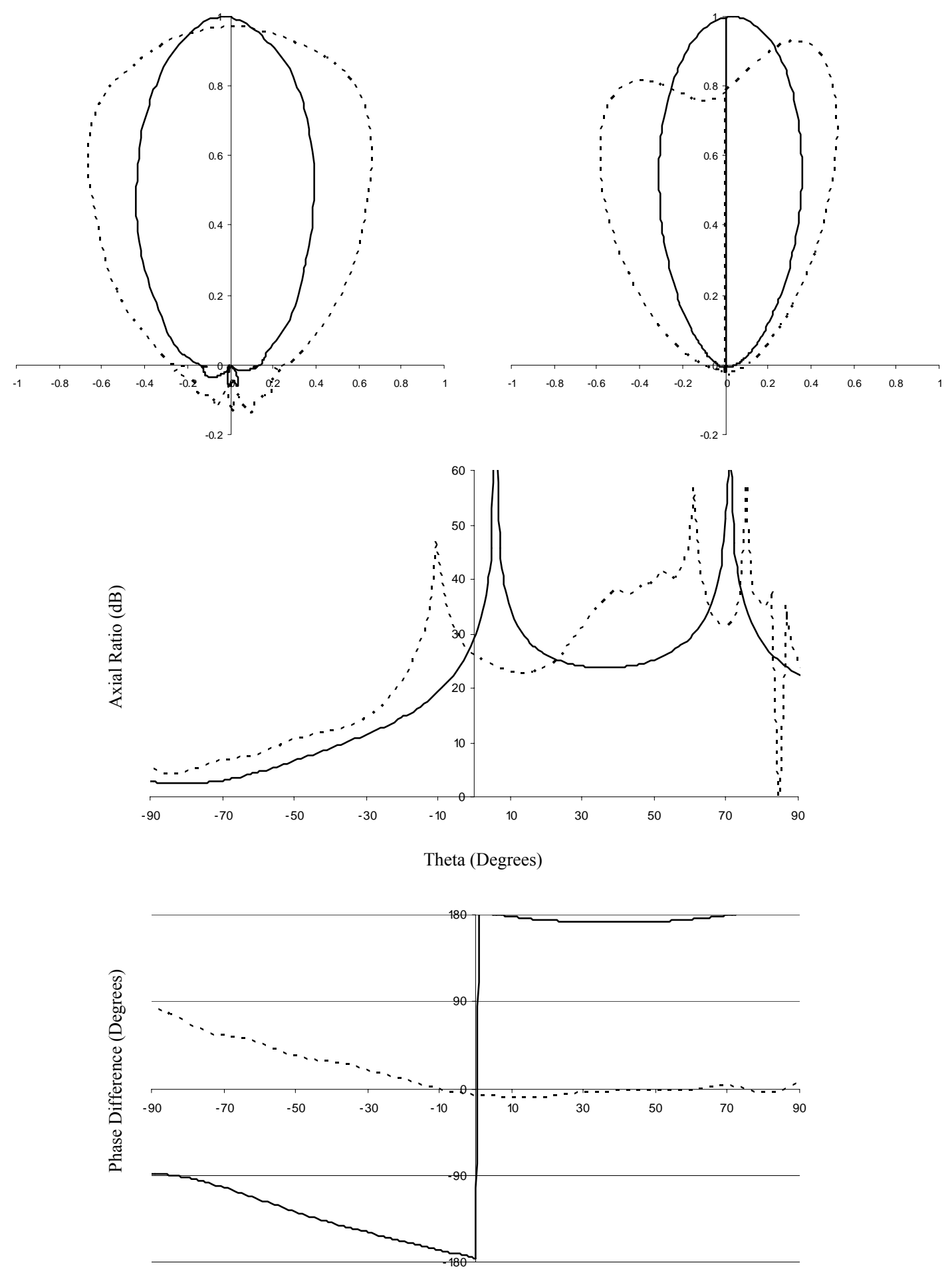

Figure B.1: The measured (top left) and simulated (top right) patterns, with the theta (dashed) and phi (solid) components, and the corresponding axial ratio (middle, in $\mathrm{dB}$ ) and phase difference (bottom, in degrees) plots with the measured (dashed) and simulated (solid) results at a frequency of $1.15 \mathrm{GHz}$ (normalized circumference of $0.73 \lambda$ ). 

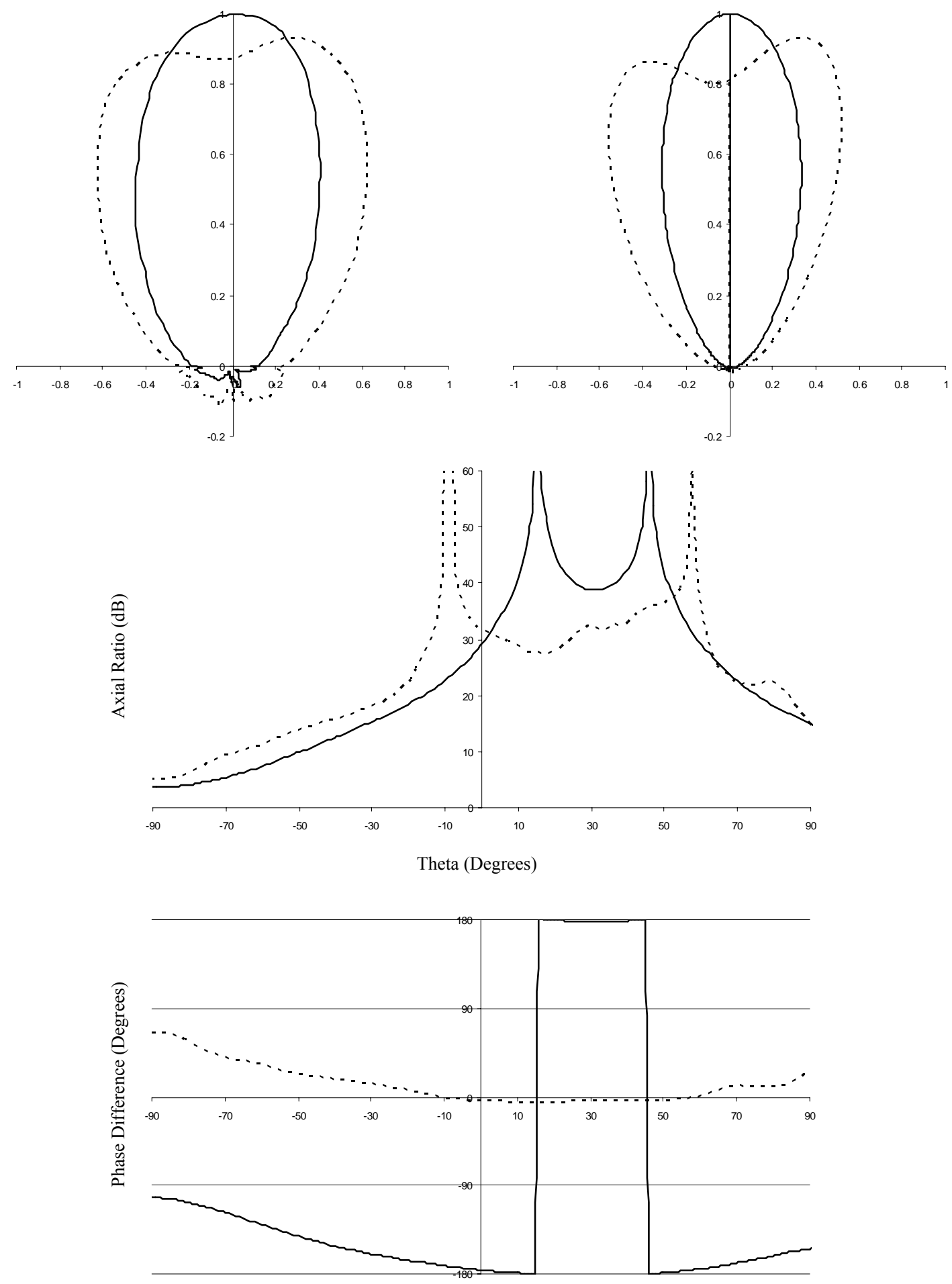

Figure B.2: The measured (top left) and simulated (top right) patterns, with the theta (dashed) and phi (solid) components, and the corresponding axial ratio (middle, in $\mathrm{dB}$ ) and phase difference (bottom, in degrees) plots with the measured (dashed) and simulated (solid) results at a frequency of $1.20 \mathrm{GHz}$ (normalized circumference of $0.77 \lambda$ ). 

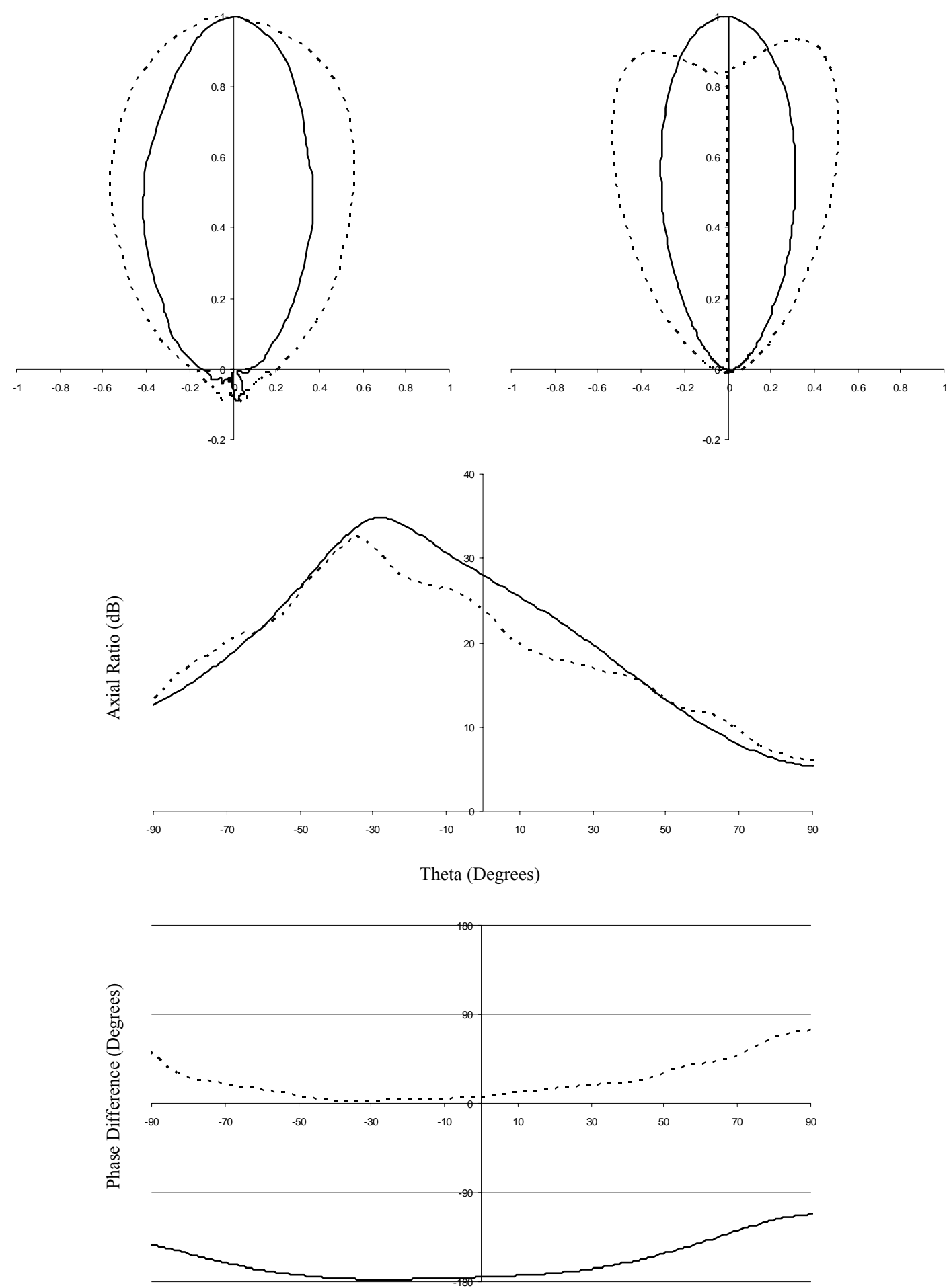

Figure B.3: The measured (top left) and simulated (top right) patterns, with the theta (dashed) and phi (solid) components, and the corresponding axial ratio (middle, in $\mathrm{dB}$ ) and phase difference (bottom, in degrees) plots with the measured (dashed) and simulated (solid) results at a frequency of $1.25 \mathrm{GHz}$ (normalized circumference of $0.80 \lambda$ ). 

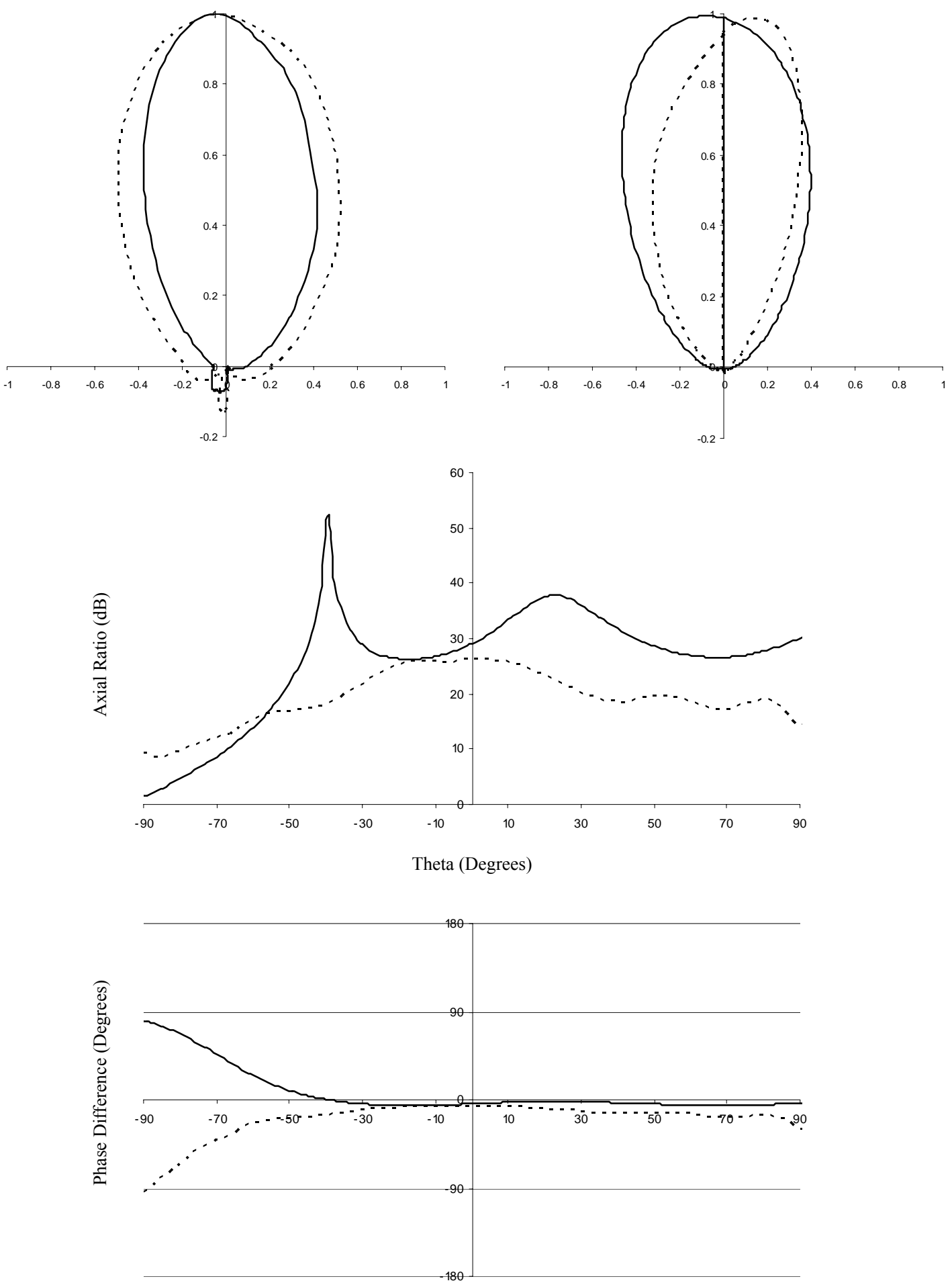

Figure B.4: The measured (top left) and simulated (top right) patterns, with the theta (dashed) and phi (solid) components, and the corresponding axial ratio (middle, in $\mathrm{dB}$ ) and phase difference (bottom, in degrees) plots with the measured (dashed) and simulated (solid) results at a frequency of $1.15 \mathrm{GHz}$ (normalized circumference of $0.73 \lambda$ ). 

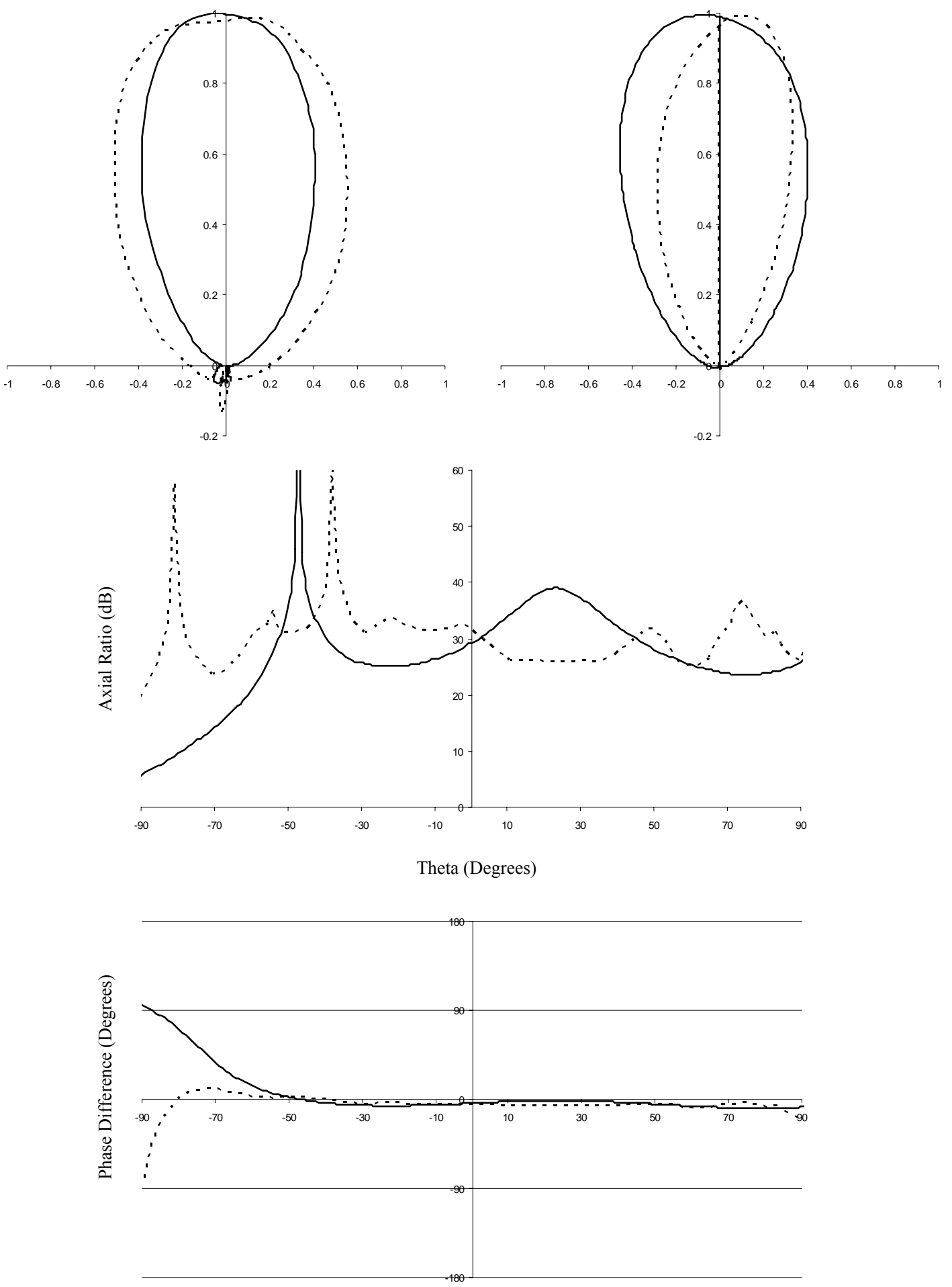

Figure B.5: The measured (top left) and simulated (top right) patterns, with the theta (dashed) and phi (solid) components, and the corresponding axial ratio (middle, in $\mathrm{dB}$ ) and phase difference (bottom, in degrees) plots with the measured (dashed) and simulated (solid) results at a frequency of $1.20 \mathrm{GHz}$ (normalized circumference of $0.77 \lambda$ ). 

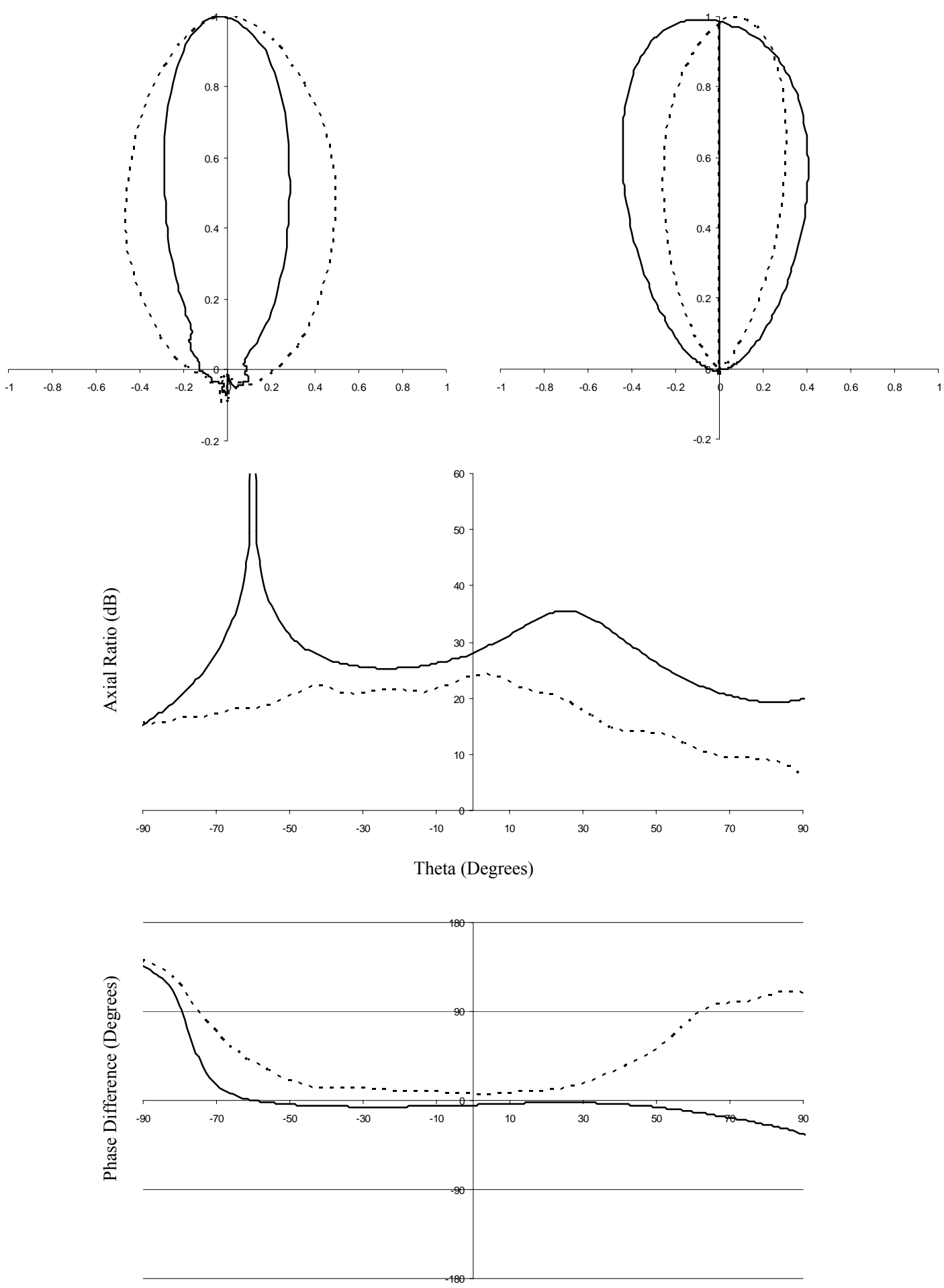

Figure B.6: The measured (top left) and simulated (top right) patterns, with the theta (dashed) and phi (solid) components, and the corresponding axial ratio (middle, in $\mathrm{dB}$ ) and phase difference (bottom, in degrees) plots with the measured (dashed) and simulated (solid) results at a frequency of $1.25 \mathrm{GHz}$ (normalized circumference of $0.80 \lambda$ ). 


\section{Appendix C. Input Impedance Plots}

Since the voltage standing wave ratio may not be an entirely accurate

characterization of the antenna, the input impedance has been plotted for the two antenna

designs. For both the quadrifilar and bifilar hemispherical helices, a plot of the measured and simulated real (resistance) and imaginary (reactance) parts of the input impedance are shown.

As can be seen from the results in Figures C.1 through C.4, despite the fact that the magnitudes of the measured and simulated values differ significantly, the general trends are the same. 


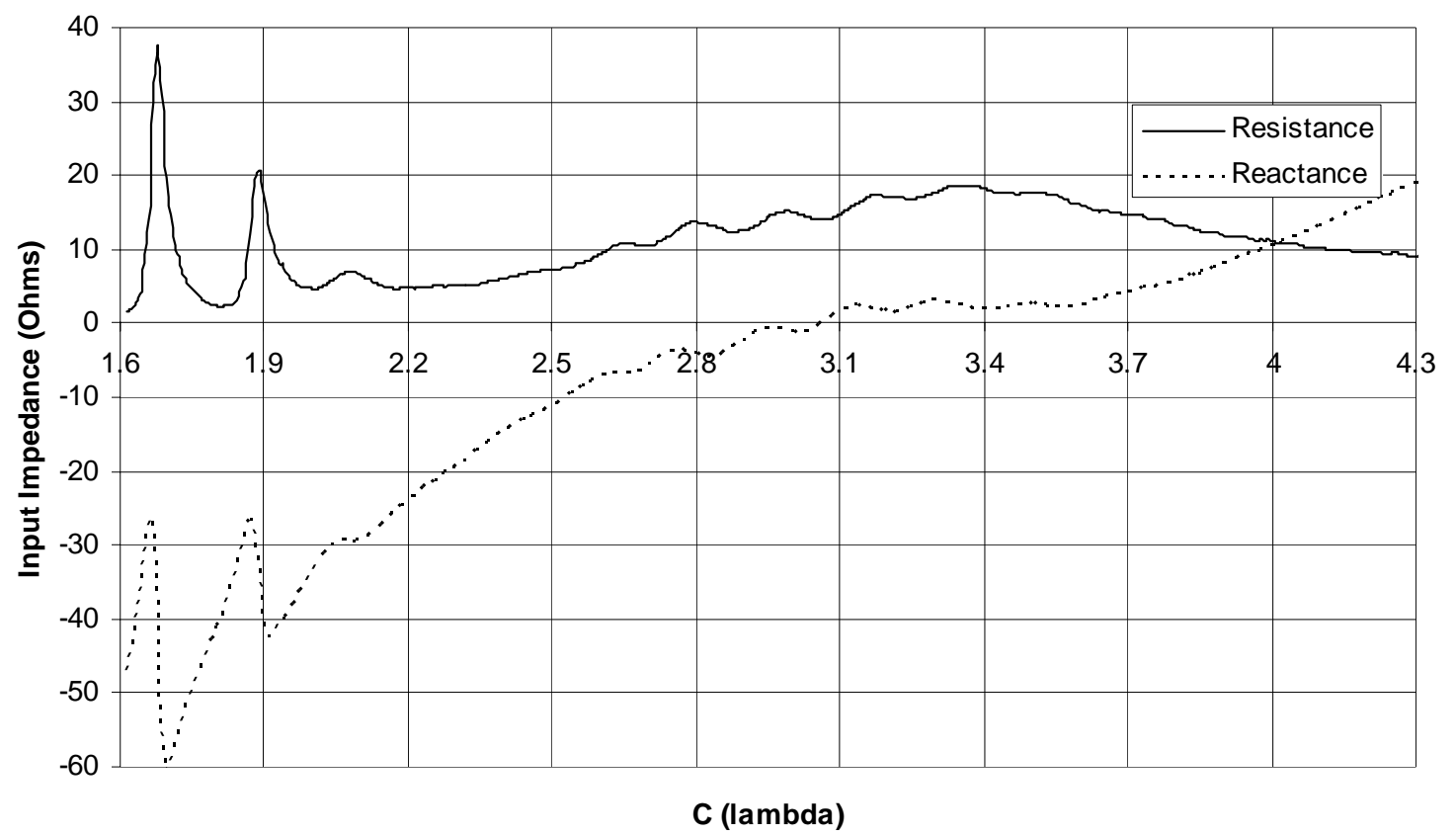

Figure C.1: Measured input impedance data for the bifilar hemispherical helix, with the resistance (solid) and reactance (dashed) components shown.

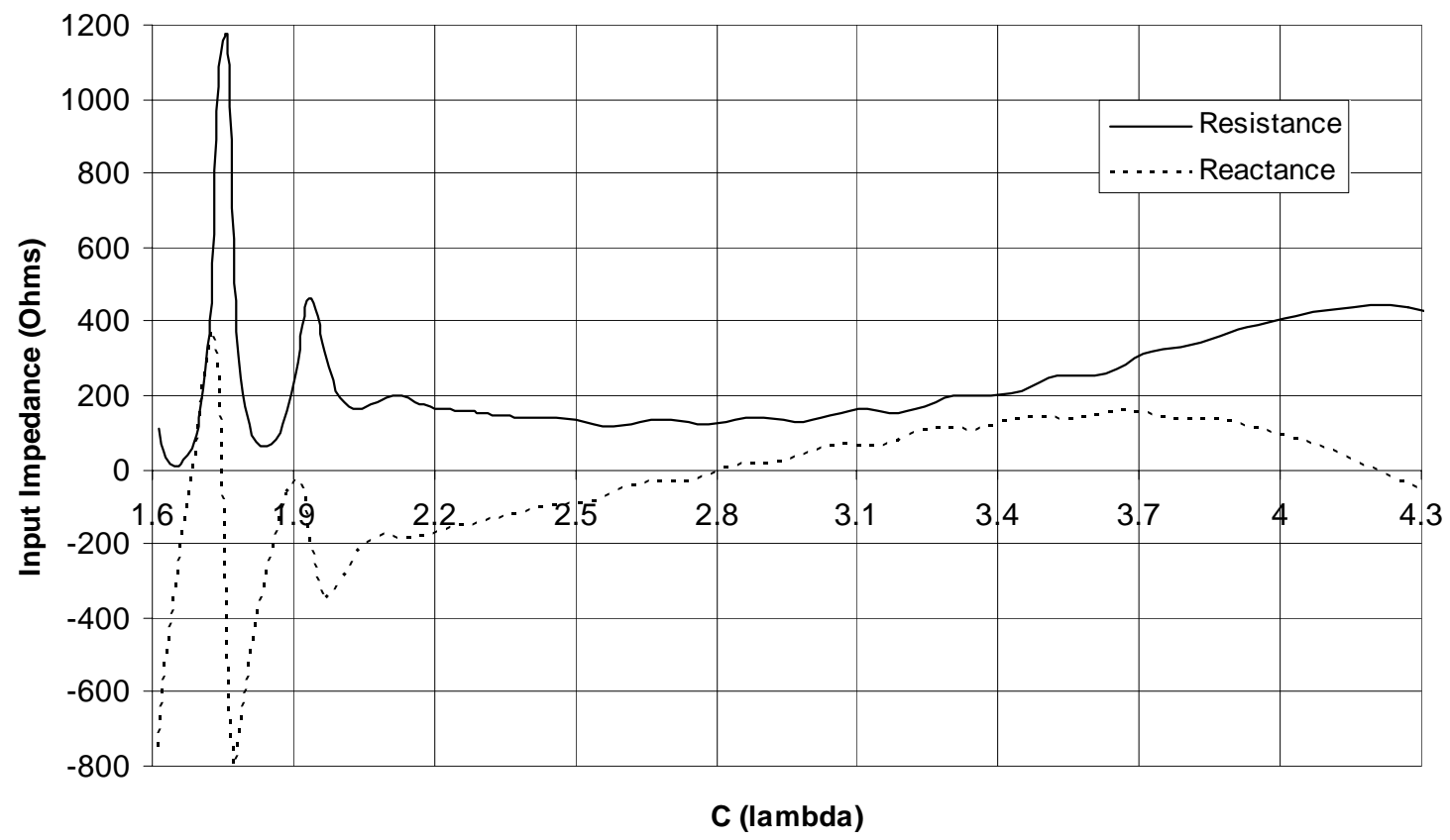

Figure C.2: Simulated input impedance data for the bifilar hemispherical helix, with the resistance (solid) and reactance (dashed) components shown. 


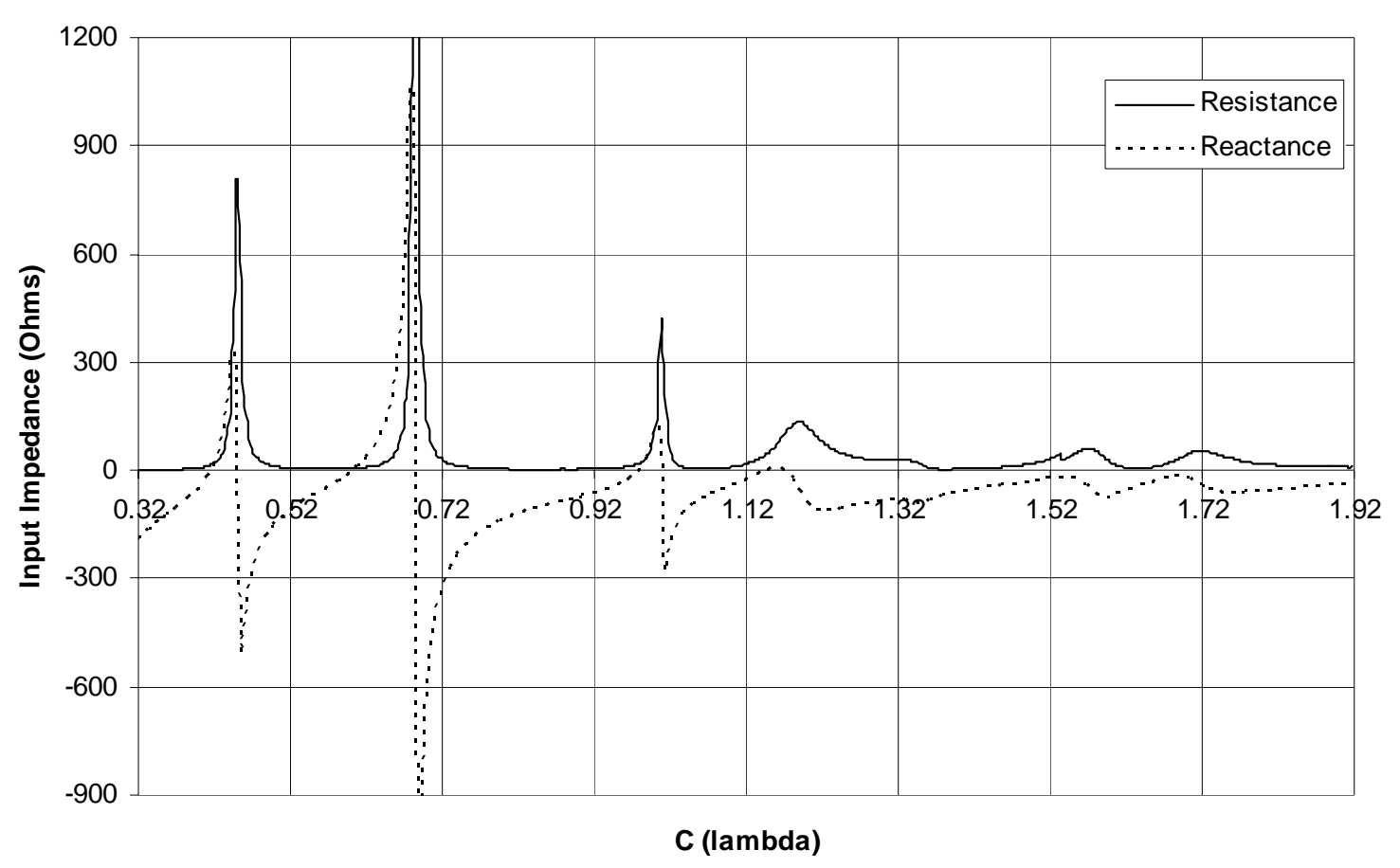

Figure C.3: Measured input impedance data for the quadrifilar hemispherical helix, with the resistance (solid) and reactance (dashed) components shown.

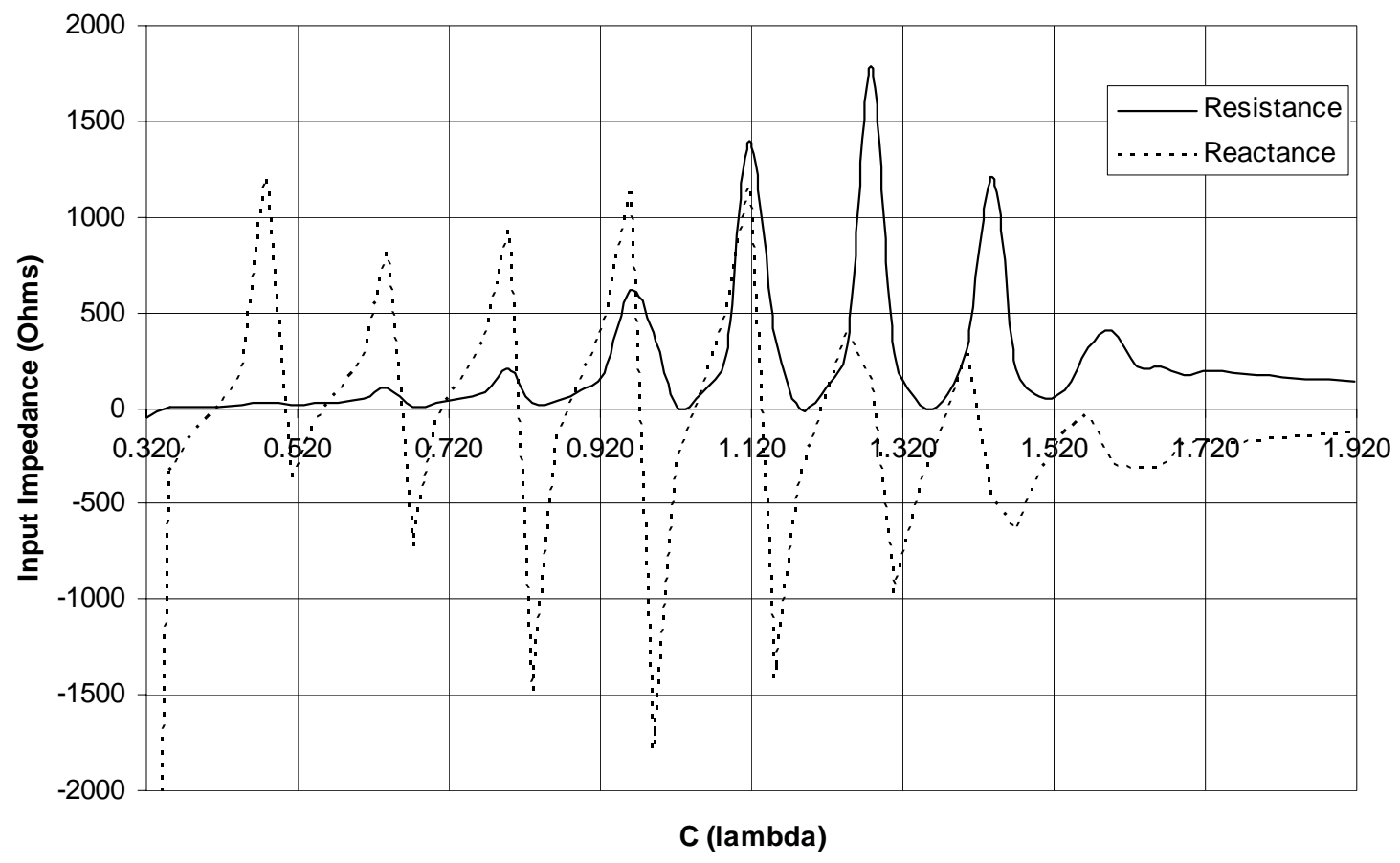

Figure C.4: Simulated input impedance data for the quadrifilar hemispherical helix, with the resistance (solid) and reactance (dashed) components shown. 


\section{Vita}

Jeffrey R. Clark was born on December 8, 1977 in Rochester, New York. He graduated Summa Cum Laude with a Bachelor of Science in physics from the University of Richmond in 2001. He is currently pursuing a Ph.D. in electrical engineering at Virginia Tech. 